\title{
A General and Selective Synthesis of Methylmonochlorosilanes from
}

\author{
Di-, Tri-, and Tetrachlorosilanes
}

\author{
Yuki Naganawa, ${ }^{*}$ Kei Sakamoto and Yumiko Nakajima* \\ *E-mail: yuki.naganawa@aist.go.jp/yumiko-nakajima@aist.go.jp \\ Interdisciplinary Research Center for Catalytic Chemistry (IRC3) \\ National Institute of Advanced Industrial Science and Technology (AIST) \\ 1-1-1 Higashi, Tsukuba, Ibaraki 305-8565, Japan
}

$\begin{array}{lr}\text { General Information } & \text { S2 } \\ \text { General Procedure } & \text { S3 } \\ \text { Supporting Table } & \text { S6 } \\ \text { Spectral Data } & \text { S12 } \\ \text { References } & \text { S25 } \\ { }^{1} \mathrm{H} \text { and }{ }^{13} \mathrm{C} \text { NMR Spectra } & \text { S26 }\end{array}$ 


\section{General Information}

All experiments were performed under argon atmosphere using Schlenk techniques or a glove box unless otherwise noted. 1,4-dioxane were purchased from Wako Pure Chemical Industries, Ltd. as "Super Dehydrated" and used as received. Dimethylaluminum chloride (ca. 1.0 M solution in hexane) was purchased from Kanto Chemical Co., Inc. Trimethylaluminium (ca. 1.4 M solution in hexane), triethylaluminum (1.0 M solution in hexane) and triisobutylaluminum (0.55 M solution in hexane) were purchased from Tokyo Chemical Industry Co., Ltd. Trioctylaluminium (25 wt\% solution in hexane) was purchased from Sigma-Aldrich, Inc. Other reagents were purchased from commercial suppliers and used without further purification. ${ }^{1} \mathrm{H}$, ${ }^{13} \mathrm{C}\left\{{ }^{1} \mathrm{H}\right\}$ and ${ }^{29} \mathrm{Si}\left\{{ }^{1} \mathrm{H}\right\}$ spectra were recorded on a Bruker AVANCE III HD 600 spectrometer. ${ }^{1} \mathrm{H},{ }^{13} \mathrm{C}\left\{{ }^{1} \mathrm{H}\right\}$ and ${ }^{29} \mathrm{Si}\left\{{ }^{1} \mathrm{H}\right\}$ NMR spectra $\left({ }^{1} \mathrm{H}, 600 \mathrm{MHz} ;{ }^{13} \mathrm{C}, 150 \mathrm{MHz}\right.$; ${ }^{29} \mathrm{Si}, 119 \mathrm{MHz}$ ) were recorded on a Bruker AVANCE 600 spectrometer. Chemical shifts are reported in $\delta(\mathrm{ppm})$ and referenced to the residual solvent signals for ${ }^{1} \mathrm{H}$ and ${ }^{13} \mathrm{C}$, and 1,4-bis(trimethylsilyl)benzene for ${ }^{29} \mathrm{Si}$ as external standards. High-resolution ESI mass spectra were obtained on a Bruker micrOTOF II. Column chromatography was performed with silica gel (Kanto Chemical Co., Inc. Silica gel 60 N, 100-210 $\mu \mathrm{m}$ ). 
Optimization of reaction conditions for methylation of $\mathrm{Me}_{2} \mathrm{SiCl}_{2}$ (1a) with methylaluminum reagents (Table 1)

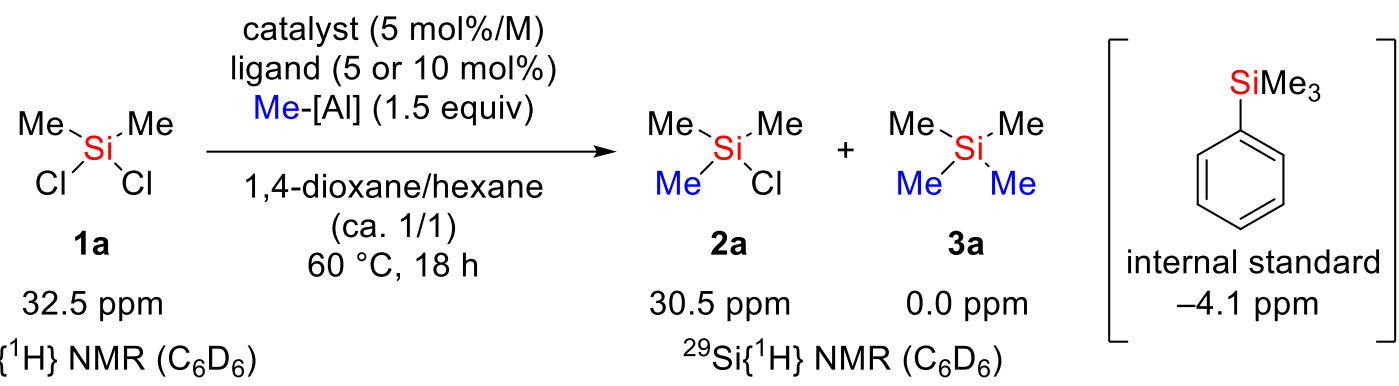

In a test tube with a PTFE screw cap, catalyst $(0.025 \mathrm{mmol} / \mathrm{M})$ and ligand $(0.025$ or $0.05 \mathrm{mmol})$ were equipped. 1,4-Dioxane $(0.5 \mathrm{~mL})$ was added into the test tube, and the solution was stirred at room temperature for ca. $5 \mathrm{~min}$. Next, dichlorodimethylsilane (1a) $(60 \mu \mathrm{L}, 0.5 \mathrm{mmol})$ and dimethylaluminum chloride $(750 \mu \mathrm{L}, 1.0 \mathrm{M}$ solution in hexane, $0.75 \mathrm{mmol}$ ) was successively added to the solution and the resulting mixture was stirred at $60{ }^{\circ} \mathrm{C}$ for $18 \mathrm{~h}$ in an oil bath. After cooling to room temperature, the conversion of $\mathbf{1 a}$ and the yield of chlorotrimethylsilane (2a) and tetramethylsilane (3a) was determined by ${ }^{29} \mathrm{Si}\left\{{ }^{1} \mathrm{H}\right\}$ NMR spectroscopic analysis with trimethyl(phenyl)silane (86 $\mu 1,0.5 \mathrm{mmol})$ as internal standard.

The reactions of trichloromethylsilane (5a) (Table 4) and tetrachlorosilane (6) (Scheme 3) were conducted with the similar procedure.

${ }^{29} \mathrm{Si}\left\{{ }^{1} \mathrm{H}\right\}$ NMR (119 MHz, $\left.\mathrm{CDCl}_{3}\right): \delta-18.4 \mathrm{ppm}\left(\mathrm{SiCl}_{4}\right), 13.1 \mathrm{ppm}\left(\mathrm{MeSiCl}_{3}\right), 32.5$ ppm $\left(\mathrm{Me}_{2} \mathrm{SiCl}_{2}\right), 30.5 \mathrm{ppm}\left(\mathrm{Me}_{3} \mathrm{SiCl}\right), 0.0 \mathrm{ppm}\left(\mathrm{Me}_{4} \mathrm{Si}\right),-4.1 \mathrm{ppm}\left(\mathrm{Me}_{3} \mathrm{SiPh}\right.$; internal standard)

General procedure for palladium-catalyzed methylation of dichlorosilane (1) with dimethylaluminum chloride (Table 2)

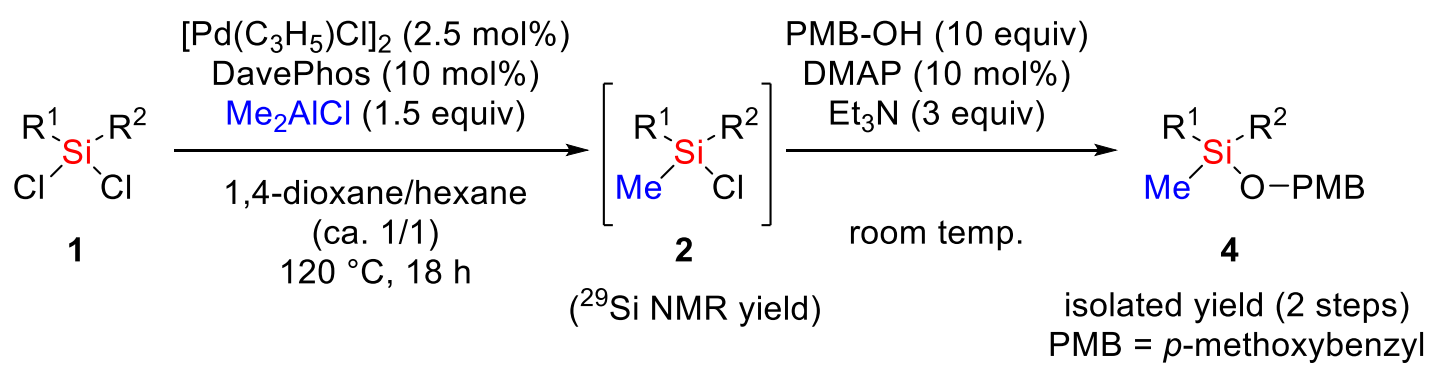

In a test tube with a PTFE screw cap, $[\mathrm{Pd}(\text { allyl }) \mathrm{Cl}]_{2}(4.6 \mathrm{mg}, 0.0125 \mathrm{mmol})$ and DavePhos (19.7 mg, $0.05 \mathrm{mmol})$ were equipped. 1,4-Dioxane $(0.5 \mathrm{~mL})$ was added into 
the test tube, and the solution was stirred at room temperature for ca. 5 min. Next, dichlorosilanes $1(0.5 \mathrm{mmol})$ and dimethylaluminum chloride $(750 \mu \mathrm{L}, 1.0 \mathrm{M}$ solution in hexane, $0.75 \mathrm{mmol}$ ) was successively added to the solution and the resulting mixture was stirred at $120{ }^{\circ} \mathrm{C}$ for $18 \mathrm{~h}$ in an oil bath. After cooling to room temperature, the yield of 2 was determined by ${ }^{29} \mathrm{Si}\left\{{ }^{1} \mathrm{H}\right\}$ NMR spectroscopic analysis with trimethyl(phenyl)silane $(86 \mu \mathrm{l}, \quad 0.5 \mathrm{mmol})$ as internal standard. A series of methylmonochlorosilane $\mathbf{2}$ was identified by analogy with the trimethylchlorosilane (2a) as summarized in Table S1. After the determination of NMR yield of 2, all the reaction mixture was treated with $\mathrm{Et}_{3} \mathrm{~N}(209 \mu \mathrm{L}, 1.5 \mathrm{mmol})$, DMAP $(6.1 \mathrm{mg}, 0.05$ mmol) and 4-methoxybenzyl alcohol $(622 \mu \mathrm{L}, 5.0 \mathrm{mmol})$, and the resulting mixture was stirred at room temperature for $1 \mathrm{~h}$. Then, the mixture was diluted with hexane, filtered through a pad of Celite and the filtrate was concentrated to dryness under vacuum. The residue was purified by column chromatography on silica gel with hexane/AcOEt (40/1 to 30/1) to afford the corresponding methylalkoxysilanes 4.

The reactions of trialkylaluminum (Table S2) were conducted with the similar procedure. A series of alkylmonochlorosilane 2 was identified by analogy with the trimethylchlorosilane (2a) as summarized in Table S3.

\section{Procedure for a gram-scale synthesis of $2 p$ (Scheme 2).}

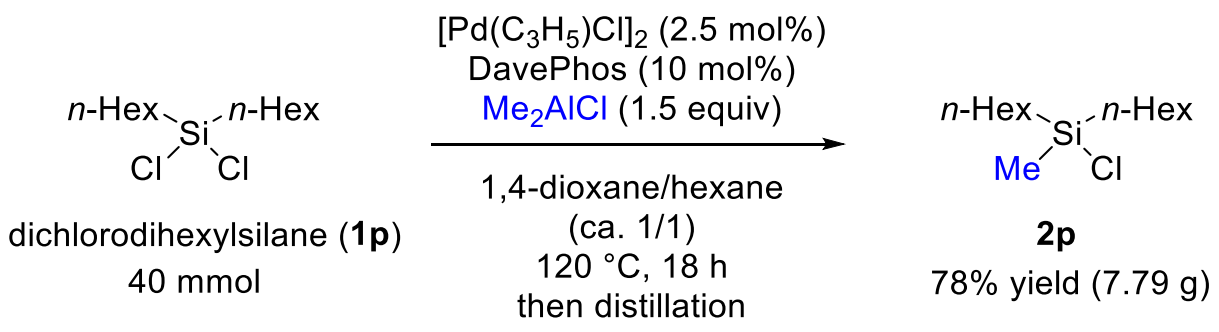

In a Schlenk flask, $[\mathrm{Pd}(\text { allyl }) \mathrm{Cl}]_{2}(366 \mathrm{mg}, 1.0 \mathrm{mmol})$ and DavePhos $(1574 \mathrm{mg}, 4.0$ mmol) were equipped under argon. 1,4-Dioxane $(60 \mathrm{~mL})$ was added and the solution was stirred at room temperature for ca. $5 \mathrm{~min}$. Next, dichlorodihexylsilane $(11.1 \mathrm{~mL}, 40$ $\mathrm{mmol})$ and dimethylaluminum chloride $(60 \mathrm{~mL}, 1.0 \mathrm{M}$ solution in hexane, $60 \mathrm{mmol})$ was successively added to the solution and the resulting mixture was stirred at $120{ }^{\circ} \mathrm{C}$ for $18 \mathrm{~h}$ in an oil bath. After the reaction, the mixture was diluted with hexane, filtered through a pad of Celite under argon and the filtrate was concentrated to dryness under vacuum. The resulting crude mixture was purified by distillation $\left(90^{\circ} \mathrm{C}, 0.2\right.$ Torr $)$ to give $2 \mathbf{p}$ in $78 \%$ yield $(7.79 \mathrm{~g})$. 
${ }^{1} \mathrm{H}$ NMR (600 MHz, $\left.\mathrm{C}_{6} \mathrm{D}_{6}\right): \delta 1.39$ (m, 4H, hexyl), 1.35-1.18 (m, 12H, hexyl), 0.89 (t, $J$ $\left.=7.2 \mathrm{~Hz}, 6 \mathrm{H}, \mathrm{CH}_{2} \underline{\mathrm{C}}_{3}\right), 0.70\left(\mathrm{~m}, 4 \mathrm{H}, \mathrm{SiC}_{2}\right), 0.26$ (s, 3H, $\left.\mathrm{SiC}_{3}\right)$.

${ }^{13} \mathrm{C}\left\{{ }^{1} \mathrm{H}\right\}$ NMR $\left(150 \mathrm{MHz}, \mathrm{C}_{6} \mathrm{D}_{6}\right): \delta 33.1,31.8,23.3,22.9,17.9,14.3,-0.1$.

${ }^{29} \mathrm{Si}\left\{{ }^{1} \mathrm{H}\right\}$ NMR (119 MHz, $\left.\mathrm{C}_{6} \mathrm{D}_{6}\right): \delta 32.4$.

The spectrum data of $\mathbf{2 p}$ was in accordance with the literature. ${ }^{[\mathrm{S} 1]}$

\section{General procedure for palladium-catalyzed methylation of trichlorosilane (5) with dimethylaluminum chloride (Table 4)}

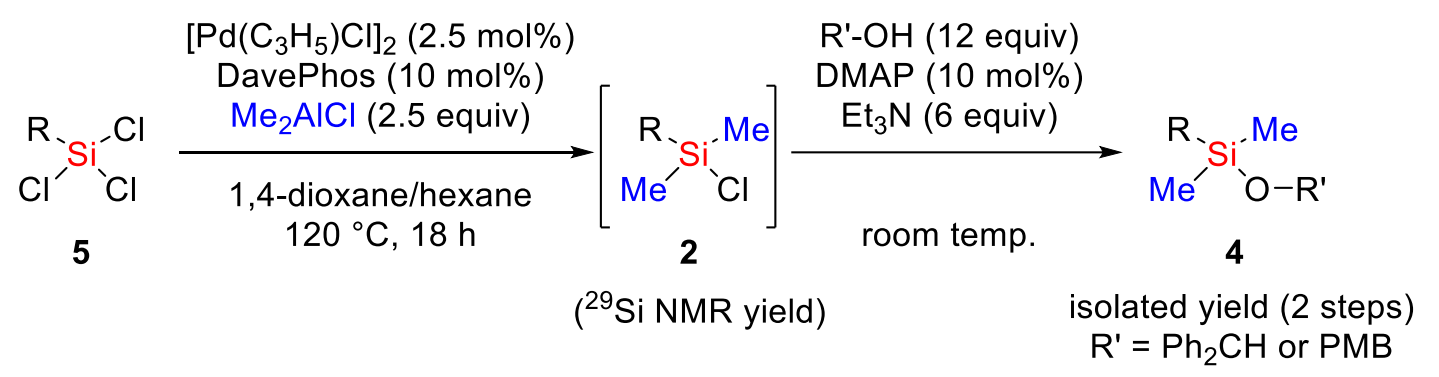

In a test tube with a PTFE screw cap, $[\mathrm{Pd}(\text { allyl }) \mathrm{Cl}]_{2}(4.6 \mathrm{mg}, 0.0125 \mathrm{mmol})$ and DavePhos (19.7 mg, $0.05 \mathrm{mmol})$ were equipped. 1,4-Dioxane (1.0 mL) was added into the test tube, and the solution was stirred at room temperature for ca. 5 min. Next, trichlorosilanes $5(0.5 \mathrm{mmol})$ and dimethylaluminum chloride $(1.25 \mathrm{~mL}, 1.0 \mathrm{M}$ solution in hexane, $1.25 \mathrm{mmol}$ ) was successively added to the solution and the resulting mixture was stirred at $120{ }^{\circ} \mathrm{C}$ for $18 \mathrm{~h}$ in an oil bath. After cooling to room temperature, yield of 2 was determined by ${ }^{29} \mathrm{Si}\left\{{ }^{1} \mathrm{H}\right\} \quad$ NMR spectroscopic analysis with trimethyl(phenyl)silane $(86 \mu 1,0.5 \mathrm{mmol})$ as internal standard. A series of dimethylmonochlorosilane 2 was identified by analogy with the trimethylchlorosilane (2a) as summarized in Table S4. After the determination of NMR yield of 2, all the reaction mixture was treated with $\mathrm{Et}_{3} \mathrm{~N}(417 \mu \mathrm{L}, 3.0 \mathrm{mmol})$, DMAP $(6.1 \mathrm{mg}, 0.05$ $\mathrm{mmol})$ and 4-methoxybenzyl alcohol $(747 \mu \mathrm{L}, 6.0 \mathrm{mmol})$, and the resulting mixture was stirred at room temperature for $1 \mathrm{~h}$. Then, the mixture was diluted with hexane, filtered through a pad of Celite and the filtrate was concentrated to dryness under vacuum. The residue was purified by column chromatography on silica gel with hexane/AcOEt (40/1 to 30/1) to afford the corresponding alkylsilanes 4 .

The reactions of trialkylaluminum (Table S5) were conducted with the similar procedure. A series of alkylmonochlorosilane $\mathbf{2}$ was identified by analogy with the trimethylchlorosilane (2a) as summarized in Table $\mathbf{S 6 .}$ 


\section{Supporting Table}

Table S1. ${ }^{29} \mathrm{Si}\left\{{ }^{1} \mathrm{H}\right\}$ NMR spectroscopic data $\left(\mathrm{C}_{6} \mathrm{D}_{6}\right)$ for Table 2.

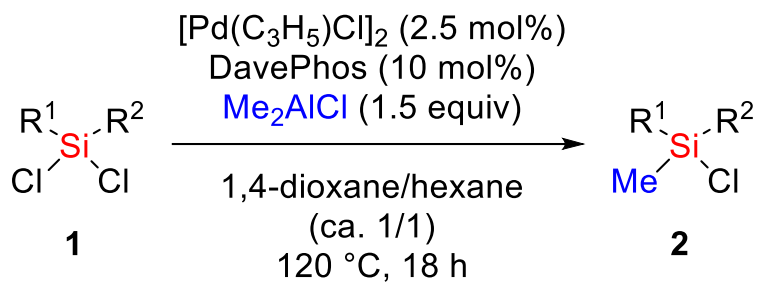

\begin{tabular}{|c|c|c|c|}
\hline $\mathrm{R}^{1}$ & $\mathrm{R}^{2}$ & $\delta(\mathrm{ppm})(\mathbf{1})^{\mathrm{a}}$ & $\delta(\mathrm{ppm})(\mathbf{2})^{\mathrm{b}}$ \\
\hline$n$-propyl & $\mathrm{Me}$ & $33.0(1 \mathrm{~b})$ & $31.0(\mathbf{2 b})$ \\
\hline$n$-hexyl & $\mathrm{Me}$ & $33.5(1 \mathrm{c})$ & $31.3(\mathbf{2 c})$ \\
\hline$n$-decyl & $\mathrm{Me}$ & $33.4(\mathbf{1 d})$ & $31.3(\mathbf{2 d})$ \\
\hline$n$-dodecyl & $\mathrm{Me}$ & $33.4(1 \mathrm{e})$ & $31.2(\mathbf{2 e})$ \\
\hline$n$-octadecyl & $\mathrm{Me}$ & 33.4 (1f) & $31.2(\mathbf{2 f})$ \\
\hline phenethyl & $\mathrm{Me}$ & $32.8(\mathbf{1 g})$ & $31.2(\mathbf{2 g})$ \\
\hline 3-chloropropyl & $\mathrm{Me}$ & $32.6(\mathbf{l h})$ & $31.3(\mathbf{2 h})$ \\
\hline 3,3,3-trifluoropropyl & $\mathrm{Me}$ & 31.6 (1i) & $30.6(\mathbf{2 i})$ \\
\hline cyclohexyl & $\mathrm{Me}$ & $33.0(\mathbf{1} \mathbf{j})$ & $31.3(\mathbf{2} \mathbf{j})$ \\
\hline phenyl & $\mathrm{Me}$ & $19.3(\mathbf{1 k})$ & $20.2(\mathbf{2 k})^{\mathrm{a}}$ \\
\hline phenyethenyl & $\mathrm{Me}$ & $18.3(1 \mathrm{l})^{\mathrm{c}}$ & $18.9(2 \mathrm{l})$ \\
\hline ethyl & ethyl & 36.5 (1m) & $34.5(2 \mathrm{~m})$ \\
\hline$n$-butyl & $n$-butyl & $34.1(\mathbf{1 n})$ & $32.0(\mathbf{2 n})$ \\
\hline$n$-pentyl & $n$-pentyl & $34.0(10)$ & $31.9(\mathbf{2 0})$ \\
\hline$n$-hexyl & $n$-hexyl & $34.0(1 \mathbf{p})$ & $31.9(\mathbf{2 p})$ \\
\hline$n$-octyl & $n$-octyl & $34.0(1 \mathrm{q})$ & $31.9(\mathbf{2 q})$ \\
\hline 2-ethylhexyl & 2-ethylhexyl & $33.9(\mathbf{1 r})$ & $31.7(\mathbf{2 r})$ \\
\hline isopropyl & isopropyl & $38.5(1 \mathrm{~s})$ & $36.9(\mathbf{2 s})$ \\
\hline
\end{tabular}

${ }^{a}$ Identified with a commercially available compound as reference ${ }^{b}$ Identified by analogy with the trimethylchlorosilane (2a) ${ }^{\mathrm{c}}$ Identified with a compound synthesized via silyl-Heck reaction ${ }^{[\mathrm{S} 2]}$ as reference. 
Table S2. Scope of palladium-catalyzed monoalkyation of $\mathbf{1 a}$ with trialkylaluminum. ${ }^{\mathrm{a}}$

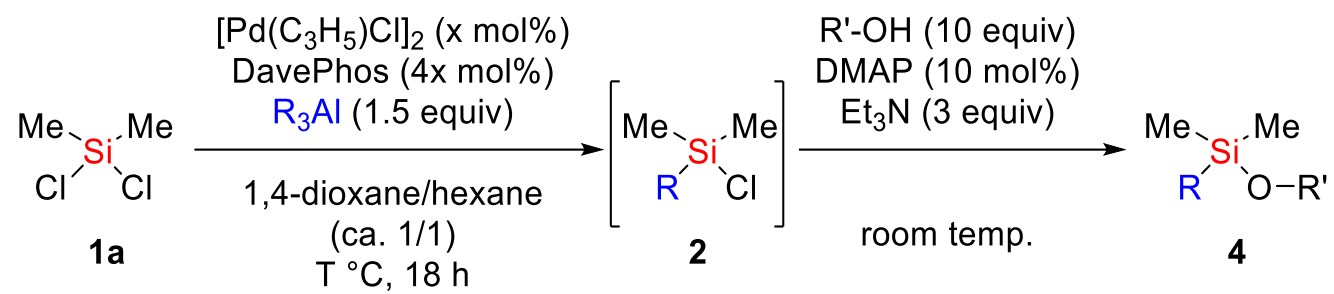

\begin{tabular}{cccccccc}
\hline entry & $\begin{array}{c}\mathrm{x} \\
(\mathrm{mol} \%)\end{array}$ & $\mathrm{R}^{\mathrm{b}}$ & $\begin{array}{c}\mathrm{T} \\
\left({ }^{\circ} \mathrm{C}\right)\end{array}$ & $\begin{array}{c}\text { \% conv. } \\
(\mathbf{1 a})^{\mathrm{c}}\end{array}$ & $\begin{array}{c}\text { \% yield } \\
(\mathbf{2})^{\mathrm{c}}\end{array}$ & $\mathrm{R}^{\prime}$ & $\begin{array}{c}\% \text { yield } \\
(\mathbf{4})^{\mathrm{d}}\end{array}$ \\
\hline 1 & 2.5 & $\mathrm{Et}$ & 60 & $>99$ & $95(\mathbf{2 u})$ & $\mathrm{Ph}_{2} \mathrm{CH}$ & $92(\mathbf{4 u})$ \\
2 & 5 & $n$-Bu $^{\mathrm{d}}$ & 120 & $>99$ & $95(\mathbf{2 a a})$ & $\mathrm{PMB}$ & $90(\mathbf{4 a a})$ \\
3 & 5 & $n$-octyl & 120 & $>99$ & $88(\mathbf{2 v})$ & $\mathrm{PMB}$ & $84(\mathbf{4 v})$ \\
4 & 5 & $i$-Bu & 180 & $>99$ & $47(\mathbf{2 x})$ & $\mathrm{PMB}$ & $45(\mathbf{4 x})$ \\
\hline
\end{tabular}

${ }^{a}$ Reaction conditions: $\left[\mathrm{Pd}\left(\mathrm{C}_{3} \mathrm{H}_{5}\right) \mathrm{Cl}\right]_{2}(2.5$ or $5.0 \mathrm{~mol} \%)$, DavePhos (10 or $\left.20 \mathrm{~mol} \%\right), 1$ (0.5 mmol), $\mathrm{R}_{3} \mathrm{Al}(0.75 \mathrm{mmol})$ in dioxane $(1.5 \mathrm{~mL}), 60,120$ or $180{ }^{\circ} \mathrm{C}, 18 \mathrm{~h}$; triethylamine $\quad(1.5 \mathrm{mmol}), \quad$ 4-dimethylaminopyridine $\quad(10 \quad \mathrm{~mol} \%)$, p-methoxybenzylalcohol or diphenylmethanol (for $\mathbf{2 u}$ ) $(5 \mathrm{mmol})$, r.t., $3 \mathrm{~h}$. b Concentration of methylaluminum reagents: $\mathrm{Et}_{3} \mathrm{Al}\left(1.0 \mathrm{M}\right.$ in hexane), (n-octyl) ${ }_{3} \mathrm{Al}$ $\left(25 \mathrm{wt} \%\right.$ in hexane), $(i \mathrm{Bu})_{3} \mathrm{Al}\left(0.55 \mathrm{M}\right.$ in hexane). ${ }^{\mathrm{c}}$ Determined by ${ }^{29} \mathrm{Si}\left\{{ }^{1} \mathrm{H}\right\} \mathrm{NMR}$ spectroscopic analysis with trimethyl(phenyl)silane $(0.5 \mathrm{mmol})$ as an internal standard. ${ }^{\mathrm{d}}$ Isolated yield (2 steps). ${ }^{\mathrm{d}}$ Prepared from $\mathrm{AlCl}_{3}(0.75 \mathrm{mmol})$ and $n$-BuLi (3 equiv) in hexane ( $2 \mathrm{~mL})$, r.t., $3 \mathrm{~h}$. 
Table S3. ${ }^{29} \mathrm{Si}\left\{{ }^{1} \mathrm{H}\right\}$ NMR spectroscopic data $\left(\mathrm{C}_{6} \mathrm{D}_{6}\right)$ for Table $\mathrm{S} 2$.

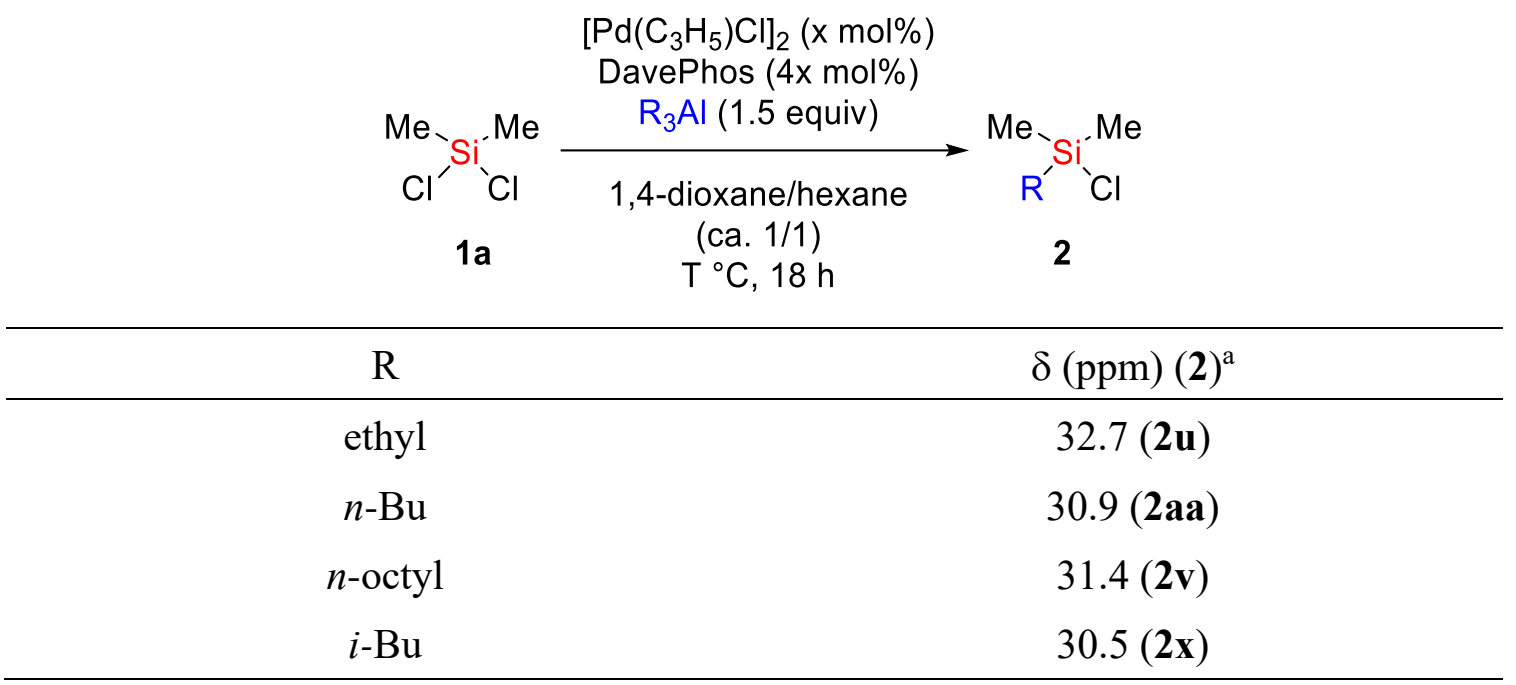

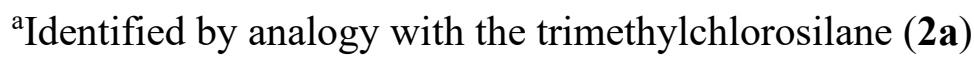


Table S4. ${ }^{29} \mathrm{Si}\left\{{ }^{1} \mathrm{H}\right\}$ NMR spectroscopic data $\left(\mathrm{C}_{6} \mathrm{D}_{6}\right)$ for Table 4.

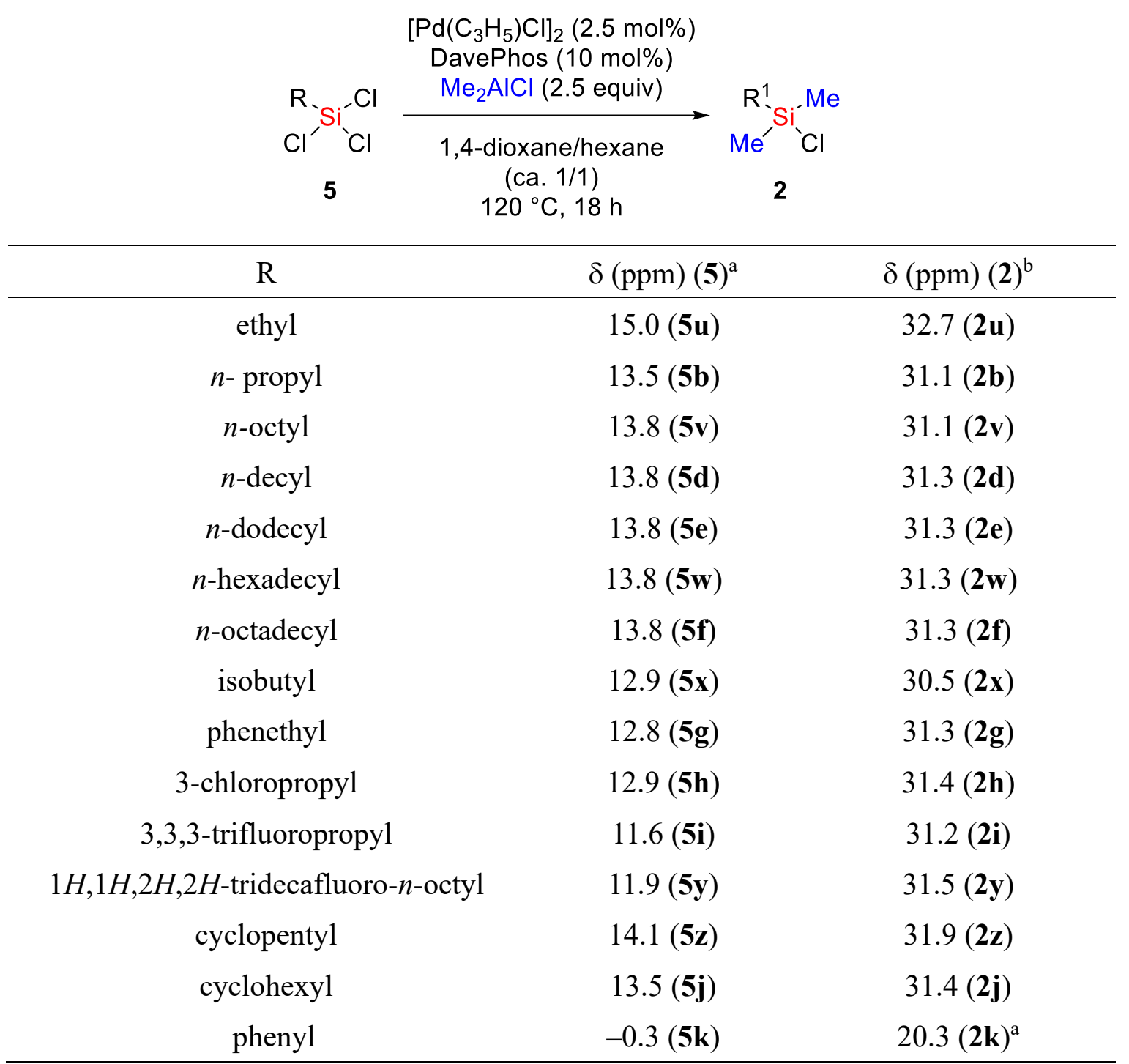

${ }^{a}$ Identified with a commercially available compound as reference ${ }^{b}$ Identified by analogy with the trimethylchlorosilane (2a) 
Table S5. Scope of palladium-catalyzed double alkylation of 5a with trialkylaluminum. ${ }^{\text {a }}$

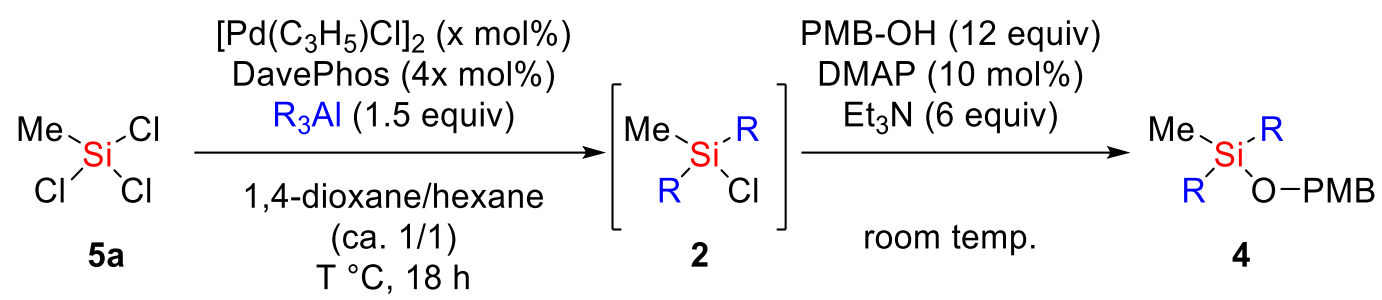

\begin{tabular}{ccccccc}
\hline entry & $\begin{array}{c}\mathrm{x} \\
(\mathrm{mol} \%)\end{array}$ & $\mathrm{R}^{\mathrm{b}}$ & $\mathrm{T}\left({ }^{\circ} \mathrm{C}\right)$ & $\begin{array}{c}\text { \% conv. } \\
(\mathbf{5 a})^{\mathrm{c}}\end{array}$ & $\begin{array}{c}\text { \% yield } \\
(\mathbf{2})^{\mathrm{c}}\end{array}$ & $\begin{array}{c}\text { \% yield } \\
(\mathbf{4})^{\mathrm{d}}\end{array}$ \\
\hline 1 & 2.5 & Et & 120 & $>99$ & $86(\mathbf{2 m})$ & $68(\mathbf{4 m})$ \\
2 & 5 & $n$-octyl & 180 & $>99$ & $62(\mathbf{2 q})$ & $61(\mathbf{4 q})$ \\
3 & 5 & $i \mathrm{Bu}$ & 180 & 70 & $<5^{\mathrm{e}}$ & - \\
\hline
\end{tabular}

${ }^{a}$ Reaction conditions: $\left[\mathrm{Pd}\left(\mathrm{C}_{3} \mathrm{H}_{5}\right) \mathrm{Cl}\right]_{2}(2.5$ or $5.0 \mathrm{~mol} \%)$, DavePhos (10 or $\left.20 \mathrm{~mol} \%\right), 1$ (0.5 mmol), $\mathrm{R}_{3} \mathrm{Al}(0.75 \mathrm{mmol})$ in dioxane $(1.5 \mathrm{~mL}), 60$ or $120{ }^{\circ} \mathrm{C}, 18 \mathrm{~h}$; then triethylamine (3 mmol), 4-dimethylaminopyridine (10 mol\%), $p$-methoxybenzylalcohol (6 mmol), r.t., 3 h. ${ }^{b}$ Concentration of methylaluminum reagents: Et ${ }_{3} \mathrm{Al}$ (1.0 $\mathrm{M}$ in hexane), ( $n$-octyl) $)_{3} \mathrm{Al}\left(25 \mathrm{wt} \%\right.$ in hexane), $(i \mathrm{Bu})_{3} \mathrm{Al}\left(0.55 \mathrm{M}\right.$ in hexane). ${ }^{\mathrm{c}}$ Determined by ${ }^{29} \mathrm{Si}\left\{{ }^{1} \mathrm{H}\right\}$ NMR spectroscopic analysis with trimethyl(phenyl)silane $(0.5 \mathrm{mmol})$ as an internal standard. ${ }^{\mathrm{d}}$ Isolated yield (2 steps). ${ }^{\mathrm{e}} i \mathrm{BuMeSiCl}_{2}$ was observed in $39 \% \mathrm{NMR}$ yield. 
Table S6. ${ }^{29} \mathrm{Si}\left\{{ }^{1} \mathrm{H}\right\}$ NMR spectroscopic data $\left(\mathrm{C}_{6} \mathrm{D}_{6}\right)$ for Table $\mathrm{S} 5$.

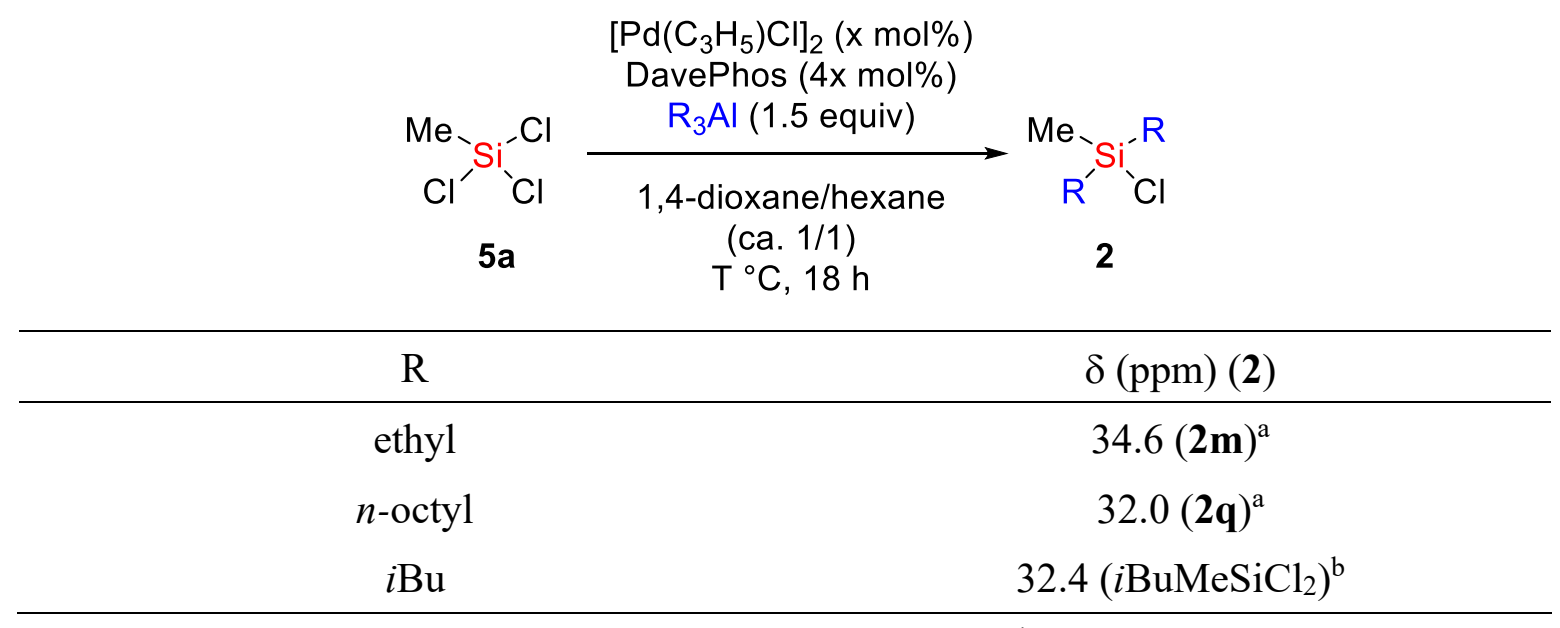

${ }^{a}$ Identified by analogy with the trimethylchlorosilane (2a) ${ }^{b}$ Identified by analogy with the dimethyldichlorosilane (1a) and ${ }^{1} \mathrm{H}$ NMR analysis 


\section{Spectral Data}

(4-methoxybenzyloxy)dimethyl(n-propyl)silane (4b)

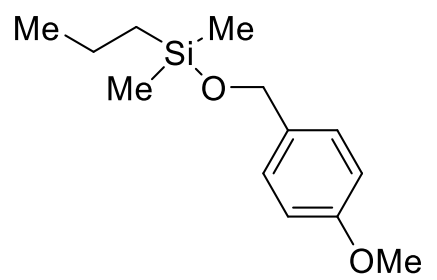

Purified by silica gel chromatography (eluent; hexane/AcOEt $=30 / 1$ ), obtained as colorless oil (87\% yield, $103.6 \mathrm{mg})$.

${ }^{1} \mathrm{H}$ NMR (600 MHz, $\left.\mathrm{CDCl}_{3}\right): \delta 7.24(\mathrm{~d}, J=8.6 \mathrm{~Hz}, 2 \mathrm{H}, \mathrm{Ar}), 6.87(\mathrm{~d}, J=8.6 \mathrm{~Hz}, 2 \mathrm{H}, \mathrm{Ar})$, $4.63\left(\mathrm{~s}, 2 \mathrm{H}, \mathrm{OC}_{2} \mathrm{Ar}\right), 3.80\left(\mathrm{~s}, 3 \mathrm{H}, \mathrm{OC}_{3}\right), 1.39\left(\mathrm{~m}, 2 \mathrm{H}, \mathrm{CH}_{2} \underline{\mathrm{C}}_{2} \mathrm{CH}_{3}\right), 0.96$ (t, $J=7.3$

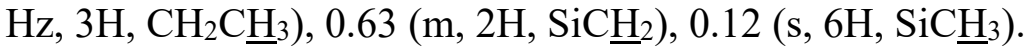

${ }^{13} \mathrm{C}\left\{{ }^{1} \mathrm{H}\right\}$ NMR $\left(150 \mathrm{MHz}, \mathrm{CDCl}_{3}\right): \delta 158.8,133.2,128.0,113.7,64.4,55.3,19.0,18.1$, $16.7,-1.9$.

${ }^{29} \mathrm{Si}\left\{{ }^{1} \mathrm{H}\right\}$ NMR $\left(119 \mathrm{MHz}, \mathrm{CDCl}_{3}\right): \delta$ 18.6.

HRMS (ESI) m/z: [M + Na ${ }^{+}$Calcd for $\mathrm{C}_{13} \mathrm{H}_{22} \mathrm{O}_{2} \mathrm{SiNa}$ : 261.1281; Found: 261.1291.

\section{(4-methoxybenzyloxy)dimethyl( $n$-hexyl)silane (4c)}

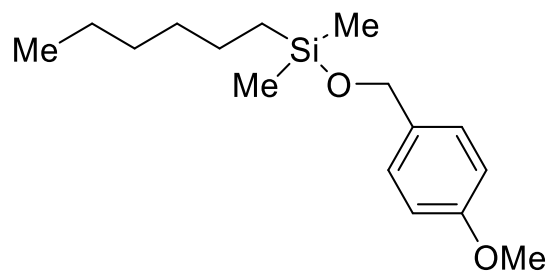

Purified by silica gel chromatography (eluent; hexane/AcOEt $=30 / 1$ ), obtained as colorless oil (91\% yield, $127.8 \mathrm{mg}$ ).

${ }^{1} \mathrm{H}$ NMR (600 MHz, $\left.\mathrm{CDCl}_{3}\right): \delta 7.24(\mathrm{~d}, J=8.6 \mathrm{~Hz}, 2 \mathrm{H}, \mathrm{Ar}), 6.87(\mathrm{~d}, J=8.6 \mathrm{~Hz}, 2 \mathrm{H}, \mathrm{Ar})$, $4.63\left(\mathrm{~s}, 2 \mathrm{H}, \mathrm{OC}_{2} \mathrm{Ar}\right), 3.80\left(\mathrm{~s}, 3 \mathrm{H}, \mathrm{OCH}_{3}\right), 1.40-1.20(\mathrm{~m}, 8 \mathrm{H}$, hexyl), $0.88(\mathrm{t}, J=7.2 \mathrm{~Hz}$,

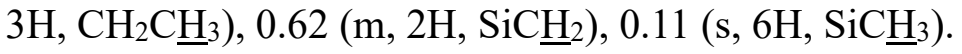

${ }^{13} \mathrm{C}\left\{{ }^{1} \mathrm{H}\right\} \mathrm{NMR}\left(150 \mathrm{MHz}, \mathrm{CDCl}_{3}\right): \delta 158.8,133.2,128.0,113.7,64.4,55.3,33.1,31.6$, $23.1,22.6,16.4,14.1,-2.0$.

${ }^{29} \mathrm{Si}\left\{{ }^{1} \mathrm{H}\right\}$ NMR $\left(119 \mathrm{MHz}, \mathrm{CDCl}_{3}\right): \delta 18.8$.

HRMS (ESI) m/z: [M + Na] $]^{+}$Calcd for $\mathrm{C}_{16} \mathrm{H}_{28} \mathrm{O}_{2} \mathrm{SiNa}$ : 303.1751; Found: 303.1756 .

(4-methoxybenzyloxy)dimethyl(n-decyl)silane (4d) 


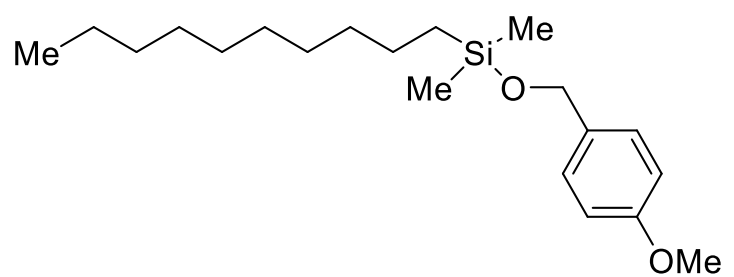

Purified by silica gel chromatography (eluent; hexane/AcOEt $=30 / 1$ ), obtained as colorless oil ( $89 \%$ yield, $150.0 \mathrm{mg})$.

${ }^{1} \mathrm{H}$ NMR (600 MHz, $\left.\mathrm{CDCl}_{3}\right): \delta 7.24(\mathrm{~d}, J=8.7 \mathrm{~Hz}, 2 \mathrm{H}, \mathrm{Ar}), 6.87(\mathrm{~d}, J=8.7 \mathrm{~Hz}, 2 \mathrm{H}, \mathrm{Ar})$, $4.63\left(\mathrm{~s}, 2 \mathrm{H}, \mathrm{OC}_{2} \mathrm{Ar}\right), 3.80\left(\mathrm{~s}, 3 \mathrm{H}, \mathrm{OCH}_{3}\right), 1.40-1.20(\mathrm{~m}, 16 \mathrm{H}$, decyl), $0.88(\mathrm{t}, J=7.2$ $\left.\mathrm{Hz}, 3 \mathrm{H}, \mathrm{CH}_{2} \mathrm{C}_{3}\right), 0.61\left(\mathrm{~m}, 2 \mathrm{H}, \mathrm{SiCH}_{2}\right), 0.11$ (s, 6H, SiC$\left.\underline{H}_{3}\right)$.

${ }^{13} \mathrm{C}\left\{{ }^{1} \mathrm{H}\right\} \mathrm{NMR}\left(150 \mathrm{MHz}, \mathrm{CDCl}_{3}\right): \delta 158.8,133.3,128.1,113.7,64.4,55.3,33.5,32.0$, 29.7, 29.6, 29.4 (2C were overlapped.), 23.2, 22.7, 16.4, 14.2, -2.0.

${ }^{29} \mathrm{Si}\left\{{ }^{1} \mathrm{H}\right\} \mathrm{NMR}\left(119 \mathrm{MHz}, \mathrm{CDCl}_{3}\right): \delta 18.9$.

HRMS (ESI) m/z: $[\mathrm{M}+\mathrm{Na}]^{+}$Calcd for $\mathrm{C}_{20} \mathrm{H}_{36} \mathrm{O}_{2} \mathrm{SiNa}$ : 359.2377; Found: 359.2374 .

\section{(4-methoxybenzyloxy)dimethyl(n-dodecyl)silane (4e)}

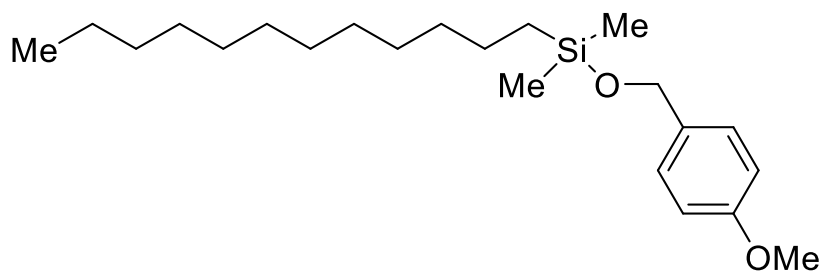

Purified by silica gel chromatography (eluent; hexane/AcOEt $=40 / 1$ ), obtained as colorless oil ( $89 \%$ yield, $162.8 \mathrm{mg}$ ).

${ }^{1} \mathrm{H}$ NMR (600 MHz, $\left.\mathrm{CDCl}_{3}\right): \delta 7.24(\mathrm{~d}, J=8.6 \mathrm{~Hz}, 2 \mathrm{H}, \mathrm{Ar}), 6.86(\mathrm{~d}, J=8.6 \mathrm{~Hz}, 2 \mathrm{H}, \mathrm{Ar})$,

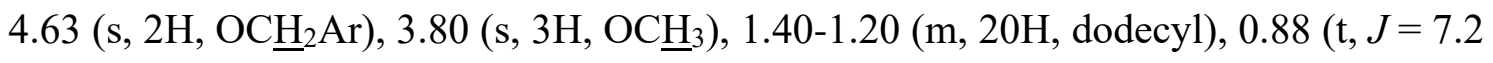

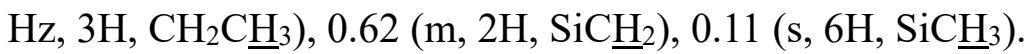

${ }^{13} \mathrm{C}\left\{{ }^{1} \mathrm{H}\right\}$ NMR $\left(150 \mathrm{MHz}, \mathrm{CDCl}_{3}\right): \delta 158.8,133.3,128.1,113.7,64.4,55.3,33.5,32.0$, 29.74, 29.72, 29.68, 29.62, 29.4 (2C were overlapped.), 23.2, 22.7, 16.4, 14.2, -2.0. ${ }^{29} \mathrm{Si}\left\{{ }^{1} \mathrm{H}\right\} \mathrm{NMR}\left(119 \mathrm{MHz}, \mathrm{CDCl}_{3}\right): \delta 18.9$.

HRMS (ESI) m/z: [M + Na $]^{+}$Calcd for $\mathrm{C}_{22} \mathrm{H}_{40} \mathrm{O}_{2} \mathrm{SiNa}$ : 387.2690; Found: 387.2685. 


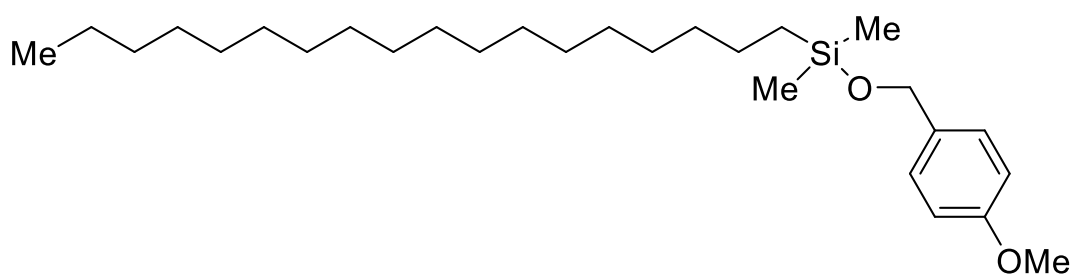

Purified by silica gel chromatography (eluent; hexane/AcOEt $=30 / 1$ ), obtained as colorless oil (91\% yield, $205.3 \mathrm{mg})$.

${ }^{1} \mathrm{H}$ NMR (600 MHz, $\left.\mathrm{CDCl}_{3}\right): \delta 7.24(\mathrm{~d}, J=8.6 \mathrm{~Hz}, 2 \mathrm{H}, \mathrm{Ar}), 6.87$ (d, $\left.J=8.6 \mathrm{~Hz}, 2 \mathrm{H}, \mathrm{Ar}\right)$, $4.63\left(\mathrm{~s}, 2 \mathrm{H}, \mathrm{OC}_{2} \mathrm{Ar}\right), 3.80\left(\mathrm{~s}, 3 \mathrm{H}, \mathrm{OC}_{3}\right), 1.40-1.20(\mathrm{~m}, 32 \mathrm{H}$, octadecyl), $0.88(\mathrm{t}, J=$ $\left.7.2 \mathrm{~Hz}, 3 \mathrm{H}, \mathrm{CH}_{2} \mathrm{CH}_{3}\right), 0.61$ (m, $\left.2 \mathrm{H}, \mathrm{SiC}_{2}\right), 0.11$ (s, $\left.6 \mathrm{H}, \mathrm{SiC}_{3}\right)$.

${ }^{13} \mathrm{C}\left\{{ }^{1} \mathrm{H}\right\}$ NMR $\left(150 \mathrm{MHz}, \mathrm{CDCl}_{3}\right): \delta 158.8,133.3,128.1,113.7,64.4,55.3,33.5,32.0$. 29.73 (8C were overlapped.), 29.69, 29.62, 29.4 (2C were overlapped.), 23.2, 22.7, 16.4, $14.2,-2.0$.

${ }^{29} \mathrm{Si}\left\{{ }^{1} \mathrm{H}\right\} \mathrm{NMR}\left(119 \mathrm{MHz}, \mathrm{CDCl}_{3}\right): \delta 18.9$.

HRMS (ESI) m/z: $[\mathrm{M}+\mathrm{Na}]^{+}$Calcd for $\mathrm{C}_{28} \mathrm{H}_{52} \mathrm{O}_{2} \mathrm{SiNa}$ : 471.3629; Found: 471.3631.

\section{(4-methoxybenzyloxy)dimethyl(phenethyl)silane (4g)}

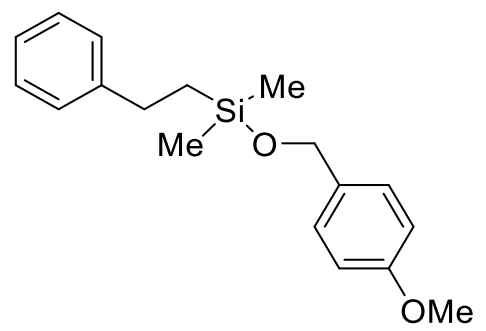

Purified by silica gel chromatography (eluent; hexane/AcOEt $=30 / 1)$, obtained as colorless oil ( $85 \%$ yield, $127.4 \mathrm{mg}$ ).

${ }^{1} \mathrm{H}$ NMR (600 MHz, $\mathrm{CDCl}_{3}$ ): $\delta$ 7.30-7.22 (m, 4H, Ar), 7.21-7.13 (m, 3H, Ar), 6.88 (m,

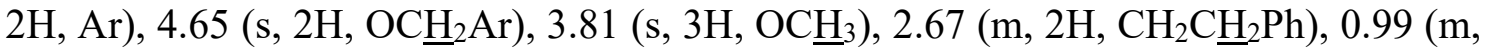
$\left.2 \mathrm{H}, \mathrm{SiC}_{2}\right), 0.15$ (s, 6H, $\left.\mathrm{SiC}_{3}\right)$.

${ }^{13} \mathrm{C}\left\{{ }^{1} \mathrm{H}\right\}$ NMR $\left(150 \mathrm{MHz}, \mathrm{CDCl}_{3}\right): \delta 158.9,144.9,133,1$ 128.3, 128.1, 127.8, 125.6, $113.8,64.5,55.3,29.3,18.5,-2.0$.

${ }^{29} \mathrm{Si}\left\{{ }^{1} \mathrm{H}\right\}$ NMR $\left(119 \mathrm{MHz}, \mathrm{CDCl}_{3}\right): \delta 18.2$.

HRMS (ESI) m/z: [M + Na ${ }^{+}$Calcd for $\mathrm{C}_{18} \mathrm{H}_{24} \mathrm{O}_{2} \mathrm{SiNa}$ : 323.1438; Found: 323.1437.

(4-methoxybenzyloxy)dimethyl(3-chloropropyl)silane (4h) 


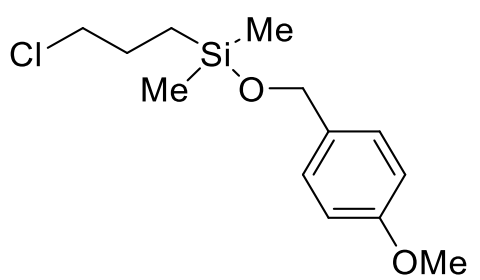

Purified by silica gel chromatography (eluent; hexane/AcOEt $=30 / 1$ ), obtained as colorless oil ( $86 \%$ yield, $116.9 \mathrm{mg})$.

${ }^{1} \mathrm{H}$ NMR (600 MHz, $\left.\mathrm{CDCl}_{3}\right): \delta 7.24(\mathrm{~d}, J=8.7 \mathrm{~Hz}, 2 \mathrm{H}, \mathrm{Ar}), 6.87(\mathrm{~d}, J=8.7 \mathrm{~Hz}, 2 \mathrm{H}, \mathrm{Ar})$, $4.63\left(\mathrm{~s}, 2 \mathrm{H}, \mathrm{OC}_{2} \mathrm{Ar}\right), 3.80\left(\mathrm{~s}, 3 \mathrm{H}, \mathrm{OC}_{3}\right), 3.49\left(\mathrm{t}, J=6.9 \mathrm{~Hz}, 2 \mathrm{H}, \mathrm{ClC}_{2}\right), 1.80(\mathrm{~m}, 2 \mathrm{H}$, $\left.\mathrm{CH}_{2} \underline{\mathrm{CH}}_{2} \mathrm{CH}_{2}\right), 0.72\left(\mathrm{~m}, 2 \mathrm{H}, \mathrm{SiCH}_{2}\right), 0.14$ (s, 6H, SiC$\left.\underline{H}_{3}\right)$.

${ }^{13} \mathrm{C}\left\{{ }^{1} \mathrm{H}\right\}$ NMR $\left(150 \mathrm{MHz}, \mathrm{CDCl}_{3}\right): \delta 158.9,132.9,128.1,113.8,64.6,55.3,47.9,26.9$, $14.1,-2.0$.

${ }^{29} \mathrm{Si}\left\{{ }^{1} \mathrm{H}\right\} \mathrm{NMR}\left(119 \mathrm{MHz}, \mathrm{CDCl}_{3}\right): \delta 18.2$.

HRMS (ESI) m/z: [M + Na] ${ }^{+}$Calcd for $\mathrm{C}_{13} \mathrm{H}_{21} \mathrm{ClO}_{2} \mathrm{SiNa}$ : 295.0892; Found: 295.0900 .

\section{(4-methoxybenzyloxy)dimethyl(3,3,3-trifluoropropyl)silane (4i)}

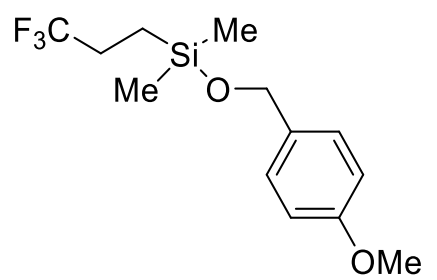

Purified by silica gel chromatography (eluent; hexane/AcOEt $=40 / 1)$, obtained as colorless oil ( $82 \%$ yield, $120.0 \mathrm{mg})$.

${ }^{1} \mathrm{H}$ NMR (600 MHz, $\left.\mathrm{CDCl}_{3}\right): \delta 7.23(\mathrm{~d}, J=8.7 \mathrm{~Hz}, 2 \mathrm{H}, \mathrm{Ar}), 6.88(\mathrm{~d}, J=8.7 \mathrm{~Hz}, 2 \mathrm{H}, \mathrm{Ar})$,

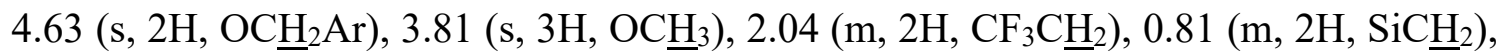
$0.16\left(\mathrm{~s}, 6 \mathrm{H}, \mathrm{SiC}_{3}\right)$.

${ }^{13} \mathrm{C}\left\{{ }^{1} \mathrm{H}\right\}$ NMR $\left(150 \mathrm{MHz}, \mathrm{CDCl}_{3}\right): \delta 159.0,132.6,128.2,127.7\left(\mathrm{q},{ }^{1} J_{\mathrm{C}-\mathrm{F}}=276.4 \mathrm{~Hz}\right)$, $113.8,64.7,55.3,28.1\left(\mathrm{q},{ }^{2} J_{\mathrm{C}-\mathrm{F}}=30.0 \mathrm{~Hz}\right), 8.3\left(\mathrm{q},{ }^{3} J_{\mathrm{C}-\mathrm{F}}=1.9 \mathrm{~Hz}\right),-2.2$.

${ }^{29} \mathrm{Si}\left\{{ }^{1} \mathrm{H}\right\}$ NMR $\left(119 \mathrm{MHz}, \mathrm{CDCl}_{3}\right): \delta 17.5$.

HRMS (ESI) m/z: [M + Na $]^{+}$Calcd for $\mathrm{C}_{13} \mathrm{H}_{19} \mathrm{~F}_{3} \mathrm{O}_{2} \mathrm{SiNa}$ : 315.0999; Found: 315.0987.

(4-methoxybenzyloxy)dimethyl(cyclohexyl)silane $(4 \mathbf{j})$ 


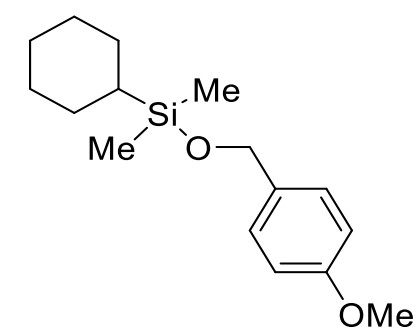

Purified by silica gel chromatography (eluent; hexane/AcOEt $=20 / 1$ ), obtained as colorless oil (91\% yield, 126.1mg).

${ }^{1} \mathrm{H} \mathrm{NMR}\left(600 \mathrm{MHz}, \mathrm{CDCl}_{3}\right): \delta 7.24(\mathrm{~d}, J=8.7 \mathrm{~Hz}, 2 \mathrm{H}, \mathrm{Ar}), 6.87(\mathrm{~d}, J=8.7 \mathrm{~Hz}, 2 \mathrm{H}, \mathrm{Ar})$, $4.63\left(\mathrm{~s}, 2 \mathrm{H}, \mathrm{OCH}_{2} \mathrm{Ar}\right), 3.80\left(\mathrm{~s}, 3 \mathrm{H}, \mathrm{OC}_{3}\right), 1.80-1.60(\mathrm{~m}, 5 \mathrm{H}, \mathrm{Cy}), 1.30-1.00(\mathrm{~m}, 5 \mathrm{H}$,

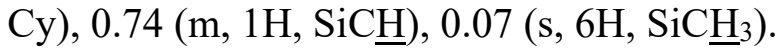

${ }^{13} \mathrm{C}\left\{{ }^{1} \mathrm{H}\right\}$ NMR $\left(150 \mathrm{MHz}, \mathrm{CDCl}_{3}\right): \delta 158.7,133.4,127.9,113.7,64.5,55.3,27.9,27.0$, $26.8,26.7,-4.0$.

${ }^{29} \mathrm{Si}\left\{{ }^{1} \mathrm{H}\right\} \mathrm{NMR}\left(119 \mathrm{MHz}, \mathrm{CDCl}_{3}\right): \delta 17.9$.

HRMS (ESI) m/z: [M + Na] ${ }^{+}$Calcd for $\mathrm{C}_{16} \mathrm{H}_{26} \mathrm{O}_{2} \mathrm{SiNa}$ : 301.1594; Found: 301.1604.

\section{(4-methoxybenzyloxy)dimethyl(phenyl)silane (4k)}

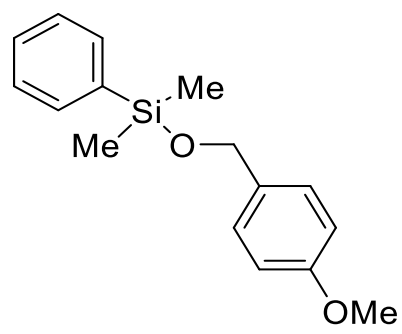

Purified by silica gel chromatography (eluent; hexane/AcOEt $=30 / 1$ ), obtained as colorless oil ( $85 \%$ yield, $115.2 \mathrm{mg})$.

${ }^{1} \mathrm{H}$ NMR (600 MHz, $\mathrm{CDCl}_{3}$ ): $\delta 7.60$ (m, 2H, Ar), 7.40 (m, 3H, Ar), 7.21 (d, J=8.7 Hz, $2 \mathrm{H}, \mathrm{Ar}), 6.86(\mathrm{~d}, J=8.7 \mathrm{~Hz}, 2 \mathrm{H}, \mathrm{Ar}), 4.63\left(\mathrm{~s}, 2 \mathrm{H}, \mathrm{OC}_{2} \mathrm{Ar}\right), 3.80$ (s, 3H, OC$\left.\underline{H}_{3}\right), 0.40$ (s, $\left.6 \mathrm{H}, \mathrm{SiC}_{3}\right)$.

${ }^{13} \mathrm{C}\left\{{ }^{1} \mathrm{H}\right\}$ NMR $\left(150 \mathrm{MHz}, \mathrm{CDCl}_{3}\right): \delta 158.9,137.7,133.6,132.9,129.7,128.2,127.9$, $113.7,64.8,55.3,-1.6$.

${ }^{29} \mathrm{Si}\left\{{ }^{1} \mathrm{H}\right\}$ NMR $\left(119 \mathrm{MHz}, \mathrm{CDCl}_{3}\right): \delta 8.5$.

HRMS (ESI) m/z: $[\mathrm{M}+\mathrm{Na}]^{+}$Calcd for $\mathrm{C}_{16} \mathrm{H}_{20} \mathrm{O}_{2} \mathrm{SiNa}$ : 295.1125; Found: 295.1126.

(benzhydryloxy)dimethyl(phenylethenyl)silane (4l) 


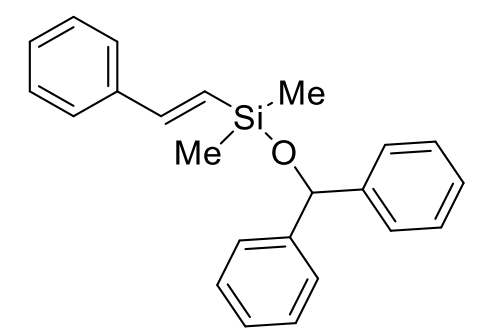

Purified by silica gel chromatography (eluent; hexane/AcOEt $=30 / 1$ ), obtained as colorless oil (63\% yield, $109.0 \mathrm{mg})$.

${ }^{1} \mathrm{H}$ NMR (600 MHz, $\left.\mathrm{CDCl}_{3}\right): \delta$ 7.42-7.18 (m, 15H, Ph), $6.90(\mathrm{dd}, J=19.2,2.0 \mathrm{~Hz}, 1 \mathrm{H}$, $\mathrm{PhC} \underline{\mathrm{H}}=\mathrm{CH}), 6.32(\mathrm{dd}, J=19.2,2.6 \mathrm{~Hz}, 1 \mathrm{H}, \mathrm{PhCH}=\mathrm{C} \underline{\mathrm{H}}), 5.83(\mathrm{~s}, 1 \mathrm{H}, \mathrm{OC} \underline{\mathrm{H}}), 0.22(\mathrm{~s}, 6 \mathrm{H}$, $\left.\mathrm{SiC}_{3}\right)$.

${ }^{13} \mathrm{C}\left\{{ }^{1} \mathrm{H}\right\}$ NMR $\left(150 \mathrm{MHz}, \mathrm{CDCl}_{3}\right): \delta 145.6,144.7,138.0,128.5,128.3,128.2,127.1$, $126.64,126.61,126.55,76.8,-1.1$.

${ }^{29} \mathrm{Si}\left\{{ }^{1} \mathrm{H}\right\}$ NMR $\left(119 \mathrm{MHz}, \mathrm{CDCl}_{3}\right): \delta 7.7$.

HRMS (ESI) m/z: $[\mathrm{M}+\mathrm{Na}]^{+}$Calcd for $\mathrm{C}_{23} \mathrm{H}_{24} \mathrm{OSiNa}$ : 367.1489; Found: 367.1496.

\section{(4-methoxybenzyloxy)methyl(diethyl)silane $(4 \mathrm{~m})$}

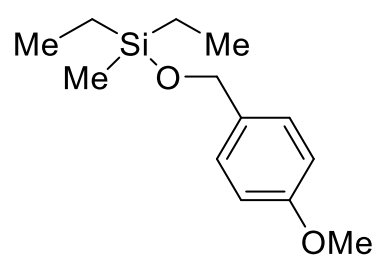

Purified by silica gel chromatography (eluent; hexane/AcOEt $=20 / 1$ ), obtained as colorless oil (95\% yield, $113.3 \mathrm{mg})$.

${ }^{1} \mathrm{H}$ NMR (600 MHz, $\left.\mathrm{CDCl}_{3}\right): \delta 7.25(\mathrm{~d}, J=8.7 \mathrm{~Hz}, 2 \mathrm{H}, \mathrm{Ar}), 6.87$ (d, $\left.J=8.7 \mathrm{~Hz}, 2 \mathrm{H}, \mathrm{Ar}\right)$, $4.64\left(\mathrm{~s}, 2 \mathrm{H}, \mathrm{OC}_{2} \mathrm{Ar}\right), 3.80\left(\mathrm{~s}, 3 \mathrm{H}, \mathrm{OC}_{3}\right), 0.96$ (t, $\left.J=7.9 \mathrm{~Hz}, 6 \mathrm{H}, \mathrm{CH}_{2} \mathrm{C}_{3}\right), 0.62$ (m, 4H, $\left.\mathrm{SiC}_{2}\right), 0.09$ (s, 3H, $\mathrm{SiC}_{3}$ ).

${ }^{13} \mathrm{C}\left\{{ }^{1} \mathrm{H}\right\}$ NMR $\left(150 \mathrm{MHz}, \mathrm{CDCl}_{3}\right): \delta 158.8,133.4,127.9,113.7,64.5,55.3,6.8,6.3,-$ 4.8 .

${ }^{29} \mathrm{Si}\left\{{ }^{1} \mathrm{H}\right\}$ NMR $\left(119 \mathrm{MHz}, \mathrm{CDCl}_{3}\right): \delta 20.5$.

HRMS (ESI) m/z: $[\mathrm{M}+\mathrm{Na}]^{+}$Calcd for $\mathrm{C}_{13} \mathrm{H}_{22} \mathrm{O}_{2} \mathrm{SiNa}$ : 261.1281; Found: 261.1286.

(4-methoxybenzyloxy)methyl(di-n-butyl)silane (4n) 


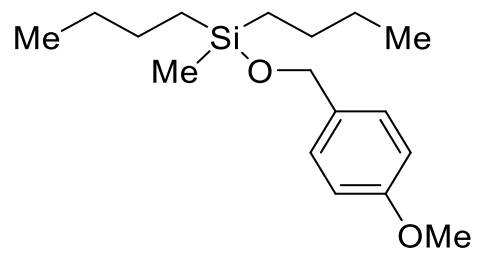

Purified by silica gel chromatography (eluent; hexane/AcOEt $=30 / 1$ ), obtained as colorless oil ( $88 \%$ yield, $129.2 \mathrm{mg})$.

${ }^{1} \mathrm{H}$ NMR (600 MHz, $\left.\mathrm{CDCl}_{3}\right): \delta 7.24(\mathrm{~d}, J=8.8 \mathrm{~Hz}, 2 \mathrm{H}, \mathrm{Ar}), 6.87(\mathrm{~d}, J=8.7 \mathrm{~Hz}, 2 \mathrm{H}, \mathrm{Ar})$, $4.63\left(\mathrm{~s}, 2 \mathrm{H}, \mathrm{OC}_{2} \mathrm{Ar}\right), 3.80\left(\mathrm{~s}, 3 \mathrm{H}, \mathrm{OCH}_{3}\right), 1.35-1.26(\mathrm{~m}, 8 \mathrm{H}$, butyl), $0.88(\mathrm{t}, J=7.1 \mathrm{~Hz}$,

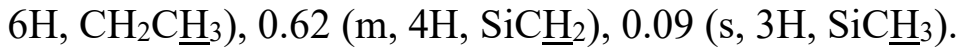

${ }^{13} \mathrm{C}\left\{{ }^{1} \mathrm{H}\right\}$ NMR $\left(150 \mathrm{MHz}, \mathrm{CDCl}_{3}\right): \delta 158.8,133.4,127.9,113.7,64.4,55.3,26.5,25.4$, $14.8,13.8,-3.8$.

${ }^{29} \mathrm{Si}\left\{{ }^{1} \mathrm{H}\right\}$ NMR $\left(119 \mathrm{MHz}, \mathrm{CDCl}_{3}\right): \delta 18.7$.

HRMS (ESI) m/z: [M + Na ${ }^{+}$Calcd for $\mathrm{C}_{17} \mathrm{H}_{30} \mathrm{O}_{2} \mathrm{SiNa}$ : 317.1907; Found: 317.1909 .

\section{(4-methoxybenzyloxy)methyl(di- $n$-pentyl)silane (4o)}

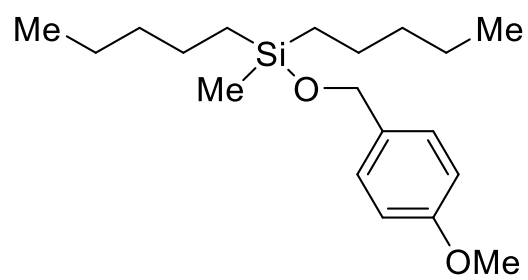

Purified by silica gel chromatography (eluent; hexane/AcOEt $=20 / 1$ ), obtained as colorless oil ( $96 \%$ yield, $155.5 \mathrm{mg})$.

${ }^{1} \mathrm{H}$ NMR (600 MHz, $\left.\mathrm{CDCl}_{3}\right): \delta 7.24(\mathrm{~d}, J=8.7 \mathrm{~Hz}, 2 \mathrm{H}, \mathrm{Ar}), 6.86(\mathrm{~d}, J=8.7 \mathrm{~Hz}, 2 \mathrm{H}, \mathrm{Ar})$, $4.63\left(\mathrm{~s}, 2 \mathrm{H}, \mathrm{OC}_{2} \mathrm{Ar}\right), 3.80\left(\mathrm{~s}, 3 \mathrm{H}, \mathrm{OC}_{3}\right), 1.40-1.20(\mathrm{~m}, 12 \mathrm{H}$, pentyl), 0.87 (t, $J=7.0$

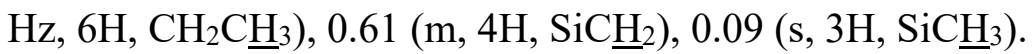

${ }^{13} \mathrm{C}\left\{{ }^{1} \mathrm{H}\right\}$ NMR $\left(150 \mathrm{MHz}, \mathrm{CDCl}_{3}\right): \delta 158.8,133.4,127.9,113.7,64.4,55.3,35.8,22.8$, $22.3,15.1,14.0,-3.8$.

${ }^{29} \mathrm{Si}\left\{{ }^{1} \mathrm{H}\right\} \mathrm{NMR}\left(119 \mathrm{MHz}, \mathrm{CDCl}_{3}\right): \delta$ 18.6.

HRMS (ESI) m/z: $[\mathrm{M}+\mathrm{Na}]^{+}$Calcd for $\mathrm{C}_{19} \mathrm{H}_{34} \mathrm{O}_{2} \mathrm{SiNa}$ : 345.2220; Found: 345.2213.

(4-methoxybenzyloxy)methyl(di-n-hexyl)silane (4p) 


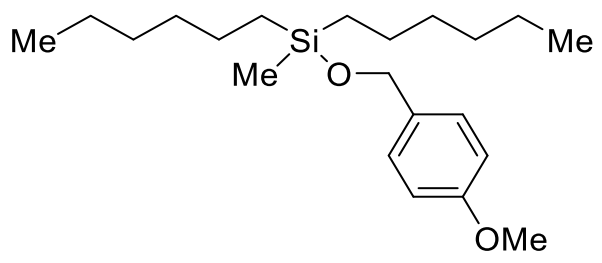

Purified by silica gel chromatography (eluent; hexane/AcOEt $=20 / 1$ ), obtained as colorless oil (94\% yield, $165.1 \mathrm{mg})$.

${ }^{1} \mathrm{H}$ NMR (600 MHz, $\left.\mathrm{CDCl}_{3}\right): \delta 7.24(\mathrm{~d}, J=8.2 \mathrm{~Hz}, 2 \mathrm{H}, \mathrm{Ar}), 6.86(\mathrm{~d}, J=8.2 \mathrm{~Hz}, 2 \mathrm{H}, \mathrm{Ar})$, $4.63\left(\mathrm{~s}, 2 \mathrm{H}, \mathrm{OC}_{2} \mathrm{Ar}\right), 3.80\left(\mathrm{~s}, 3 \mathrm{H}, \mathrm{OCH}_{3}\right), 1.40-1.15(\mathrm{~m}, 16 \mathrm{H}$, hexyl), $0.88(\mathrm{t}, J=7.2$

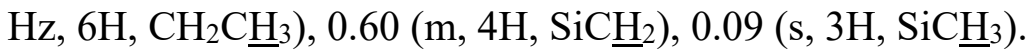

${ }^{13} \mathrm{C}\left\{{ }^{1} \mathrm{H}\right\} \mathrm{NMR}\left(150 \mathrm{MHz}, \mathrm{CDCl}_{3}\right): \delta 158.8,133.4,128.0,113.7,64.4,55.3,33.2,31.6$, $23.2,22.6,15.1,14.2,-3.8$.

${ }^{29} \mathrm{Si}\left\{{ }^{1} \mathrm{H}\right\} \mathrm{NMR}\left(119 \mathrm{MHz}, \mathrm{CDCl}_{3}\right): \delta 18.6$.

HRMS (ESI) m/z: $[\mathrm{M}+\mathrm{Na}]^{+}$Calcd for $\mathrm{C}_{21} \mathrm{H}_{38} \mathrm{O}_{2} \mathrm{SiNa}$ : 373.2533; Found: 373.2538.

\section{(4-methoxybenzyloxy)methyl(di-n-octyl)silane (4q)}

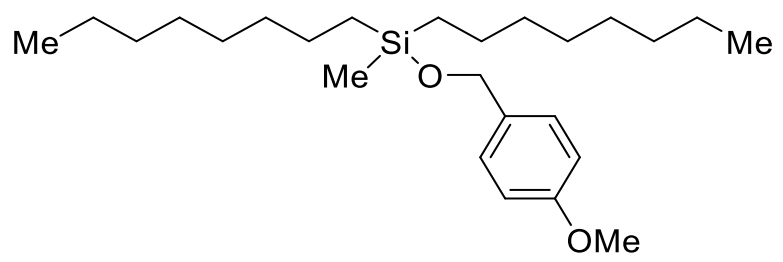

Purified by silica gel chromatography (eluent; hexane/AcOEt $=30 / 1$ ), obtained as colorless oil ( $80 \%$ yield, $161.9 \mathrm{mg})$.

${ }^{1} \mathrm{H}$ NMR (600 MHz, $\left.\mathrm{CDCl}_{3}\right): \delta 7.24(\mathrm{~d}, J=8.7 \mathrm{~Hz}, 2 \mathrm{H}, \mathrm{Ar}), 6.86(\mathrm{~d}, J=8.7 \mathrm{~Hz}, 2 \mathrm{H}, \mathrm{Ar})$, $4.63\left(\mathrm{~s}, 2 \mathrm{H}, \mathrm{OC}_{2} \mathrm{Ar}\right), 3.80$ (s, 3H, OC$\left.{ }_{3}\right), 1.40-1.20$ (m, 24H, octyl), 0.88 (t, $J=7.1$

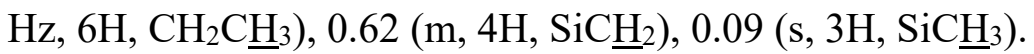

${ }^{13} \mathrm{C}\left\{{ }^{1} \mathrm{H}\right\}$ NMR $\left(150 \mathrm{MHz}, \mathrm{CDCl}_{3}\right): \delta 158.8,133.4,128.0,113.7,64.4,55.3,33.6,32.0$, $29.31,29.28,23.2,22.7,15.1,14.1,-3.8$.

${ }^{29} \mathrm{Si}\left\{{ }^{1} \mathrm{H}\right\} \mathrm{NMR}\left(119 \mathrm{MHz}, \mathrm{CDCl}_{3}\right): \delta 18.7$.

HRMS (ESI) m/z: [M + Na] ${ }^{+}$Calcd for $\mathrm{C}_{25} \mathrm{H}_{46} \mathrm{O}_{2} \mathrm{SiNa}$ 429.3159; Found: 429.3145.

(4-methoxybenzyloxy)methyl(di-2-ethylhexyl)silane (4r) 


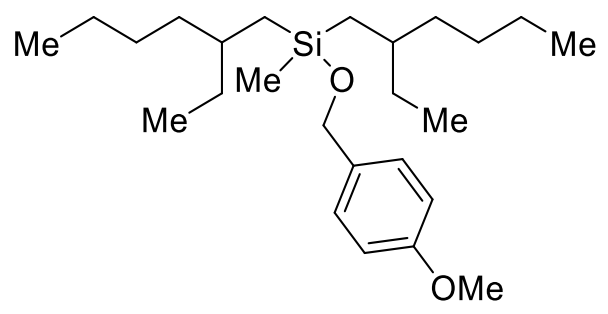

Purified by silica gel chromatography (eluent; hexane/AcOEt $=30 / 1$ ), obtained as colorless oil (77\% yield, $156.2 \mathrm{mg})$.

${ }^{1} \mathrm{H}$ NMR (600 MHz, $\left.\mathrm{CDCl}_{3}\right): \delta 7.24(\mathrm{~d}, J=8.5 \mathrm{~Hz}, 2 \mathrm{H}, \mathrm{Ar}), 6.86(\mathrm{~d}, J=8.6 \mathrm{~Hz}, 2 \mathrm{H}, \mathrm{Ar})$, $4.63\left(\mathrm{~s}, 2 \mathrm{H}, \mathrm{OCH}_{2} \mathrm{Ar}\right), 3.80\left(\mathrm{~s}, 3 \mathrm{H}, \mathrm{OCH}_{3}\right), 1.49\left(\mathrm{~m}, 2 \mathrm{H}, \mathrm{CH}_{2} \mathrm{C} \underline{\mathrm{H}}\right), 1.40-1.15(\mathrm{~m}, 16 \mathrm{H}$, 2-ethylhexyl), 0.88 (t, $J=7.1 \mathrm{~Hz}, 6 \mathrm{H}, \mathrm{CH}_{2} \mathrm{C}_{3}$ ), 0.83 (t, $\left.J=7.4 \mathrm{~Hz}, 6 \mathrm{H}, \mathrm{CH}_{2} \underline{\mathrm{C}}_{3}\right), 0.62$ (m, 4H, $\left.\mathrm{SiC}_{2}\right), 0.15$ (s, 3H, $\left.\mathrm{SiC}_{3}\right)$.

${ }^{13} \mathrm{C}\left\{{ }^{1} \mathrm{H}\right\} \operatorname{NMR}\left(150 \mathrm{MHz}, \mathrm{CDCl}_{3}\right): \delta 158.7,133.5,127.8,113.6,64.2,55.3,35.7^{*}$, $34.8^{*}, 29.0,28.8,23.1,20.7^{*}, 14.2,10.8^{*},-2.0$. (* Splitting due to the corresponding diastereomers)

${ }^{29} \mathrm{Si}\left\{{ }^{1} \mathrm{H}\right\}$ NMR $\left(119 \mathrm{MHz}, \mathrm{CDCl}_{3}\right): \delta 18.5$.

HRMS (ESI) m/z: [M + Na] ${ }^{+}$Calcd for $\mathrm{C}_{25} \mathrm{H}_{46} \mathrm{O}_{2} \mathrm{SiNa}$ 429.3159; Found: 429.3163.

\section{(4-methoxybenzyloxy)methyl(diisopropyl)silane (4s)}

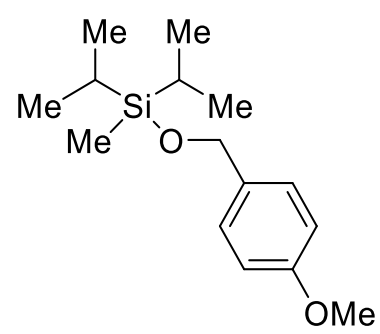

Purified by silica gel chromatography (eluent; hexane/AcOEt $=40 / 1)$, obtained as colorless oil ( $31 \%$ yield, $41.3 \mathrm{mg}$ ).

${ }^{1} \mathrm{H}$ NMR (600 MHz, $\left.\mathrm{CDCl}_{3}\right): \delta 7.25(\mathrm{~d}, J=8.7 \mathrm{~Hz}, 2 \mathrm{H}, \mathrm{Ar}), 6.87$ (d, $\left.J=8.7 \mathrm{~Hz}, 2 \mathrm{H}, \mathrm{Ar}\right)$, $4.69\left(\mathrm{~s}, 2 \mathrm{H}, \mathrm{OCH}_{2} \mathrm{Ar}\right), 3.80\left(\mathrm{~s}, 3 \mathrm{H}, \mathrm{OC}_{3}\right), 1.06-0.96(\mathrm{~m}, 14 \mathrm{H}, i-\mathrm{Pr}), 0.05(\mathrm{~s}, 3 \mathrm{H}$, $\left.\mathrm{SiC}_{3}\right)$.

${ }^{13} \mathrm{C}\left\{{ }^{1} \mathrm{H}\right\} \mathrm{NMR}\left(150 \mathrm{MHz}, \mathrm{CDCl}_{3}\right): \delta 158.7,133.6,127.6,113.6,64.8,55.3,17.5,17.4$, $13.1,-8.5$.

${ }^{29} \mathrm{Si}\left\{{ }^{1} \mathrm{H}\right\} \mathrm{NMR}\left(119 \mathrm{MHz}, \mathrm{CDCl}_{3}\right): \delta 19.3$.

HRMS (ESI) m/z: [M + Na] ${ }^{+}$Calcd for $\mathrm{C}_{15} \mathrm{H}_{26} \mathrm{O}_{2} \mathrm{SiNa}$ : 289.1594; Found: 289.1587. 
(benzhydryloxy)dimethyl(ethyl)silane (4u)

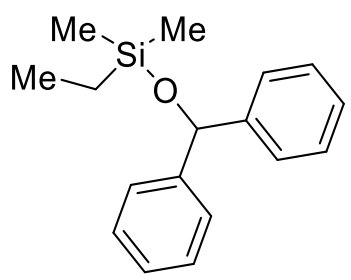

Purified by silica gel chromatography (eluent; hexane/AcOEt $=40 / 1)$, obtained as colorless oil (92\% yield, $124.0 \mathrm{mg})$.

${ }^{1} \mathrm{H}$ NMR (600 MHz, $\left.\mathrm{CDCl}_{3}\right): \delta 7.34$ (m, 4H, Ar), 7.29 (m, 4H, Ar), 7.21 (m, 2H, Ar), $5.75\left(\mathrm{~s}, 1 \mathrm{H}, \mathrm{C}_{\mathrm{HPh}}\right), 0.89$ (t, $J=8.0 \mathrm{~Hz}, 3 \mathrm{H}, \mathrm{CH}_{2} \mathrm{C}_{3}$ ), 0.54 (q, $\left.J=8.0 \mathrm{~Hz}, 2 \mathrm{H}, \mathrm{SiC}_{2}\right)$, $0.04\left(\mathrm{~s}, 6 \mathrm{H}, \mathrm{SiC}_{3}\right)$.

${ }^{13} \mathrm{C}\left\{{ }^{1} \mathrm{H}\right\}$ NMR (150 MHz, $\left.\mathrm{CDCl}_{3}\right): \delta 145.0,128.2,127.0,126.5,76.4,8.5,6.7,-2.1$. ${ }^{29} \mathrm{Si}\left\{{ }^{1} \mathrm{H}\right\} \mathrm{NMR}\left(119 \mathrm{MHz}, \mathrm{CDCl}_{3}\right): \delta 19.5$.

HRMS (ESI) m/z: [M + Na $]^{+}$Calcd for $\mathrm{C}_{17} \mathrm{H}_{22} \mathrm{OSiNa}$ : 293.1332; Found: 293.1342.

(4-methoxybenzyloxy)dimethyl(octyl)silane (4v)

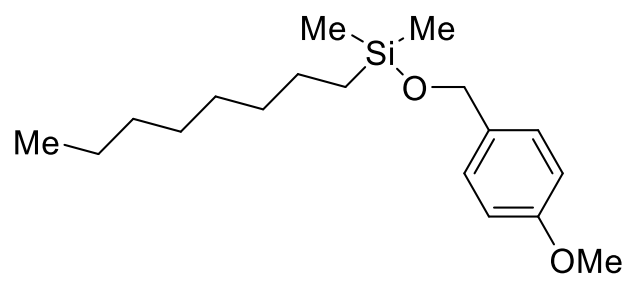

Purified by silica gel chromatography (eluent; hexane/AcOEt $=30 / 1$ ), obtained as colorless oil ( $91 \%$ yield, $140.0 \mathrm{mg}$ ).

${ }^{1} \mathrm{H}$ NMR (600 MHz, $\left.\mathrm{CDCl}_{3}\right): \delta 7.24(\mathrm{~d}, J=8.6 \mathrm{~Hz}, 2 \mathrm{H}, \mathrm{Ar}), 6.86(\mathrm{~d}, J=8.6 \mathrm{~Hz}, 2 \mathrm{H}, \mathrm{Ar})$, $4.62\left(\mathrm{~s}, 2 \mathrm{H}, \mathrm{OC}_{2} \mathrm{Ar}\right), 3.80$ (s, 3H, OC$\left.{ }_{3}\right), 1.40-1.20$ (m, 12H, octyl), 0.88 (t, $J=7.1$

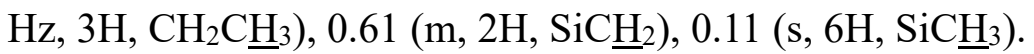

${ }^{13} \mathrm{C}\left\{{ }^{1} \mathrm{H}\right\}$ NMR $\left(150 \mathrm{MHz}, \mathrm{CDCl}_{3}\right): \delta 158.8,133.2,128.1,113.7,64.4,55.3,33.5,32.0$. 29.34, 29.27. 23.2, 22.7, 16.4, 14.1. - 2.0.

${ }^{29} \mathrm{Si}\left\{{ }^{1} \mathrm{H}\right\} \mathrm{NMR}\left(119 \mathrm{MHz}, \mathrm{CDCl}_{3}\right): \delta 18.9$.

HRMS (ESI) m/z: $[\mathrm{M}+\mathrm{Na}]^{+}$Calcd for $\mathrm{C}_{18} \mathrm{H}_{32} \mathrm{O}_{2} \mathrm{SiNa}$ : 331.2064; Found: 331.2070.

(4-methoxybenzyloxy)dimethyl(n-hexadecyl)silane (4w) 


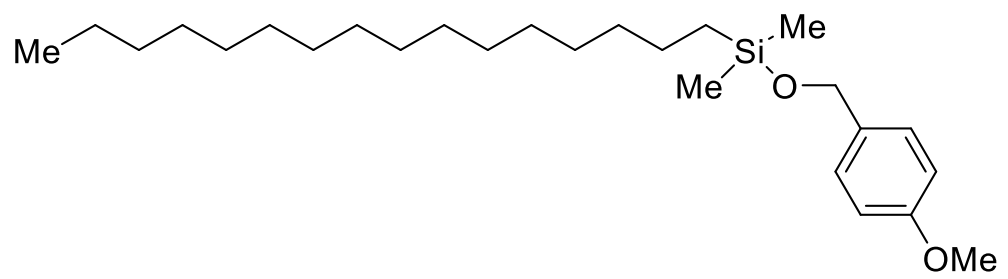

Purified by silica gel chromatography (eluent; hexane/AcOEt $=40 / 1$ ), obtained as colorless oil (93\% yield, $195.4 \mathrm{mg})$.

${ }^{1} \mathrm{H}$ NMR (600 MHz, $\left.\mathrm{CDCl}_{3}\right): \delta 7.24(\mathrm{~d}, J=8.6 \mathrm{~Hz}, 2 \mathrm{H}, \mathrm{Ar}), 6.86(\mathrm{~d}, J=8.6 \mathrm{~Hz}, 2 \mathrm{H}, \mathrm{Ar})$, $4.62\left(\mathrm{~s}, 2 \mathrm{H}, \mathrm{OC}_{2} \mathrm{Ar}\right), 3.80\left(\mathrm{~s}, 3 \mathrm{H}, \mathrm{OC}_{3}\right), 1.40-1.20(\mathrm{~m}, 28 \mathrm{H}$, hexadecyl), $0.88(\mathrm{t}, J=$ $\left.7.0 \mathrm{~Hz}, 3 \mathrm{H}, \mathrm{CH}_{2} \mathrm{CH}_{3}\right), 0.61$ (m, $\left.2 \mathrm{H}, \mathrm{SiC}_{2}\right), 0.11$ (s, $\left.6 \mathrm{H}, \mathrm{SiC}_{3}\right)$.

${ }^{13} \mathrm{C}\left\{{ }^{1} \mathrm{H}\right\}$ NMR $\left(150 \mathrm{MHz}, \mathrm{CDCl}_{3}\right): \delta 158.8,133.2,128.1,113.7,64.4,55.3,33.5,32.0$, 29.73 (6C were overlapped.), 29.69, 29.6, 29.4 (2C were overlapped.), 23.2, 22.7, 16.4, $14.2,-2.0$.

${ }^{29} \mathrm{Si}\left\{{ }^{1} \mathrm{H}\right\} \mathrm{NMR}\left(119 \mathrm{MHz}, \mathrm{CDCl}_{3}\right): \delta 18.9$.

HRMS (ESI) m/z: $[\mathrm{M}+\mathrm{Na}]^{+}$Calcd for $\mathrm{C}_{26} \mathrm{H}_{48} \mathrm{O}_{2} \mathrm{SiNa}$ : 443.3316; Found: 443.3324.

\section{(4-methoxybenzyloxy)dimethyl(isobutyl)silane (4x)}

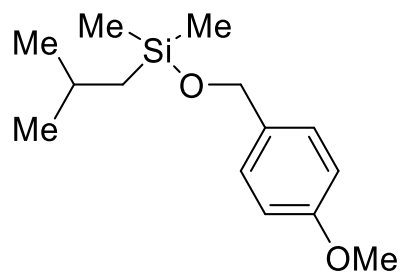

Purified by silica gel chromatography (eluent; hexane/AcOEt $=40 / 1)$, obtained as colorless oil (94\% yield, $118.6 \mathrm{mg})$.

${ }^{1} \mathrm{H}$ NMR (600 MHz, $\left.\mathrm{CDCl}_{3}\right): \delta 7.24(\mathrm{~d}, J=8.7 \mathrm{~Hz}, 2 \mathrm{H}, \mathrm{Ar}), 6.86(\mathrm{~d}, J=8.7 \mathrm{~Hz}, 2 \mathrm{H}, \mathrm{Ar})$, $4.62\left(\mathrm{~s}, 2 \mathrm{H}, \mathrm{OC}_{2} \mathrm{Ar}\right), 3.80\left(\mathrm{~s}, 3 \mathrm{H}, \mathrm{OC}_{3}\right), 1.83\left(\mathrm{~m}, 1 \mathrm{H}, \mathrm{C}^{\mathrm{HCH}} \mathrm{H}_{3}\right), 0.95(\mathrm{~d}, J=6.6 \mathrm{~Hz}$, $\left.6 \mathrm{H}, \mathrm{CHCH}_{3}\right), 0.64\left(\mathrm{~d}, J=7.0 \mathrm{~Hz}, 2 \mathrm{H}, \mathrm{SiC}_{2}\right), 0.14$ (s, 6H, $\left.\mathrm{SiC}_{3}\right)$.

${ }^{13} \mathrm{C}\left\{{ }^{1} \mathrm{H}\right\} \mathrm{NMR}\left(150 \mathrm{MHz}, \mathrm{CDCl}_{3}\right): \delta 158.8,133.3,128.0,113.7,64.3,55.3,27.0,26.2$, $24.3,-1.1$.

${ }^{29} \mathrm{Si}\left\{{ }^{1} \mathrm{H}\right\}$ NMR $\left(119 \mathrm{MHz}, \mathrm{CDCl}_{3}\right): \delta 18.2$.

HRMS (ESI) m/z: [M + Na] ${ }^{+}$Calcd for $\mathrm{C}_{14} \mathrm{H}_{24} \mathrm{O}_{2} \mathrm{SiNa}$ : 275.1438; Found: 275.1449.

(4-methoxybenzyloxy)dimethyl( $1 H, 1 H, 2 H, 2 H$-tridecafluoro-n-octyl)silane (4y) 


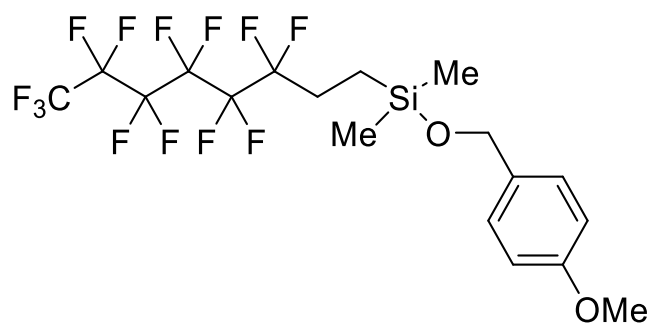

Purified by silica gel chromatography (eluent; hexane/AcOEt $=40 / 1)$, obtained as colorless oil (77\% yield, $208.8 \mathrm{mg})$.

${ }^{1} \mathrm{H}$ NMR (600 MHz, $\left.\mathrm{CDCl}_{3}\right): \delta 7.23(\mathrm{~d}, J=8.6 \mathrm{~Hz}, 2 \mathrm{H}, \mathrm{Ar}), 6.87(\mathrm{~d}, J=8.6 \mathrm{~Hz}, 2 \mathrm{H}, \mathrm{Ar})$, 4.64 (s, 2H, OC $22 \mathrm{Ar}), 3.80\left(\mathrm{~s}, 3 \mathrm{H}, \mathrm{OC}_{3}\right), 2.02\left(\mathrm{~m}, 2 \mathrm{H}, \mathrm{C}_{2} \mathrm{CF}_{2}\right), 0.81\left(\mathrm{~m}, 2 \mathrm{H}, \mathrm{SiC}_{2}\right)$, $0.17\left(\mathrm{~s}, 6 \mathrm{H}, \mathrm{SiC}_{3}\right)$.

${ }^{13} \mathrm{C}\left\{{ }^{1} \mathrm{H}\right\}$ NMR $\left(150 \mathrm{MHz}, \mathrm{CDCl}_{3}\right): \delta 159.1,132.5,128.2,113.8,64.7,55.3,25.1(\mathrm{t}$, $\left.{ }^{2} J_{\mathrm{C}-\mathrm{F}}=23.9 \mathrm{~Hz}\right), 6.0,-2.2$. (5C were not assignable due to the complicated ${ }^{13} \mathrm{C}-{ }^{19} \mathrm{~F}$ coupling.)

${ }^{29} \mathrm{Si}\left\{{ }^{1} \mathrm{H}\right\} \mathrm{NMR}\left(119 \mathrm{MHz}, \mathrm{CDCl}_{3}\right): \delta 17.9$.

HRMS (ESI) m/z: $[\mathrm{M}+\mathrm{Na}]^{+}$Calcd for $\mathrm{C}_{18} \mathrm{H}_{19} \mathrm{~F}_{13} \mathrm{O}_{2} \mathrm{SiNa}$ : 565.0839; Found: 565.0831.

\section{(4-methoxybenzyloxy)dimethyl(cyclopentyl)silane (4z)}

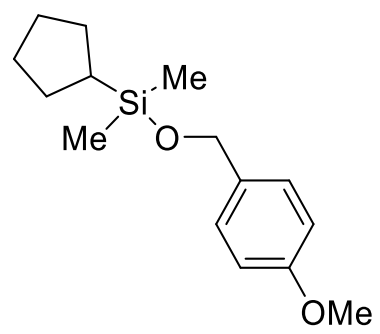

Purified by silica gel chromatography (eluent; hexane/AcOEt $=30 / 1)$, obtained as colorless oil ( $88 \%$ yield, $116.8 \mathrm{mg})$.

${ }^{1} \mathrm{H}$ NMR (600 MHz, $\left.\mathrm{CDCl}_{3}\right): \delta 7.27$ (d, $\left.J=8.8 \mathrm{~Hz}, 2 \mathrm{H}, \mathrm{Ar}\right), 6.89$ (d, $\left.J=8.8 \mathrm{~Hz}, 2 \mathrm{H}, \mathrm{Ar}\right)$, 4.68 (s, 2H, OC$\left.\underline{H}_{2} \mathrm{Ar}\right), 3.82$ (s, 3H, $\left.\mathrm{OC}_{3}\right), 1.78$ (m, 2H, Cy), 1.67-1.47 (m, 4H, Cy),

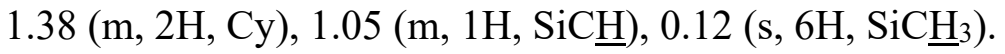

${ }^{13} \mathrm{C}\left\{{ }^{1} \mathrm{H}\right\} \mathrm{NMR}\left(150 \mathrm{MHz}, \mathrm{CDCl}_{3}\right): \delta 158.8,133.4,127.9,113.7,64.5,55.3,27.5,27.1$, $26.1,-3.5$.

${ }^{29} \mathrm{Si}\left\{{ }^{1} \mathrm{H}\right\}$ NMR $\left(119 \mathrm{MHz}, \mathrm{CDCl}_{3}\right): \delta 18.8$.

HRMS (ESI) m/z: [M + Na] ${ }^{+}$Calcd for $\mathrm{C}_{15} \mathrm{H}_{24} \mathrm{O}_{2} \mathrm{SiNa}$ : 287.1438; Found: 287.1445.

\section{(4-methoxybenzyloxy)dimethyl(n-butyl)silane (4aa)}




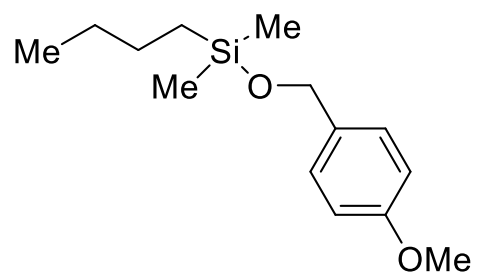

Purified by silica gel chromatography (eluent; hexane/AcOEt $=30 / 1$ ), obtained as colorless oil (90\% yield, $113.3 \mathrm{mg})$.

${ }^{1} \mathrm{H}$ NMR (600 MHz, $\left.\mathrm{CDCl}_{3}\right): \delta 7.24(\mathrm{~d}, J=7.8 \mathrm{~Hz}, 2 \mathrm{H}, \mathrm{Ar}), 6.87$ (d, $\left.J=7.8 \mathrm{~Hz}, 2 \mathrm{H}, \mathrm{Ar}\right)$, $4.62\left(\mathrm{~s}, 2 \mathrm{H}, \mathrm{OC}_{2} \mathrm{Ar}\right), 3.80\left(\mathrm{~s}, 3 \mathrm{H}, \mathrm{OC}_{3}\right), 1.31\left(\mathrm{~m}, 2 \mathrm{H}, \mathrm{CH}_{2} \mathrm{C}_{2} \mathrm{C}_{2} \mathrm{CH}_{3}\right), 0.88$ (t, $J=$ $\left.6.6 \mathrm{~Hz}, 3 \mathrm{H}, \mathrm{CH}_{2} \mathrm{C}_{3}\right), 0.62$ (m, 2H, SiC $\left.\underline{\mathrm{H}}_{2}\right), 0.11$ (s, 6H, $\left.\mathrm{SiC}_{3}\right)$.

${ }^{13} \mathrm{C}\left\{{ }^{1} \mathrm{H}\right\}$ NMR $\left(150 \mathrm{MHz}, \mathrm{CDCl}_{3}\right): \delta 158.8,133.3,128.1,113.7,64.4,55.3,26.4,25.4$, $16.1,13.8,-1.9$.

${ }^{29} \mathrm{Si}\left\{{ }^{1} \mathrm{H}\right\}$ NMR $\left(119 \mathrm{MHz}, \mathrm{CDCl}_{3}\right): \delta 18.9$.

HRMS (ESI) m/z: [M + Na] ${ }^{+}$Calcd for $\mathrm{C}_{14} \mathrm{H}_{24} \mathrm{O}_{2} \mathrm{SiNa}$ : 275.1438; Found: 275.1449. 


\section{References}

[S1] Ohmura, T.; Torigoe, T.; Suginome, M. Catalytic Functionalization of Methyl Group on Silicon: Iridium-Catalyzed $\mathrm{C}\left(\mathrm{sp}^{3}\right)-\mathrm{H}$ Borylation of Methylchlorosilanes. $J$. Am. Chem. Soc. 2012, 134, 17416-17419.

[S2] Matsumoto, K.; Huang, J.; Naganawa, Y.; Guo, H.; Beppu, T.; Sato, K.; Shimada, S.; Nakajima, Y. Direct Silyl-Heck Reaction of Chlorosilanes. Org. Lett. 2018, 20, 2481-2484. 
${ }^{1} \mathrm{H}\left(600 \mathrm{MHz}, \mathrm{CDCl}_{3}\right)$ and ${ }^{13} \mathrm{C}$ NMR Spectra (150 $\left.\mathrm{MHz}, \mathrm{CDCl}_{3}\right)$ of 2p

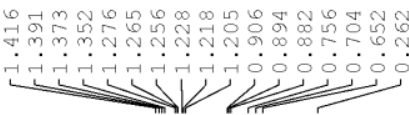

$\mathrm{Me}_{\mathrm{Me}^{\prime}{ }^{\mathrm{Cl}} \mathrm{Cl}} \overbrace{\mathrm{Me}}$

$2 p$
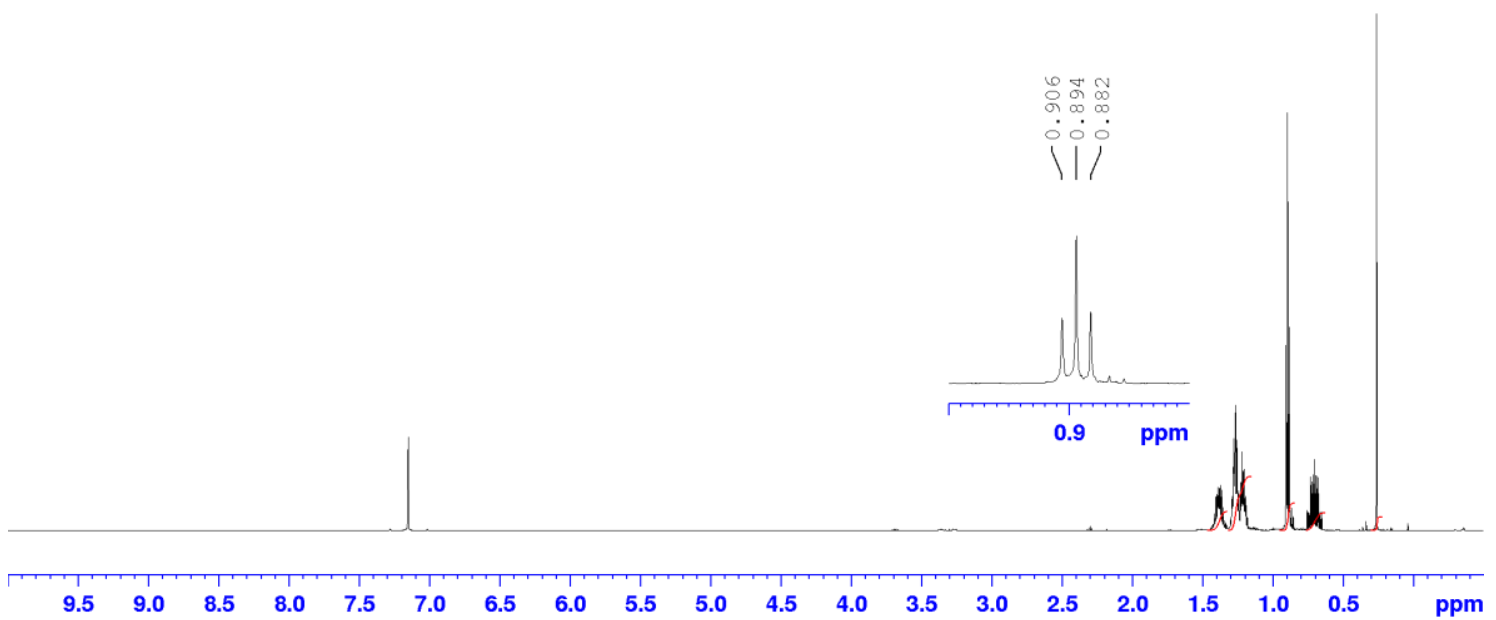

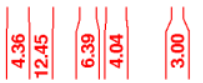
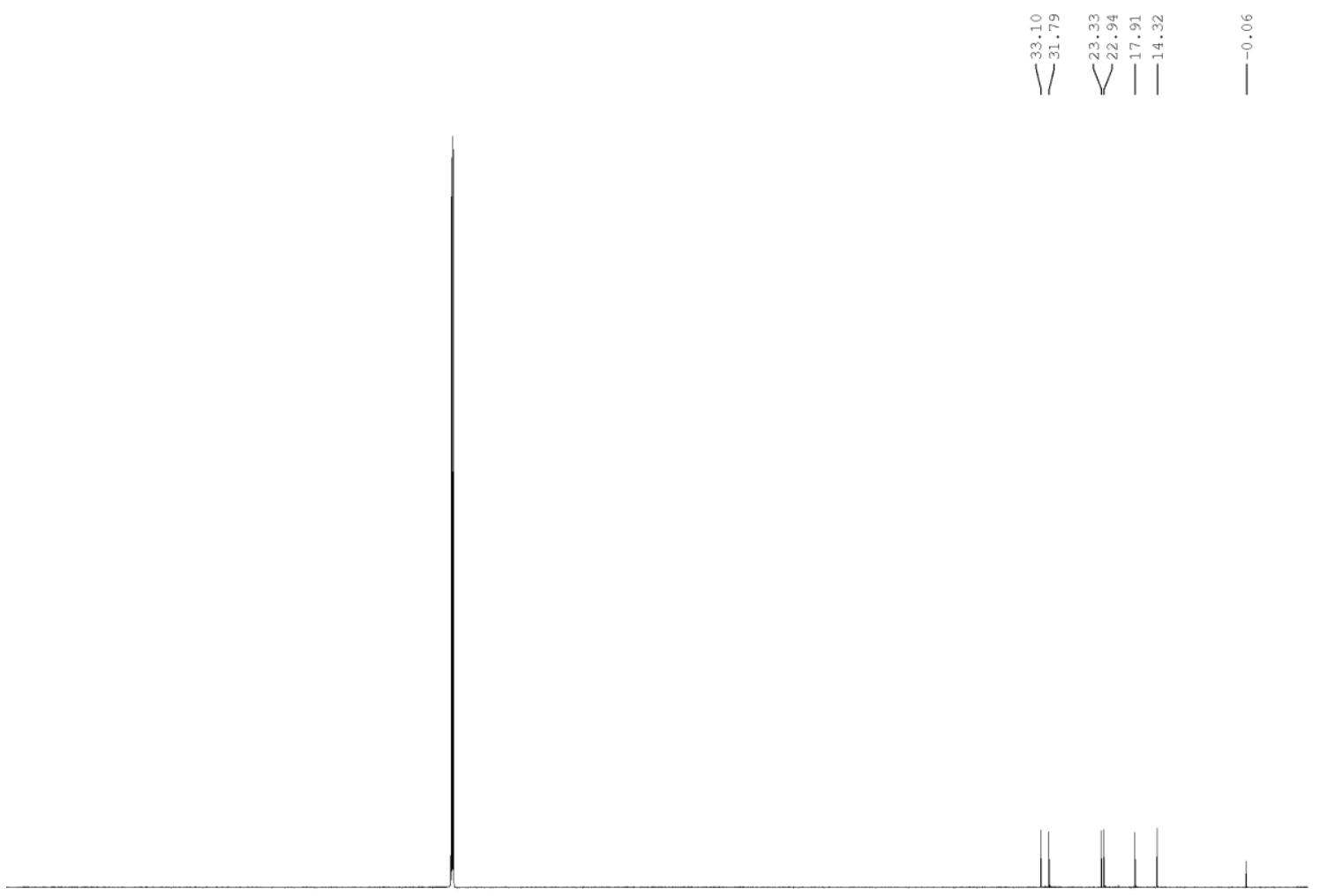

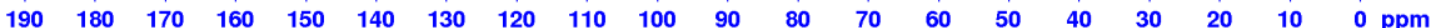


${ }^{1} \mathrm{H}\left(600 \mathrm{MHz}, \mathrm{CDCl}_{3}\right)$ and ${ }^{13} \mathrm{C}$ NMR Spectra (150 MHz, $\left.\mathrm{CDCl}_{3}\right)$ of $4 \mathrm{~b}$
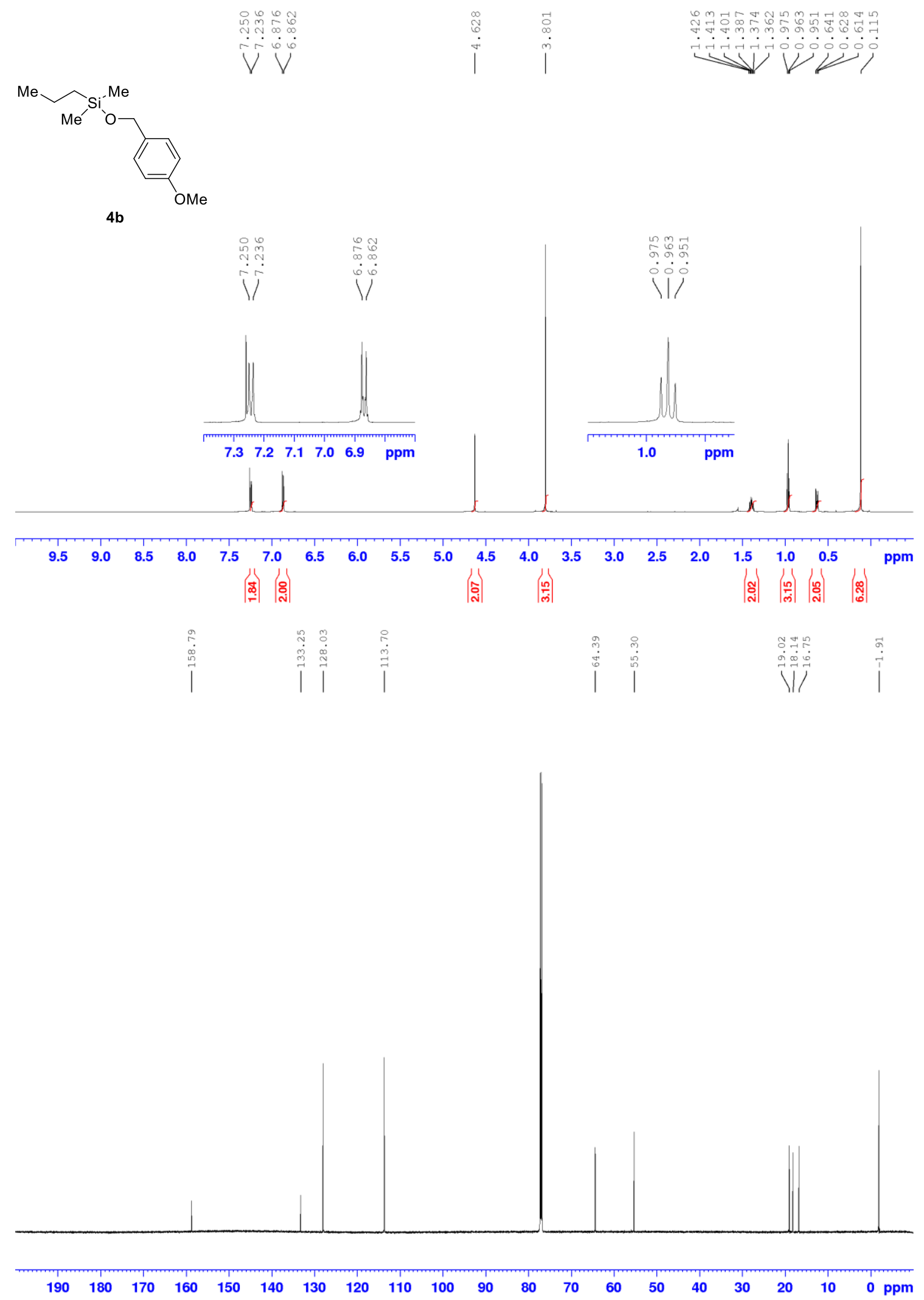
${ }^{1} \mathrm{H}\left(600 \mathrm{MHz}, \mathrm{CDCl}_{3}\right)$ and ${ }^{13} \mathrm{C}$ NMR Spectra (150 MHz, $\left.\mathrm{CDCl}_{3}\right)$ of $4 \mathrm{c}$
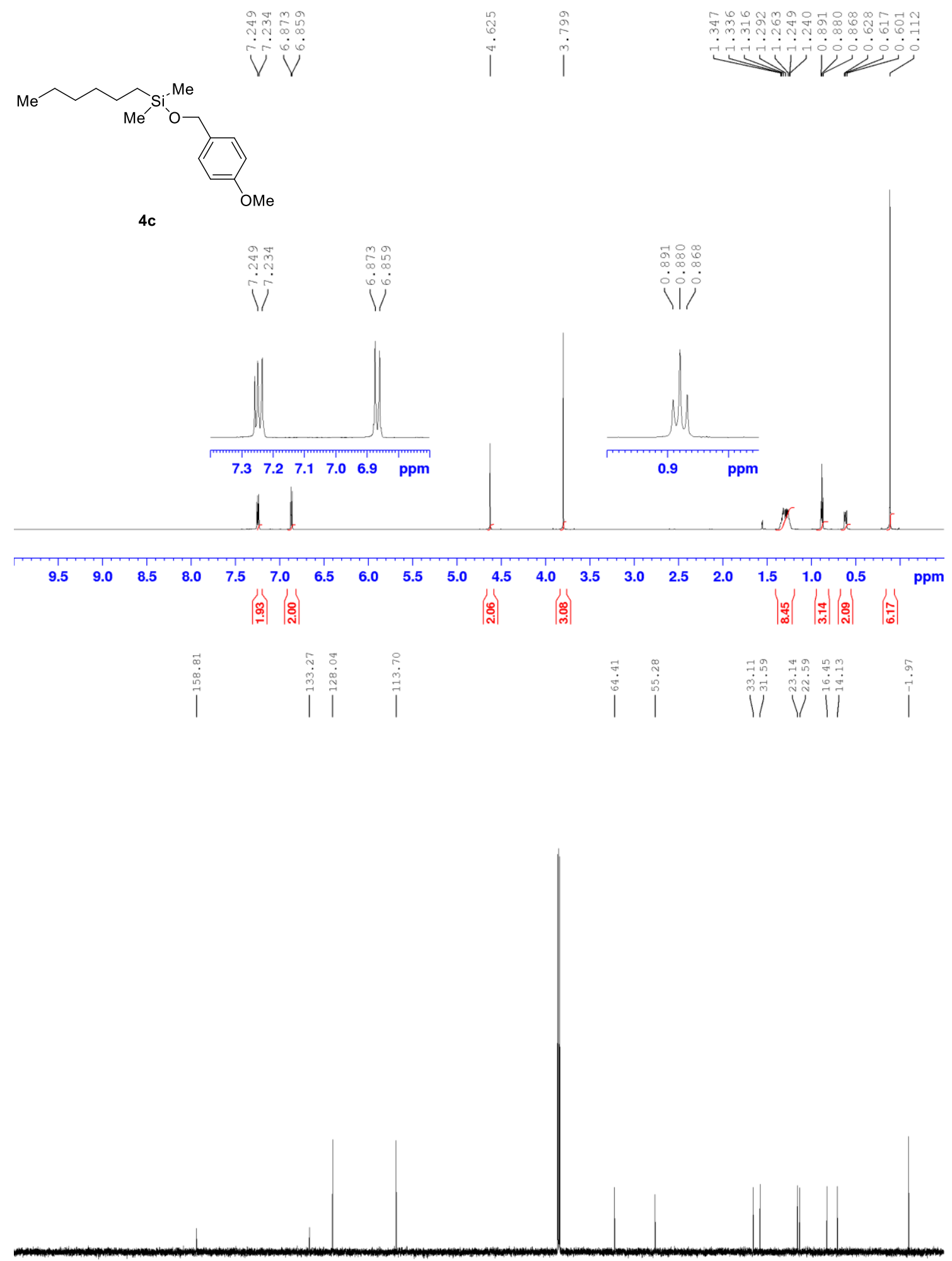

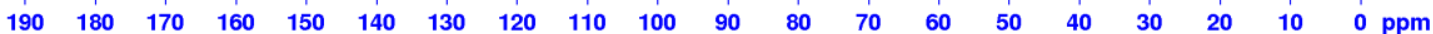


${ }^{1} \mathrm{H}\left(600 \mathrm{MHz}, \mathrm{CDCl}_{3}\right)$ and ${ }^{13} \mathrm{C}$ NMR Spectra (150 MHz, CDCl 3$)$ of 4d
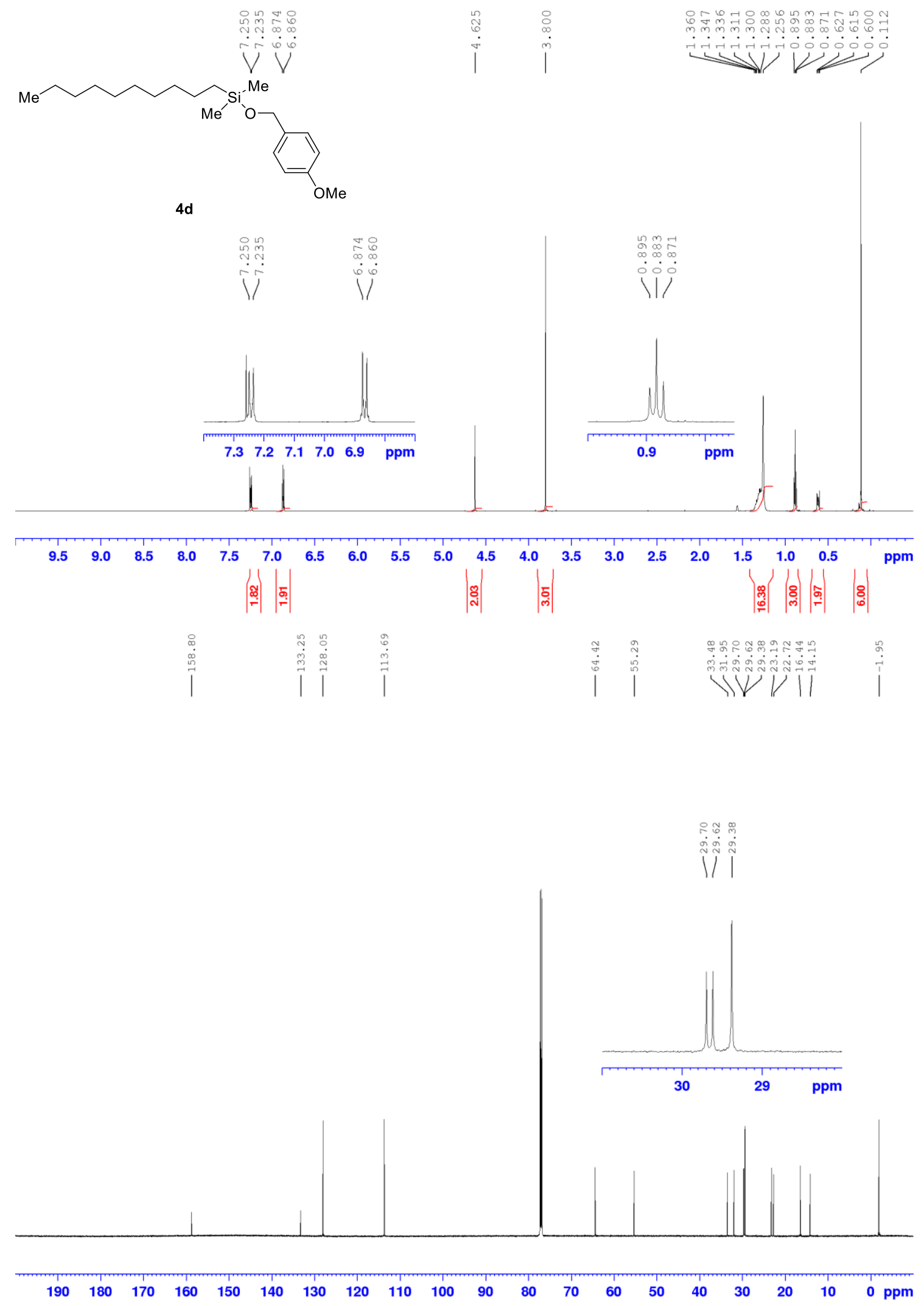
${ }^{1} \mathrm{H}\left(600 \mathrm{MHz}, \mathrm{CDCl}_{3}\right)$ and ${ }^{13} \mathrm{C}$ NMR Spectra (150 $\left.\mathrm{MHz}, \mathrm{CDCl}_{3}\right)$ of $4 \mathrm{e}$
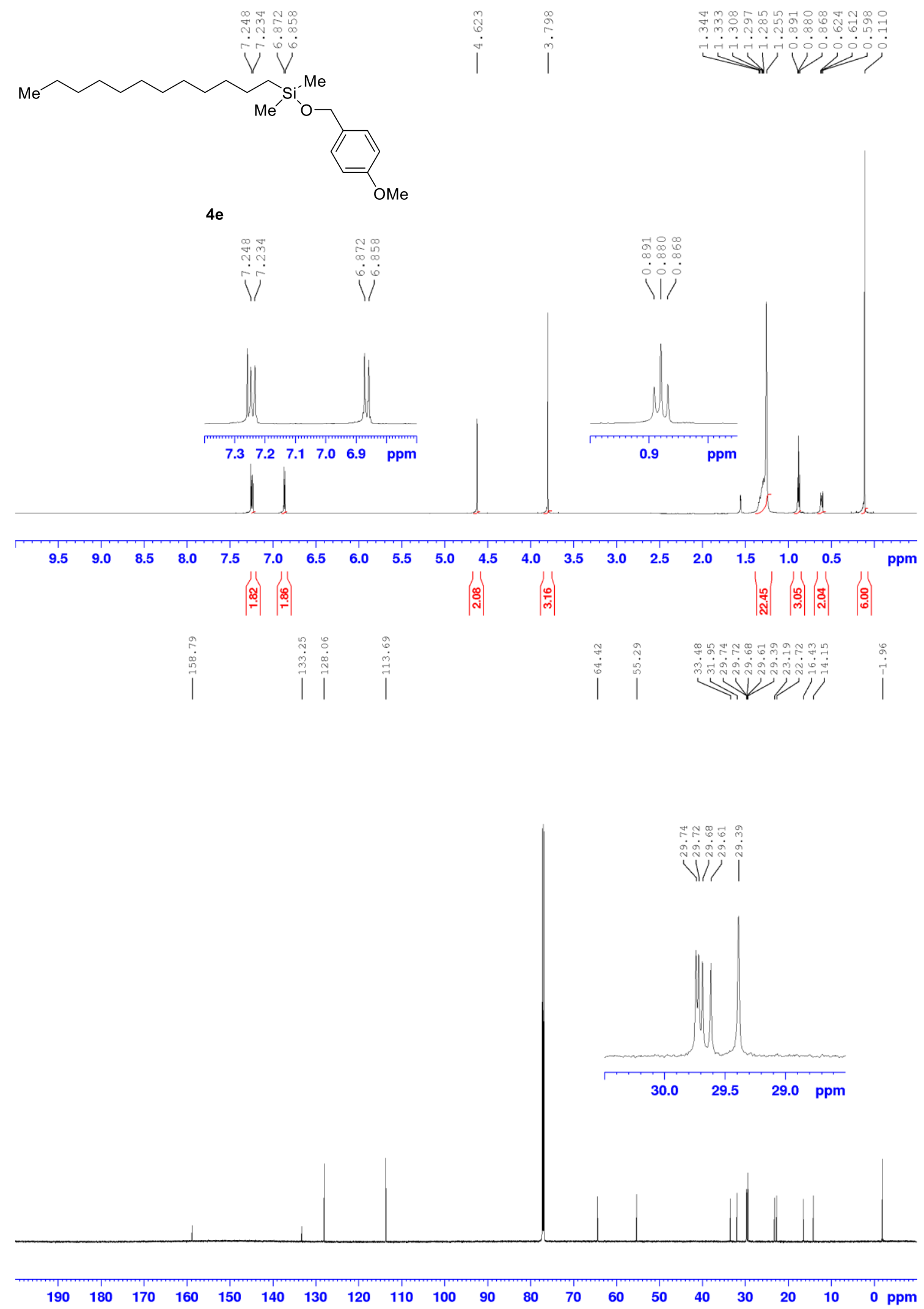
${ }^{1} \mathrm{H}\left(600 \mathrm{MHz}, \mathrm{CDCl}_{3}\right)$ and ${ }^{13} \mathrm{C}$ NMR Spectra (150 MHz, $\left.\mathrm{CDCl}_{3}\right)$ of $4 \mathrm{f}$
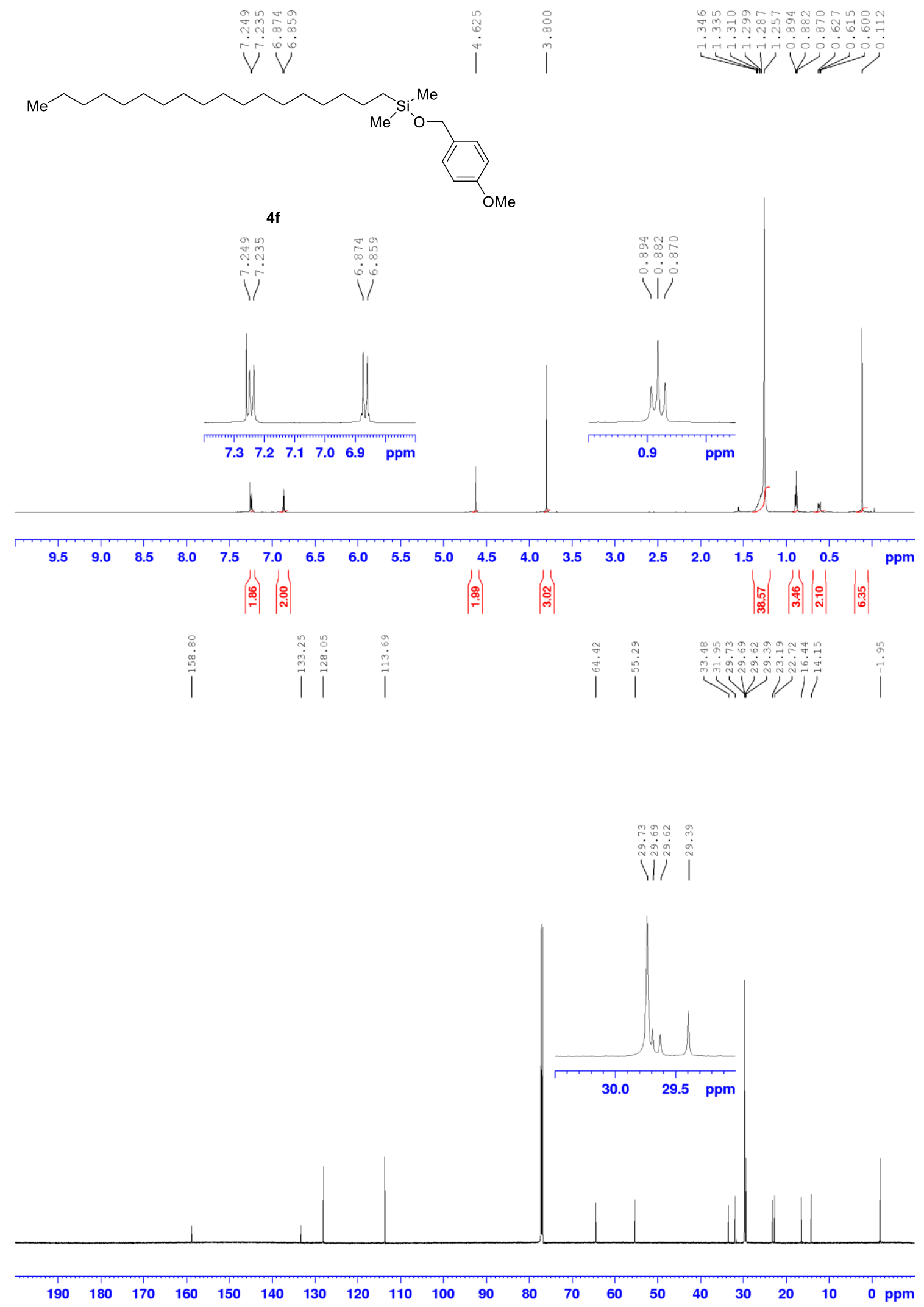
${ }^{1} \mathrm{H}\left(600 \mathrm{MHz}, \mathrm{CDCl}_{3}\right)$ and ${ }^{13} \mathrm{C}$ NMR Spectra (150 MHz, CDCl 3$)$ of $4 \mathrm{~g}$
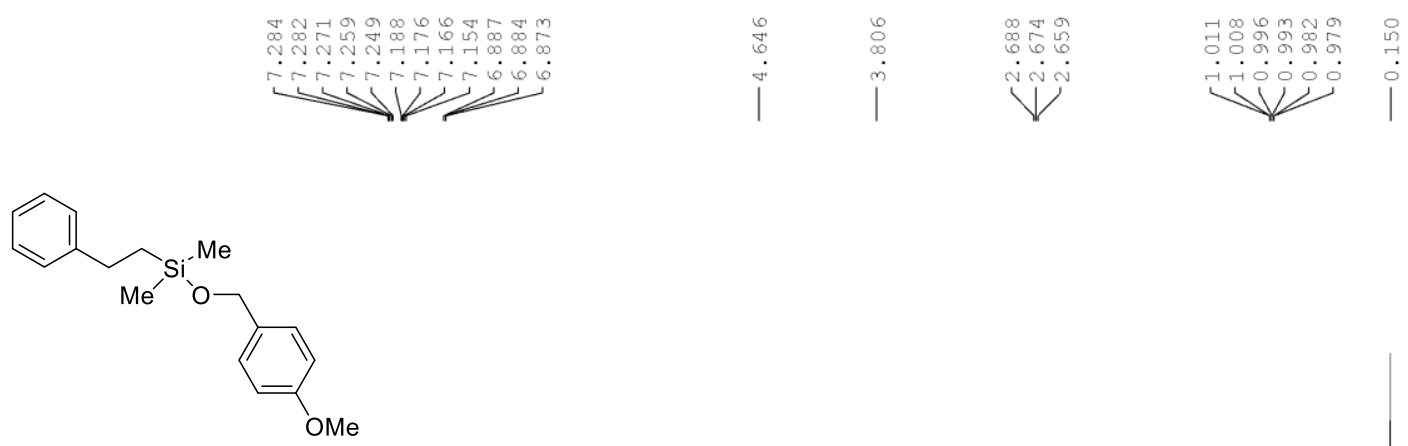

$4 \mathrm{~g}$
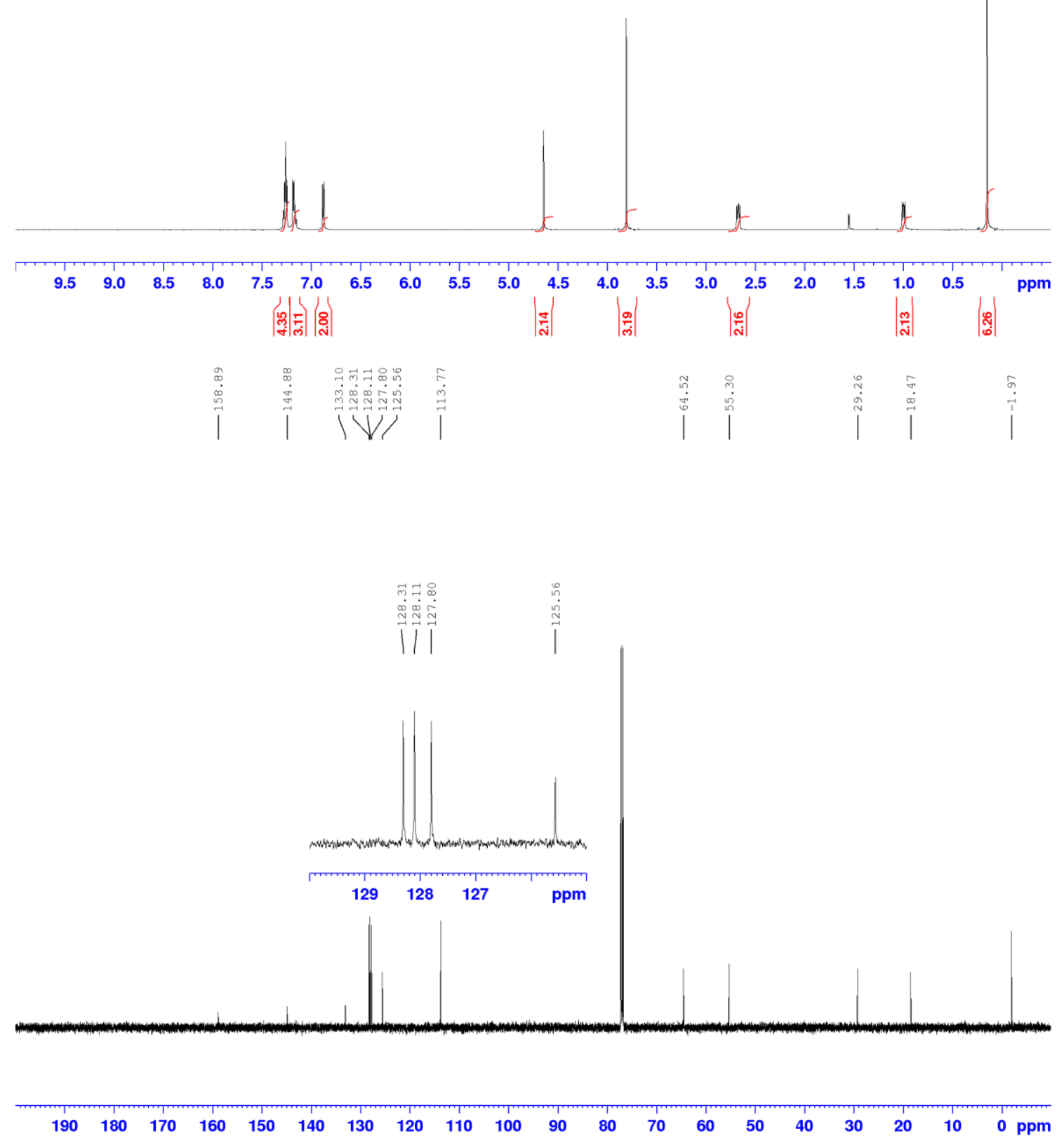

S32 
${ }^{1} \mathrm{H}\left(600 \mathrm{MHz}, \mathrm{CDCl}_{3}\right)$ and ${ }^{13} \mathrm{C}$ NMR Spectra (150 MHz, $\left.\mathrm{CDCl}_{3}\right)$ of $4 \mathrm{~h}$
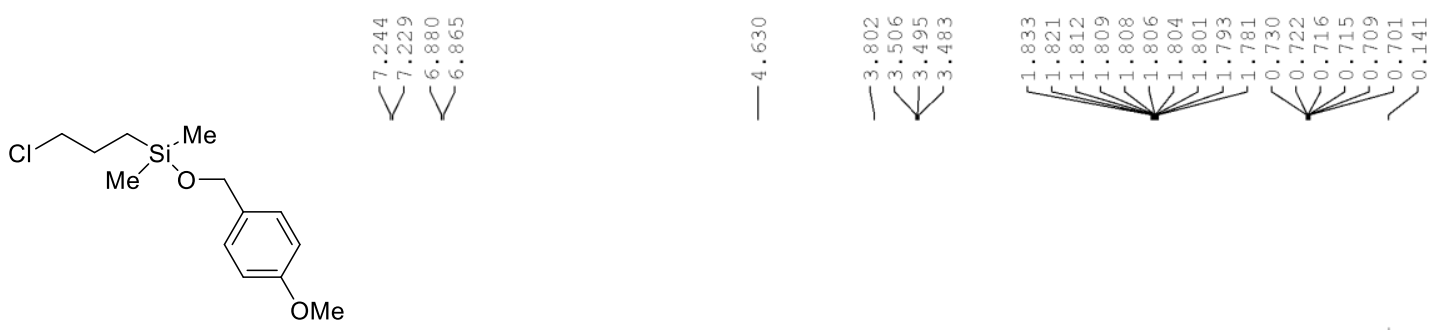

$4 h$
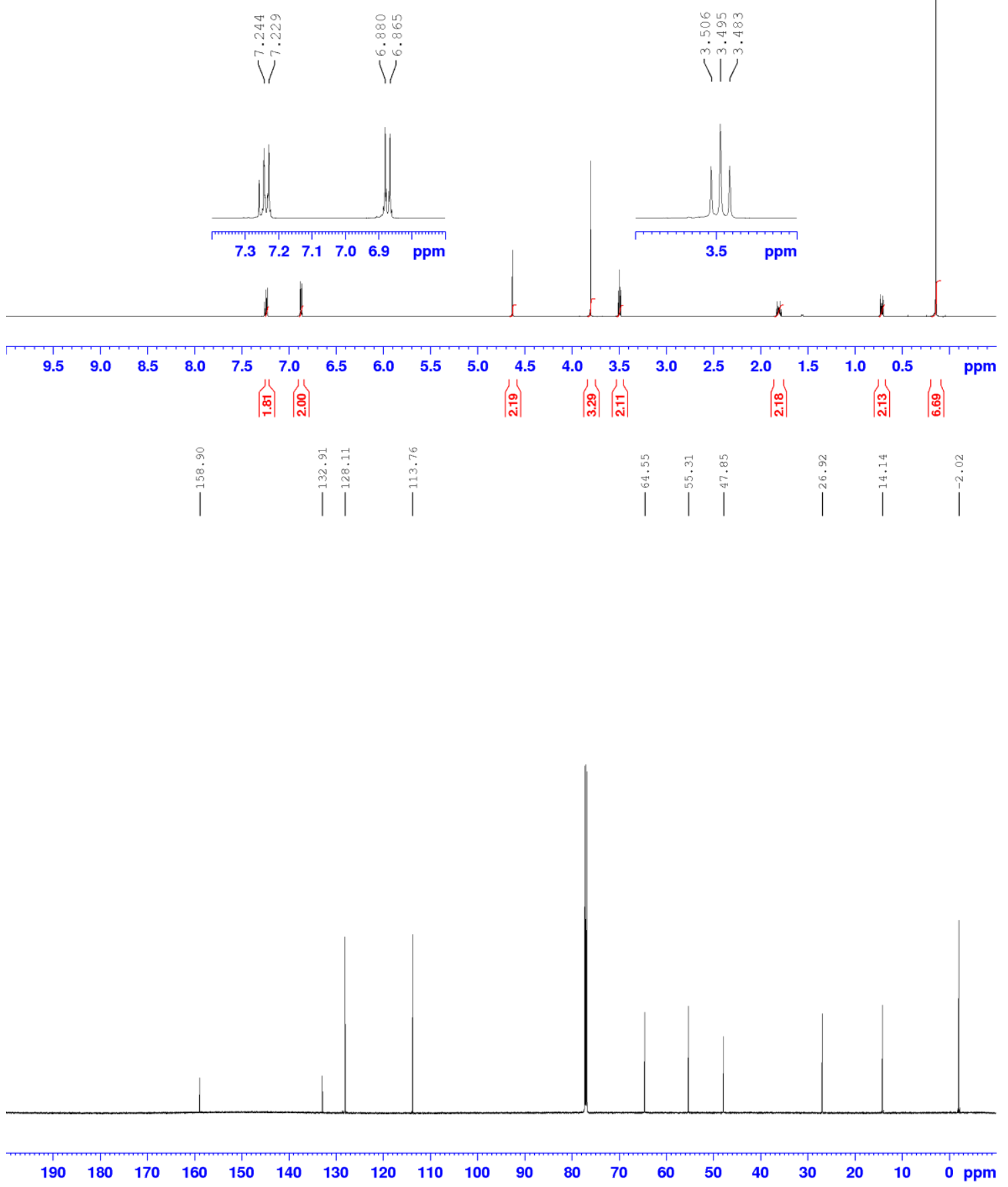

S33 
${ }^{1} \mathrm{H}\left(600 \mathrm{MHz}, \mathrm{CDCl}_{3}\right)$ and ${ }^{13} \mathrm{C}$ NMR Spectra $\left(150 \mathrm{MHz}, \mathrm{CDCl}_{3}\right)$ of $4 \mathrm{i}$
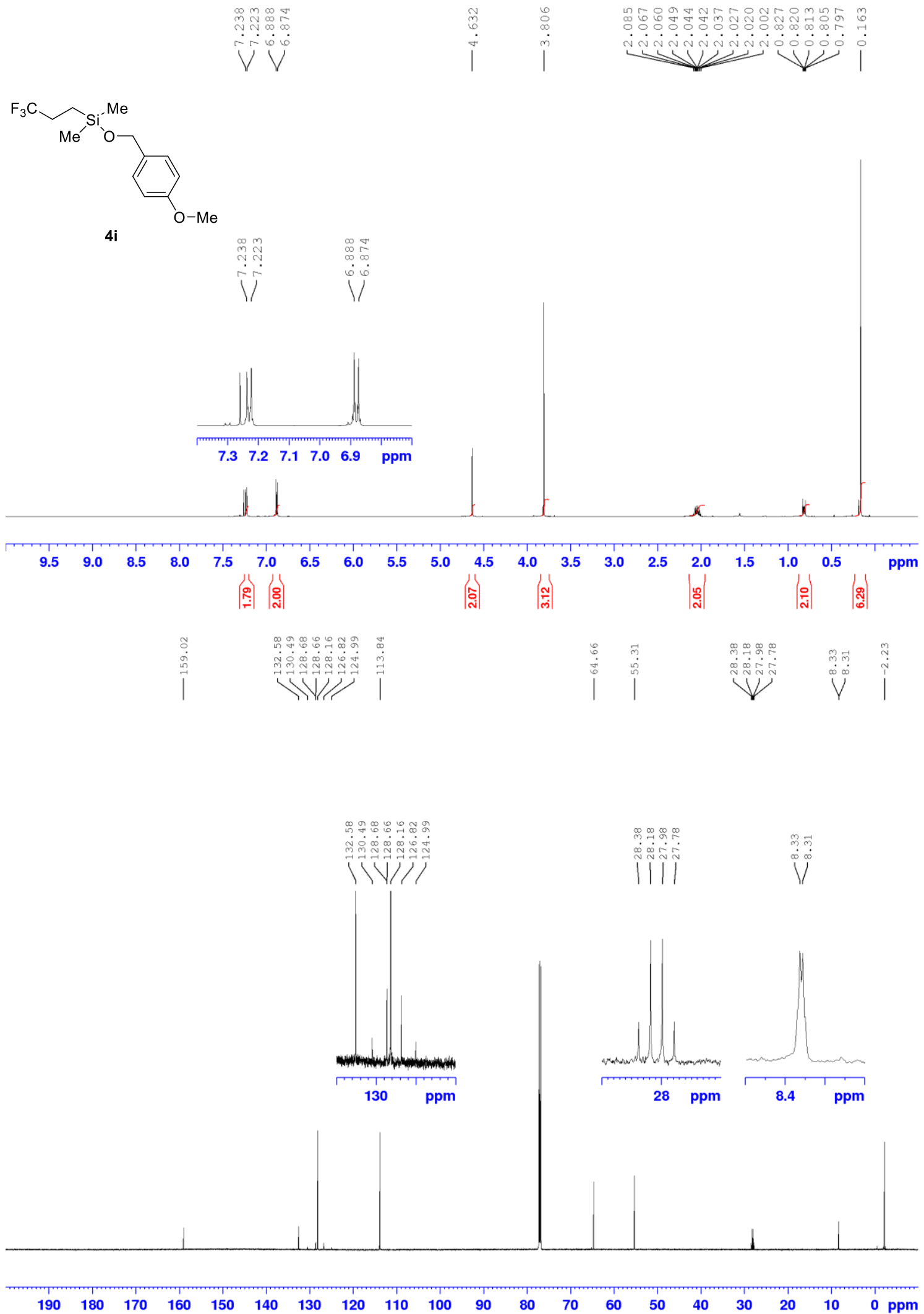
${ }^{1} \mathrm{H}\left(600 \mathrm{MHz}, \mathrm{CDCl}_{3}\right)$ and ${ }^{13} \mathrm{C}$ NMR Spectra (150 MHz, CDCl$)$ of $4 \mathrm{j}$

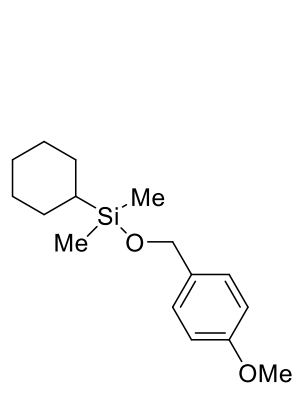

oj
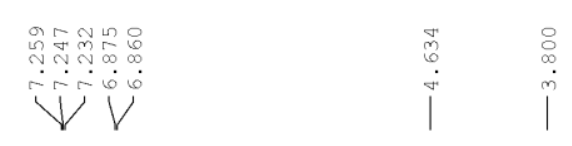
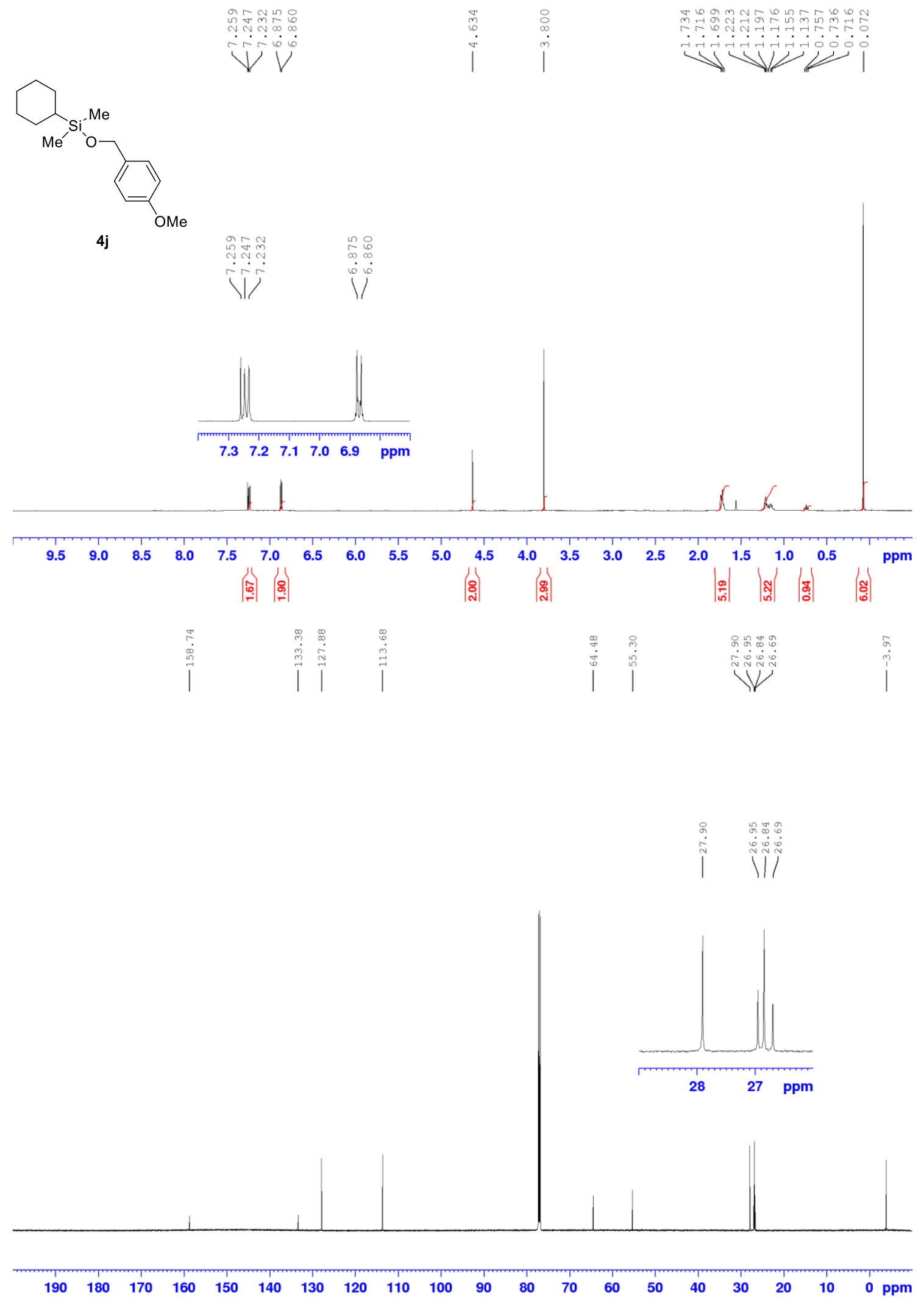

S35 
${ }^{1} \mathrm{H}\left(600 \mathrm{MHz}, \mathrm{CDCl}_{3}\right)$ and ${ }^{13} \mathrm{C}$ NMR Spectra (150 MHz, $\left.\mathrm{CDCl}_{3}\right)$ of $4 \mathrm{k}$
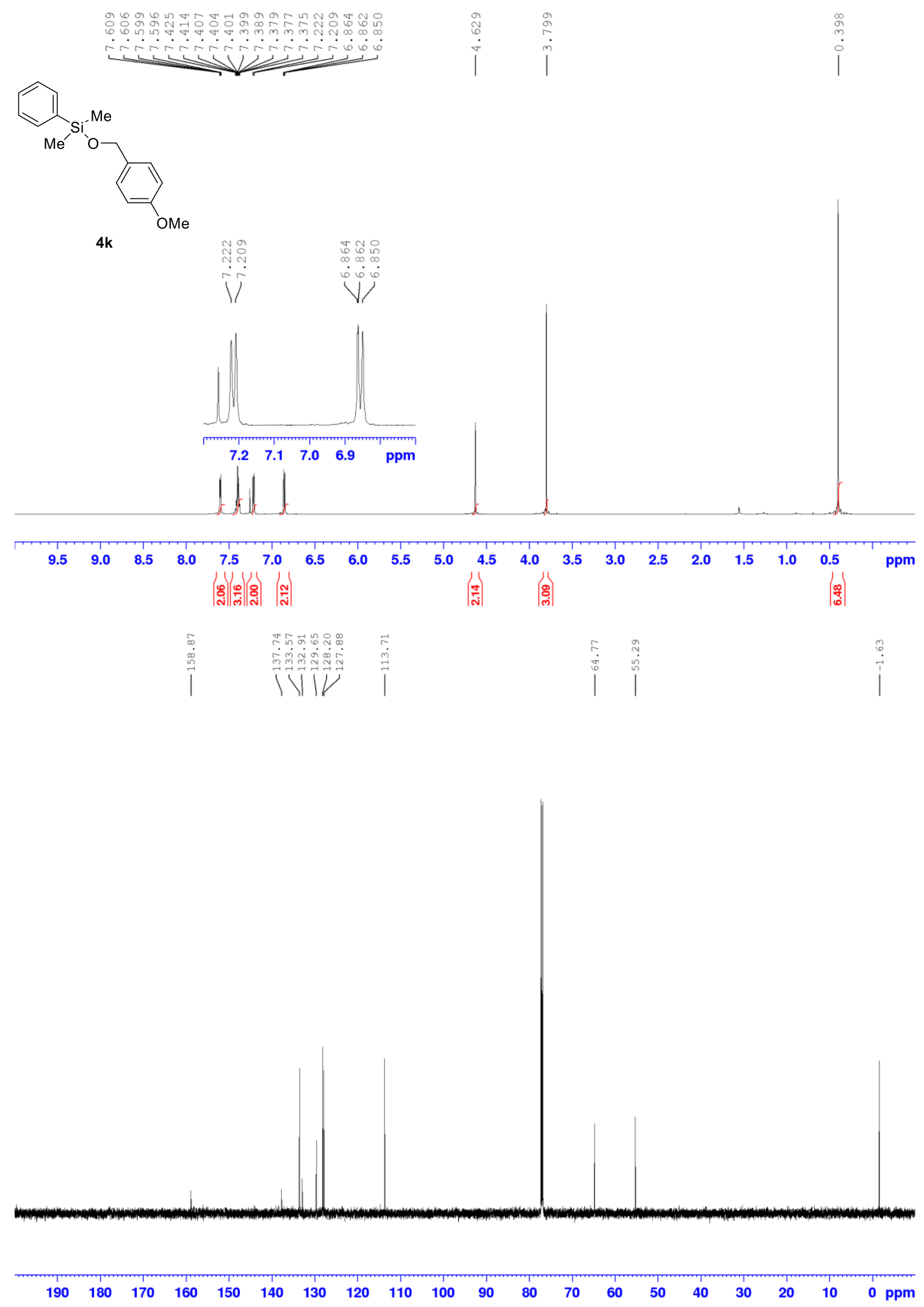
${ }^{1} \mathrm{H}\left(600 \mathrm{MHz}, \mathrm{CDCl}_{3}\right)$ and ${ }^{13} \mathrm{C}$ NMR Spectra (150 $\left.\mathrm{MHz}, \mathrm{CDCl}_{3}\right)$ of 41
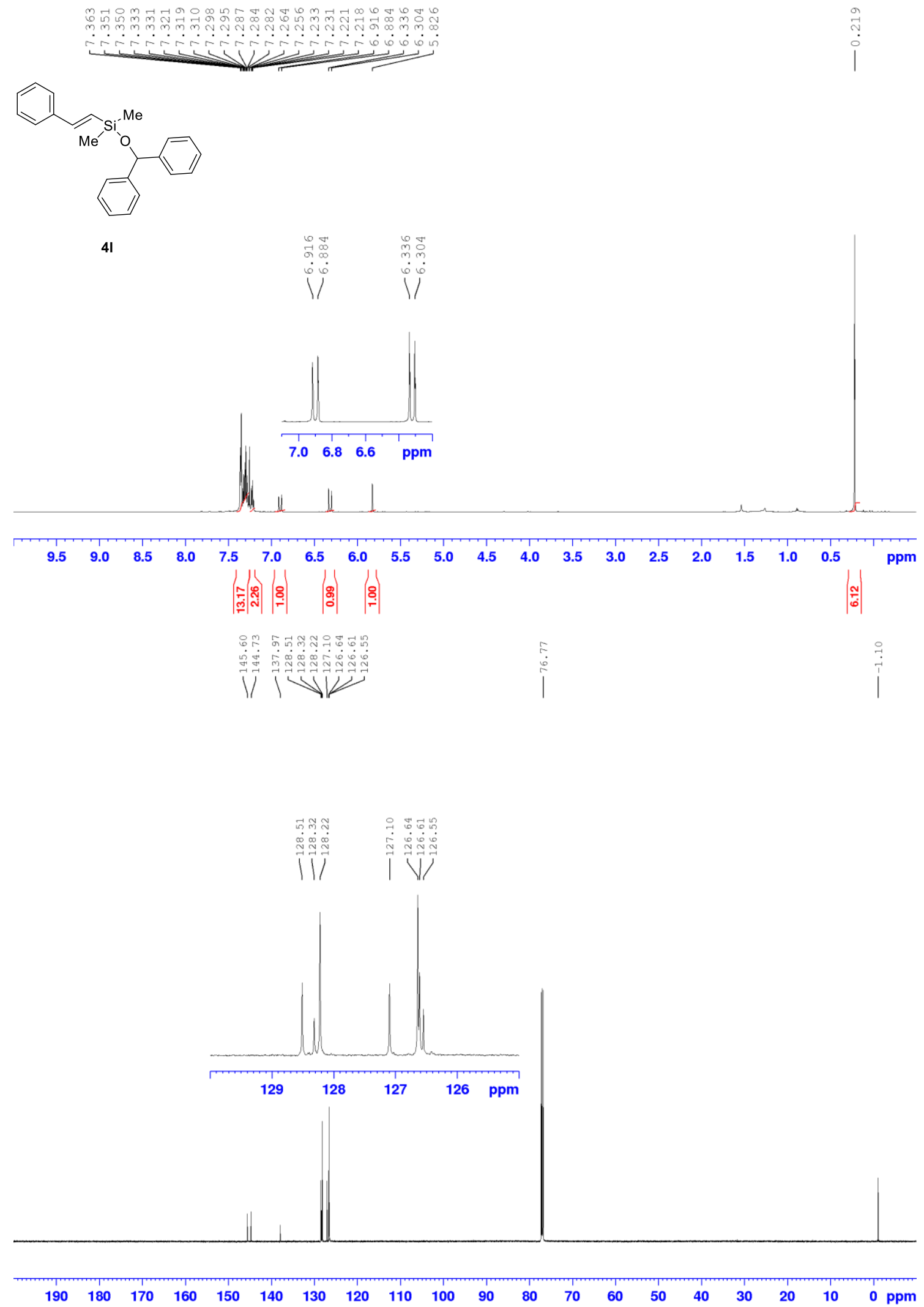
${ }^{1} \mathrm{H}\left(600 \mathrm{MHz}, \mathrm{CDCl}_{3}\right)$ and ${ }^{13} \mathrm{C}$ NMR Spectra (150 MHz, CDCl 3$)$ of $4 \mathrm{~m}$
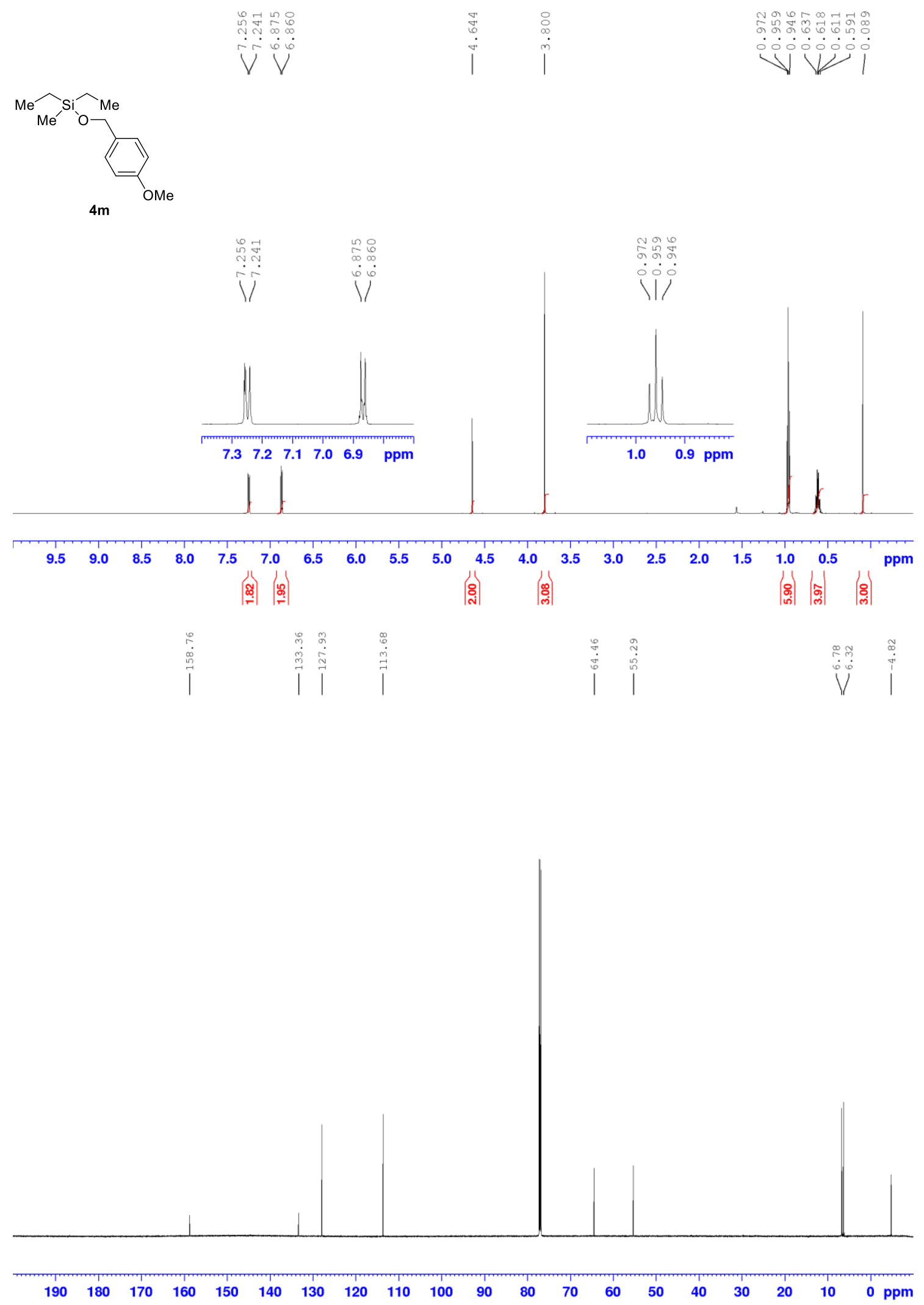
${ }^{1} \mathrm{H}\left(600 \mathrm{MHz}, \mathrm{CDCl}_{3}\right)$ and ${ }^{13} \mathrm{C}$ NMR Spectra (150 MHz, $\left.\mathrm{CDCl}_{3}\right)$ of $4 \mathrm{n}$
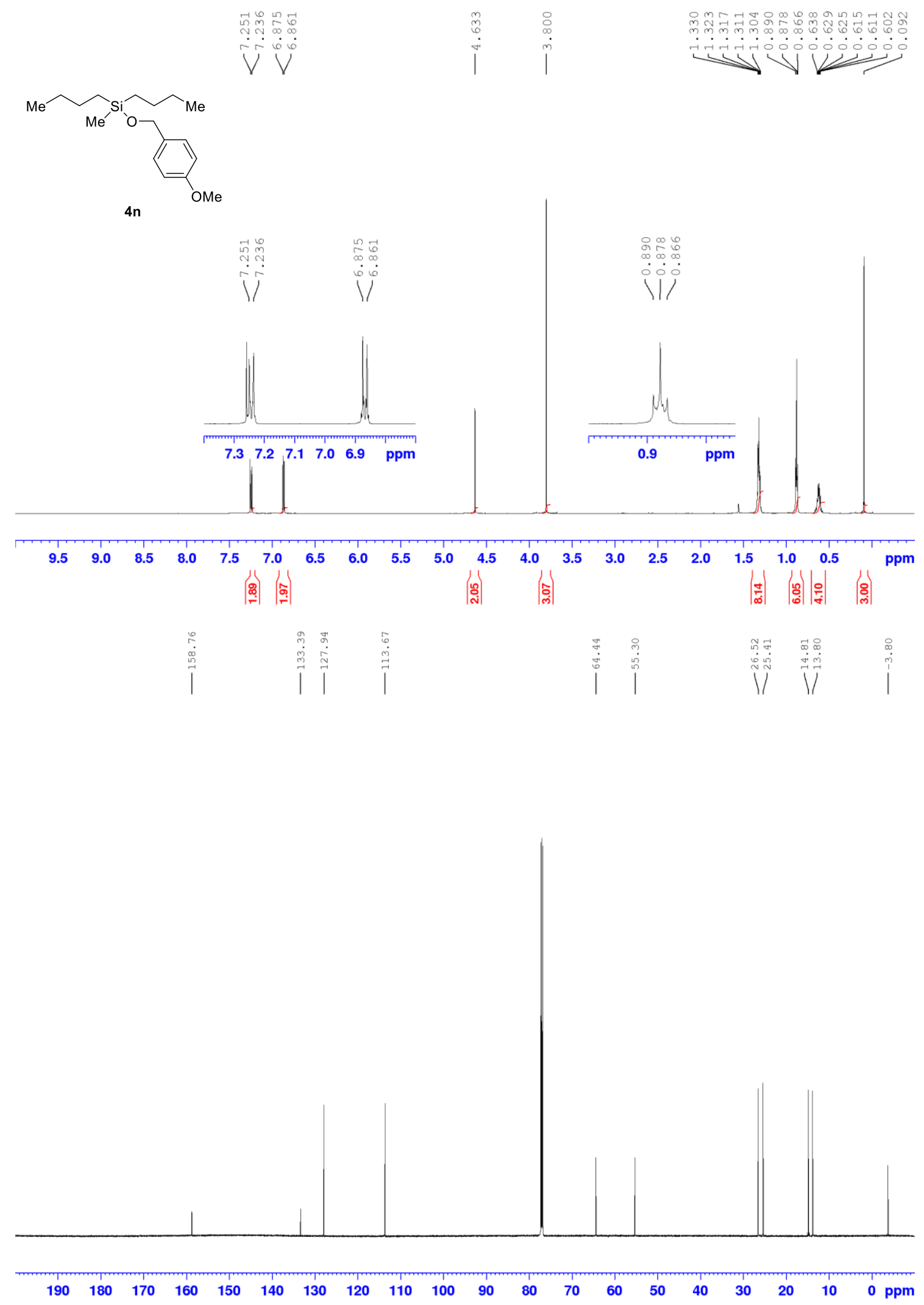
${ }^{1} \mathrm{H}\left(600 \mathrm{MHz}, \mathrm{CDCl}_{3}\right)$ and ${ }^{13} \mathrm{C}$ NMR Spectra (150 MHz, $\left.\mathrm{CDCl}_{3}\right)$ of 40
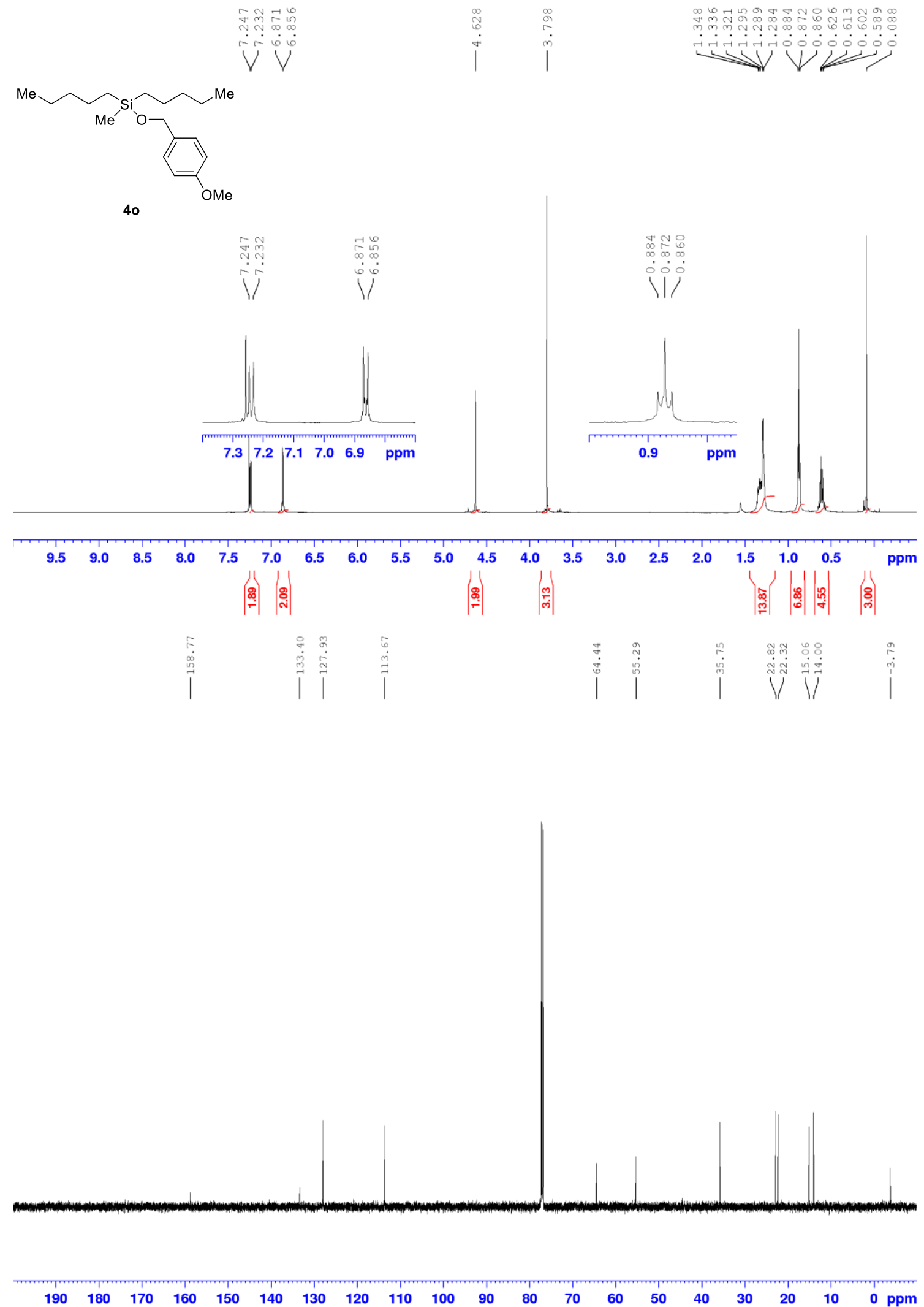
${ }^{1} \mathrm{H}\left(600 \mathrm{MHz}, \mathrm{CDCl}_{3}\right)$ and ${ }^{13} \mathrm{C}$ NMR Spectra (150 MHz, $\left.\mathrm{CDCl}_{3}\right)$ of $4 \mathrm{p}$
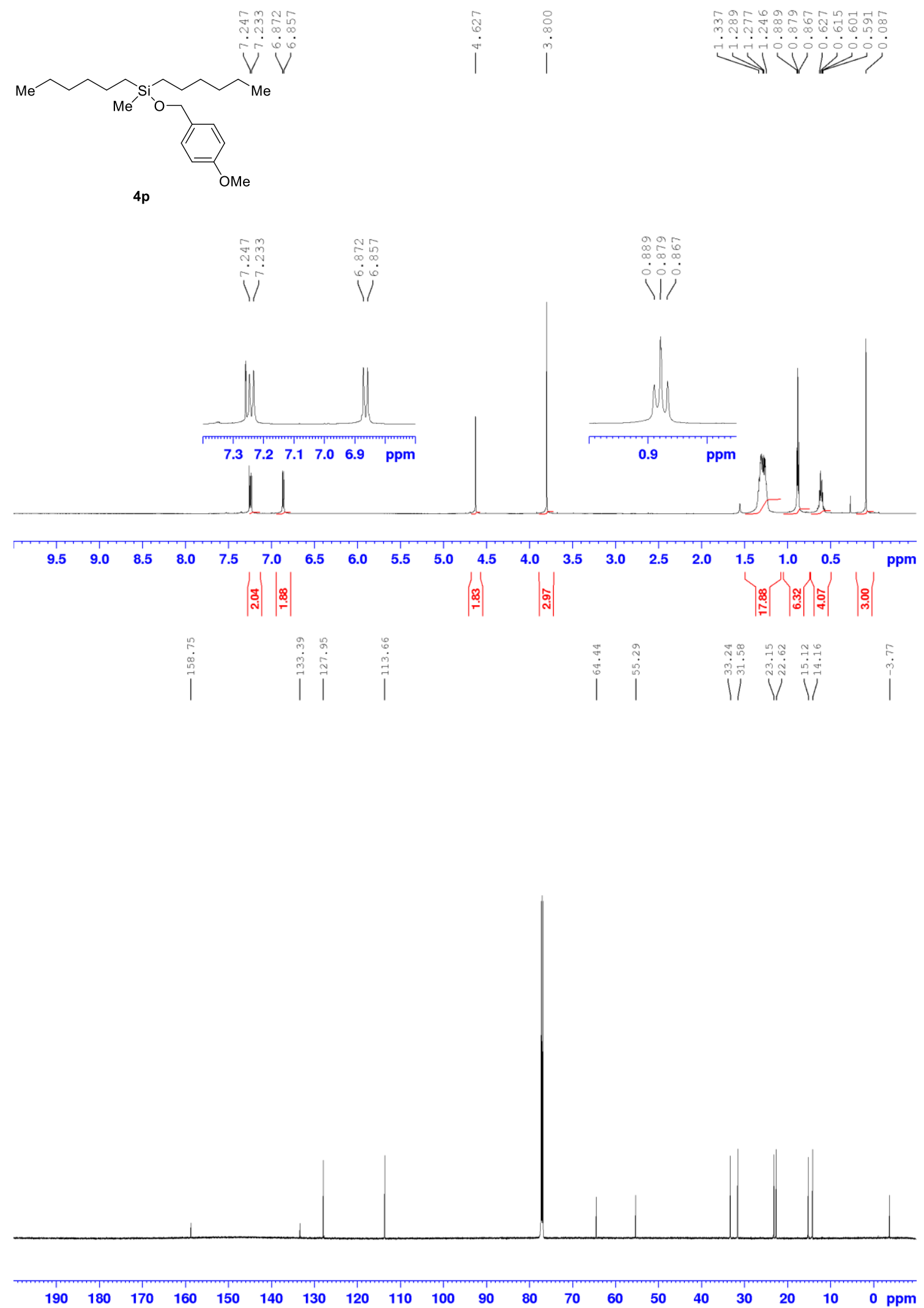
${ }^{1} \mathrm{H}\left(600 \mathrm{MHz}, \mathrm{CDCl}_{3}\right)$ and ${ }^{13} \mathrm{C}$ NMR Spectra (150 MHz, CDCl 3$)$ of $4 \mathrm{q}$

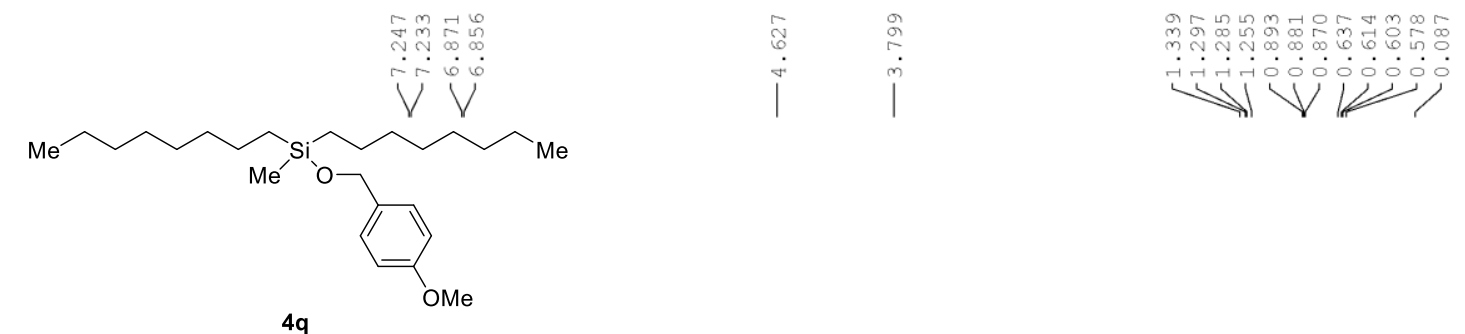

$4 q$
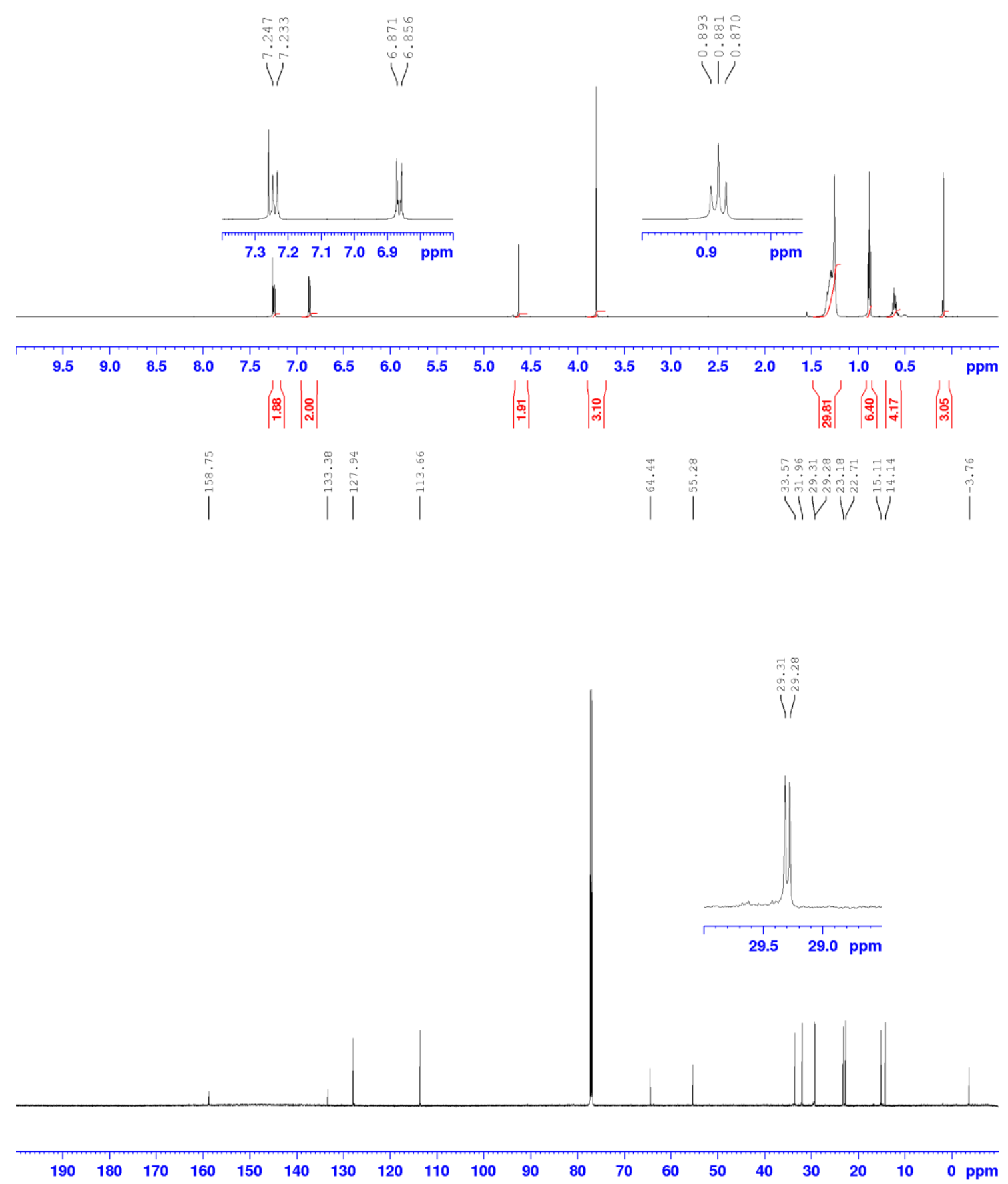
${ }^{1} \mathrm{H}\left(600 \mathrm{MHz}, \mathrm{CDCl}_{3}\right)$ and ${ }^{13} \mathrm{C}$ NMR Spectra (150 MHz, $\left.\mathrm{CDCl}_{3}\right)$ of $4 \mathrm{r}$
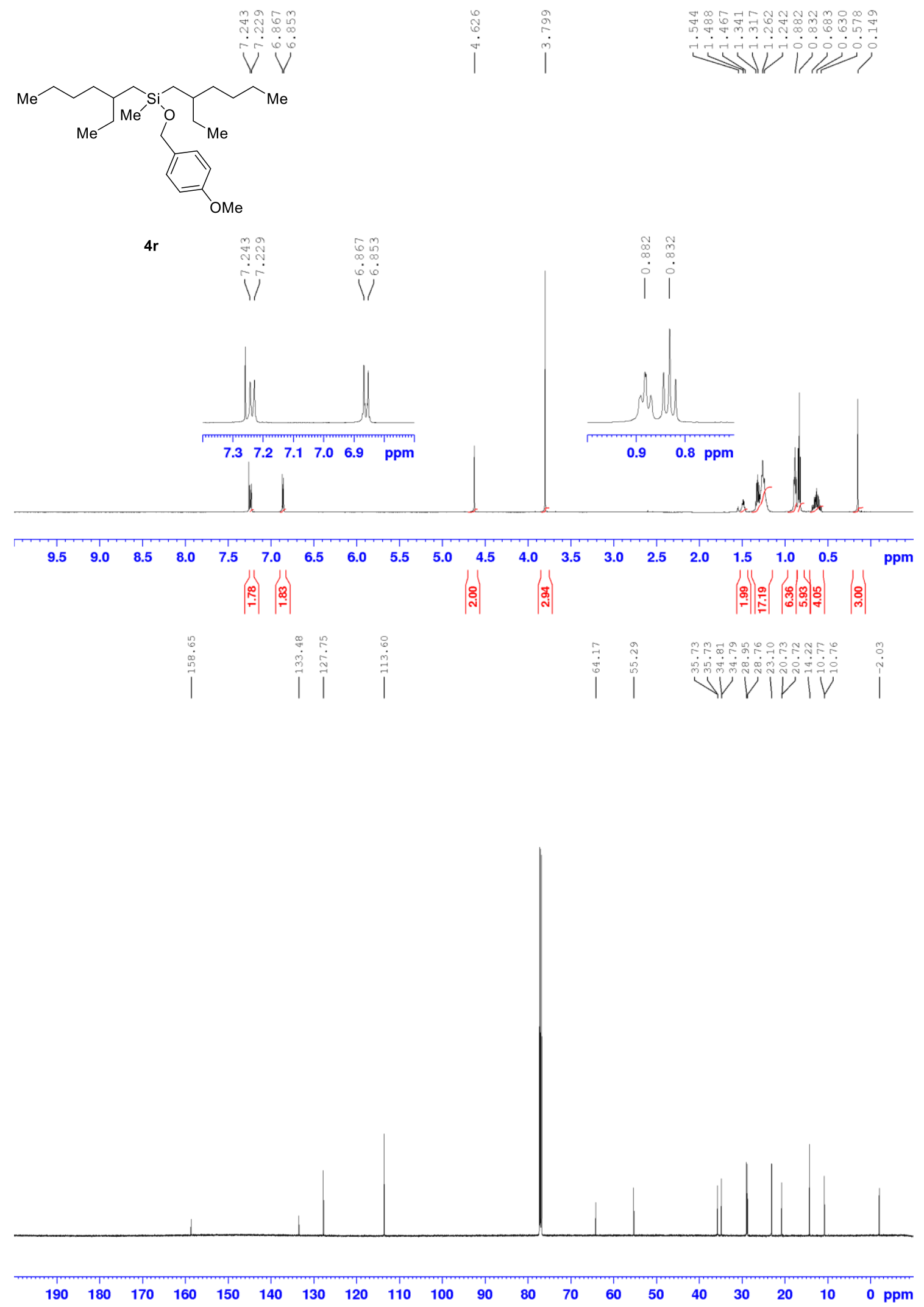
${ }^{1} \mathrm{H}\left(600 \mathrm{MHz}, \mathrm{CDCl}_{3}\right)$ and ${ }^{13} \mathrm{C}$ NMR Spectra (150 MHz, $\left.\mathrm{CDCl}_{3}\right)$ of $4 \mathrm{~s}$
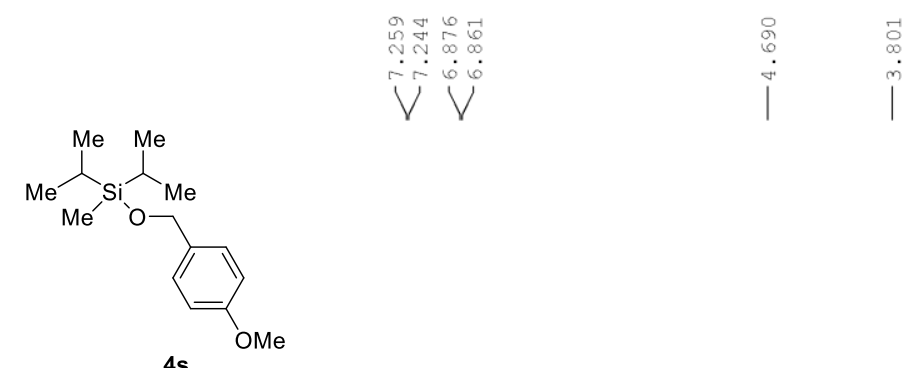

匀证 |
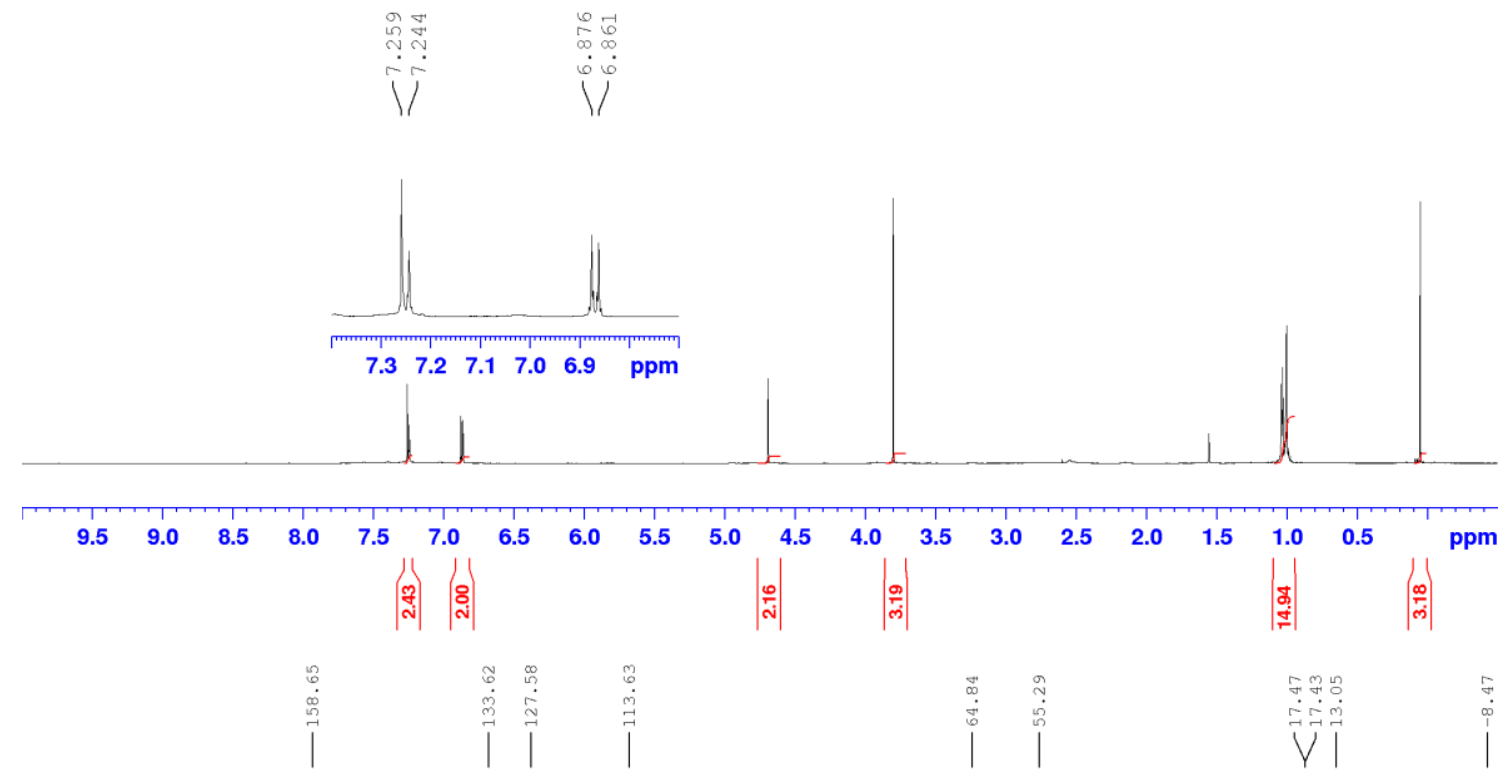

V|

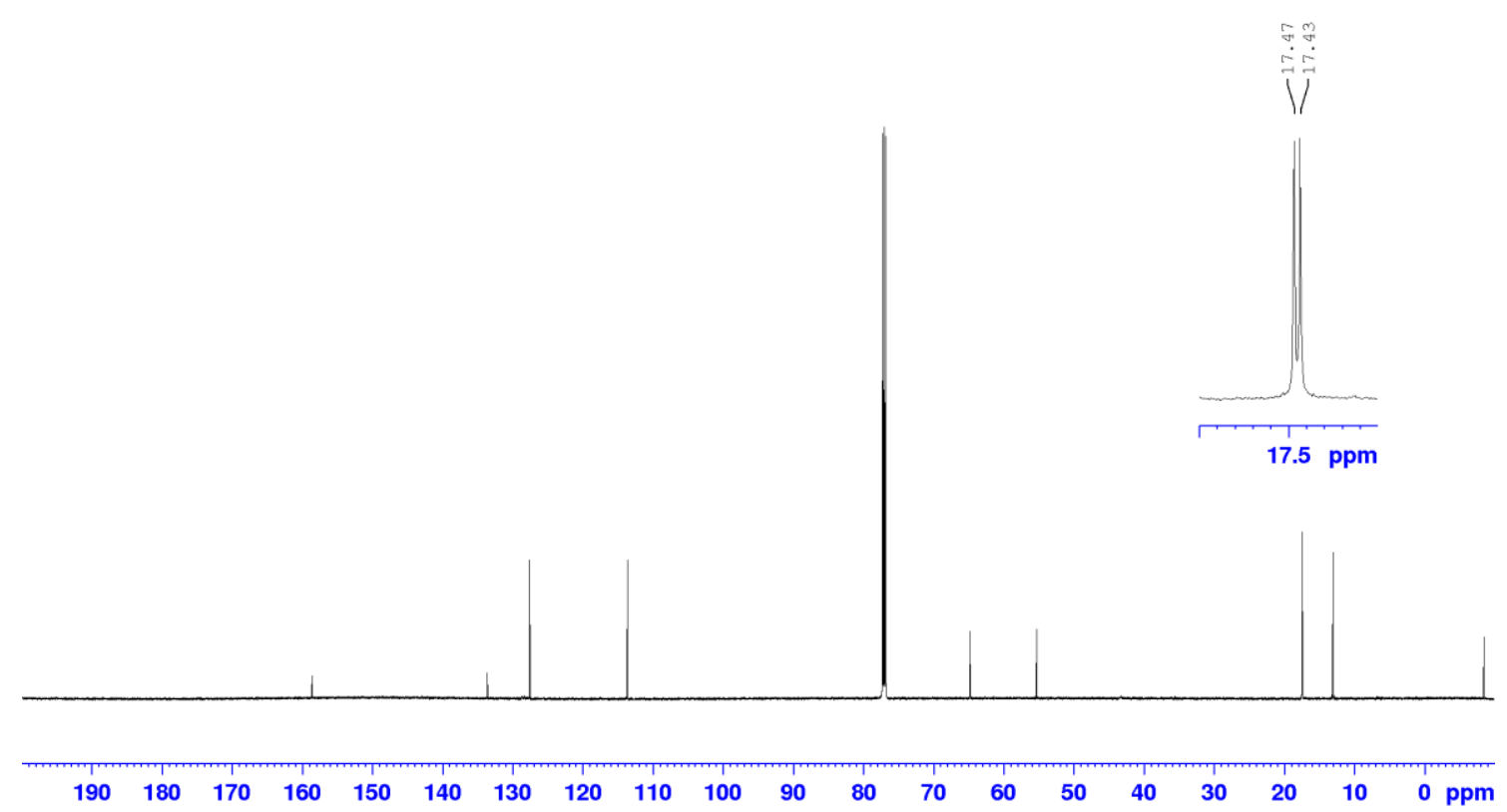


${ }^{1} \mathrm{H}\left(600 \mathrm{MHz}, \mathrm{CDCl}_{3}\right)$ and ${ }^{13} \mathrm{C}$ NMR Spectra (150 MHz, $\left.\mathrm{CDCl}_{3}\right)$ of $4 \mathrm{u}$
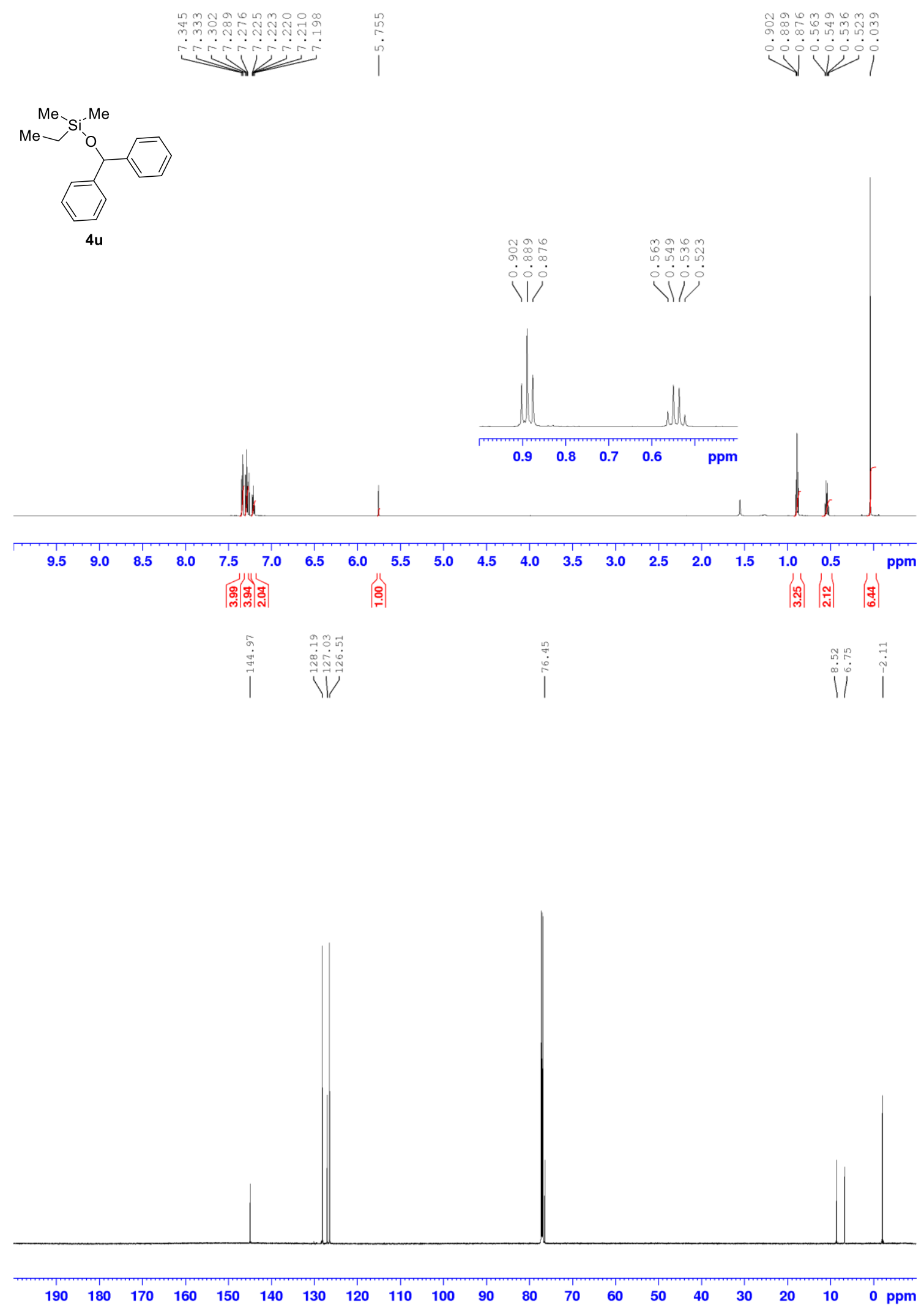
${ }^{1} \mathrm{H}\left(600 \mathrm{MHz}, \mathrm{CDCl}_{3}\right)$ and ${ }^{13} \mathrm{C}$ NMR Spectra (150 $\left.\mathrm{MHz}, \mathrm{CDCl}_{3}\right)$ of $4 \mathrm{v}$

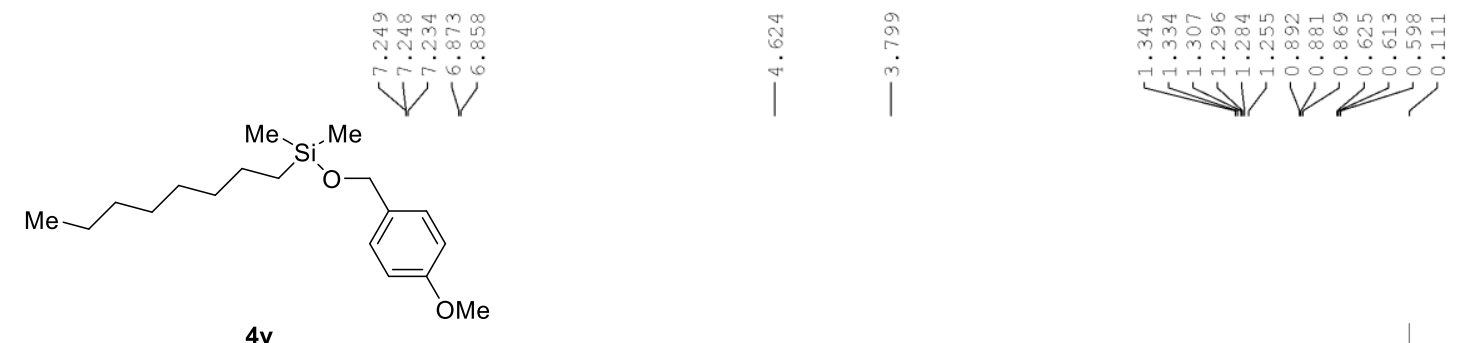

$4 \mathbf{v}$
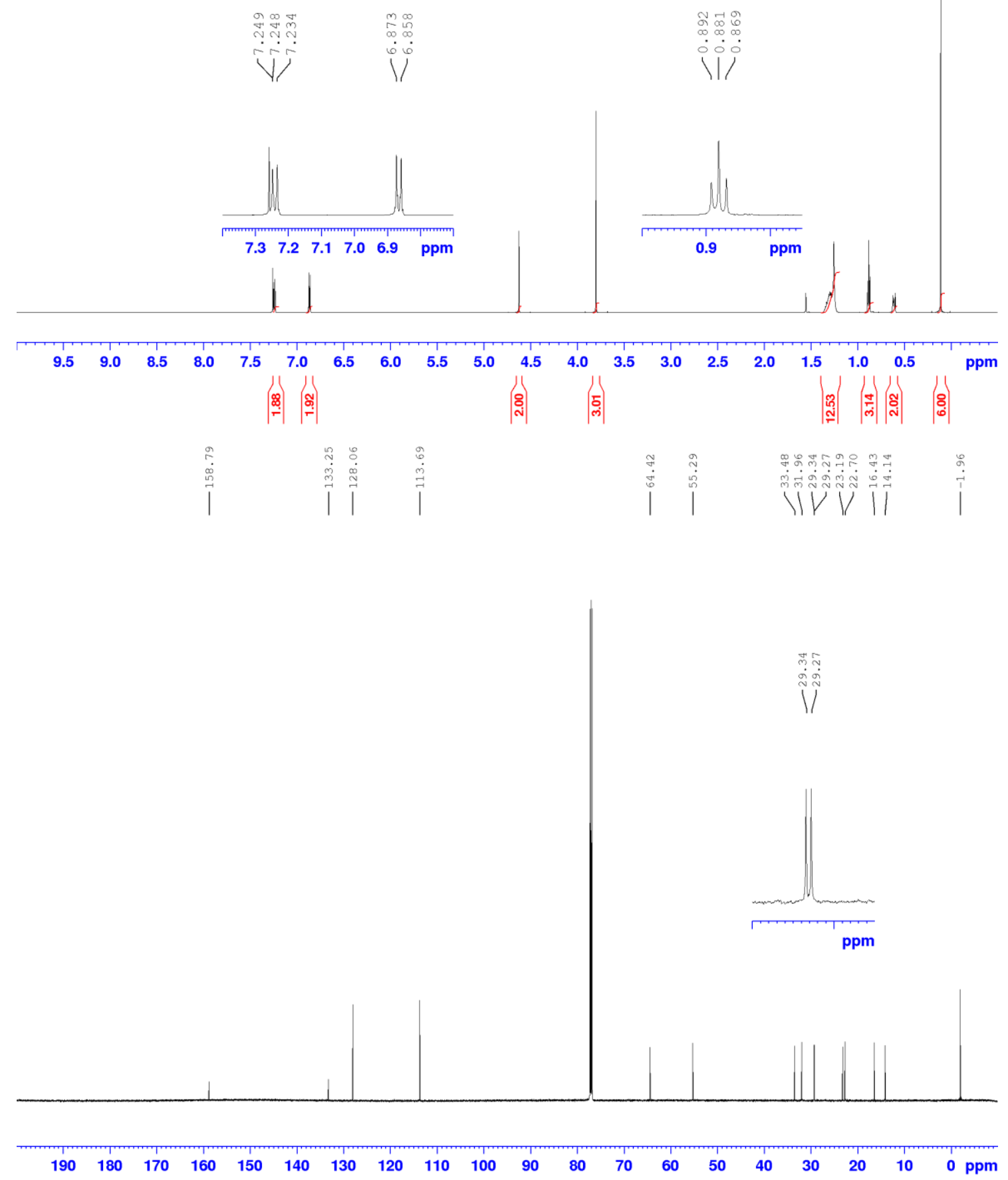
${ }^{1} \mathrm{H}\left(600 \mathrm{MHz}, \mathrm{CDCl}_{3}\right)$ and ${ }^{13} \mathrm{C}$ NMR Spectra (150 $\left.\mathrm{MHz}, \mathrm{CDCl}_{3}\right)$ of $4 \mathrm{w}$
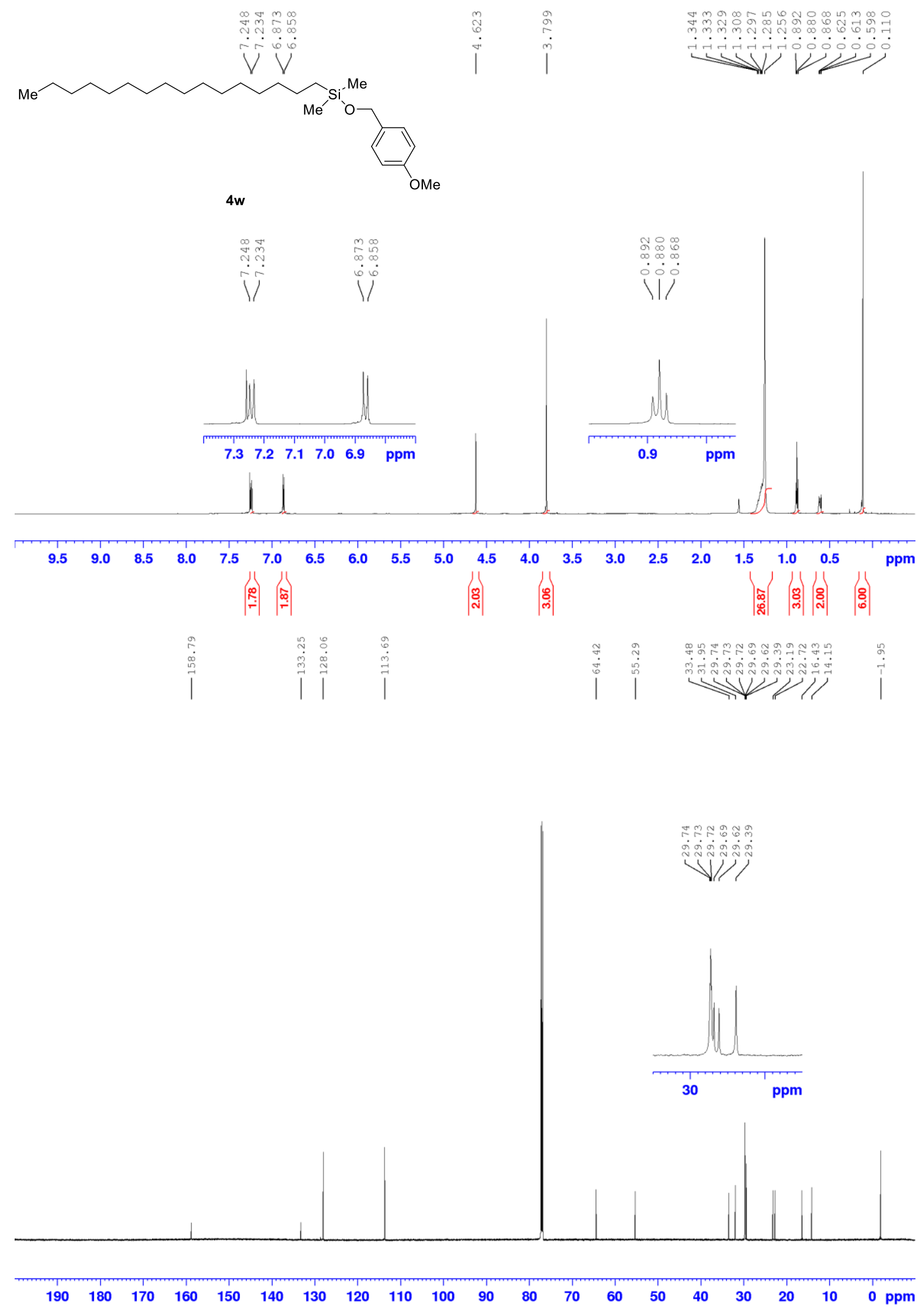
${ }^{1} \mathrm{H}\left(600 \mathrm{MHz}, \mathrm{CDCl}_{3}\right)$ and ${ }^{13} \mathrm{C}$ NMR Spectra (150 MHz, CDCl 3$)$ of $4 \mathrm{x}$
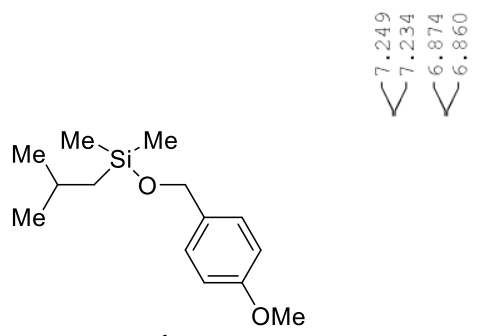

$4 \mathbf{x}$
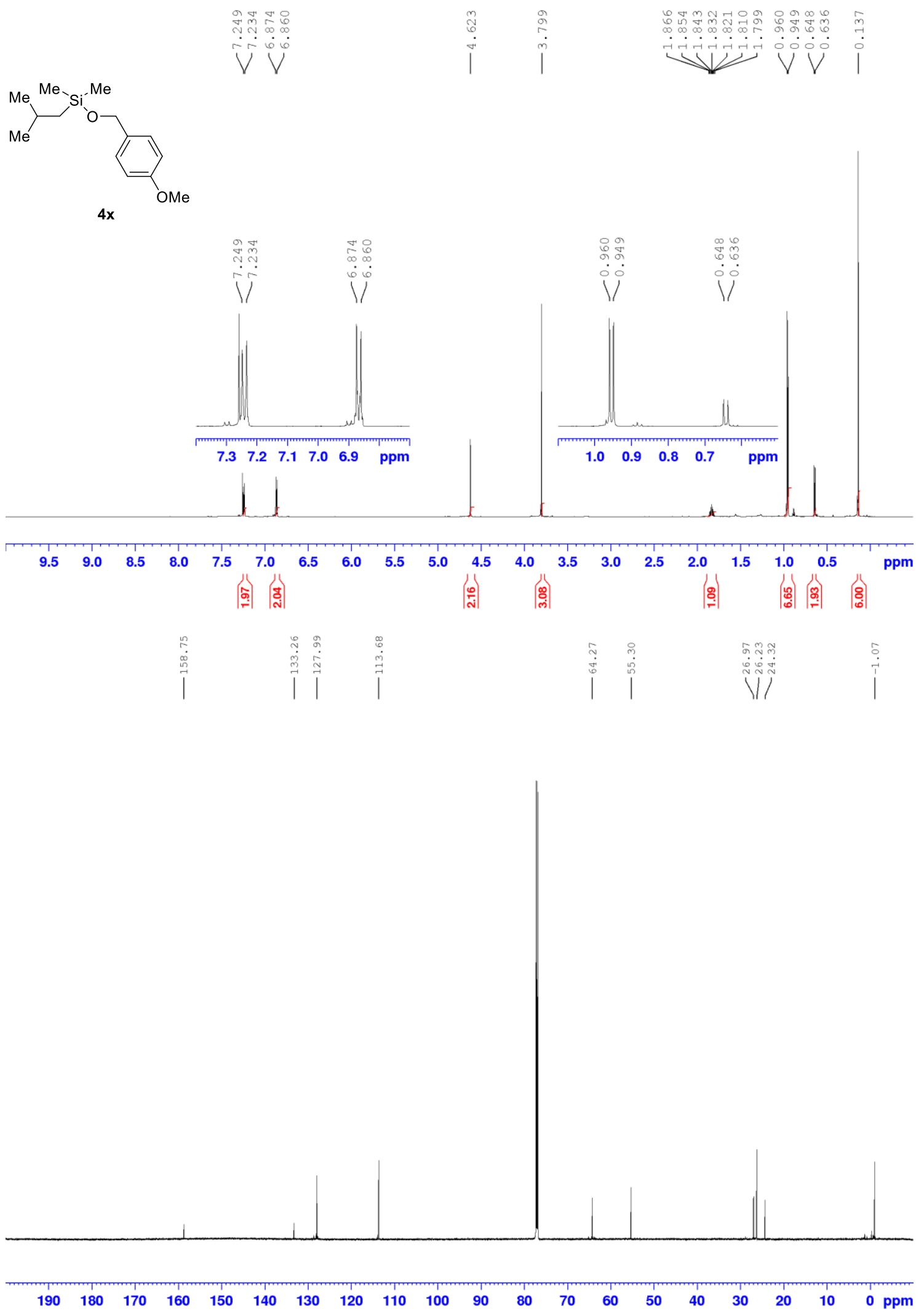

S48 
${ }^{1} \mathrm{H}\left(600 \mathrm{MHz}, \mathrm{CDCl}_{3}\right)$ and ${ }^{13} \mathrm{C}$ NMR Spectra (150 $\left.\mathrm{MHz}, \mathrm{CDCl}_{3}\right)$ of $4 \mathrm{y}$
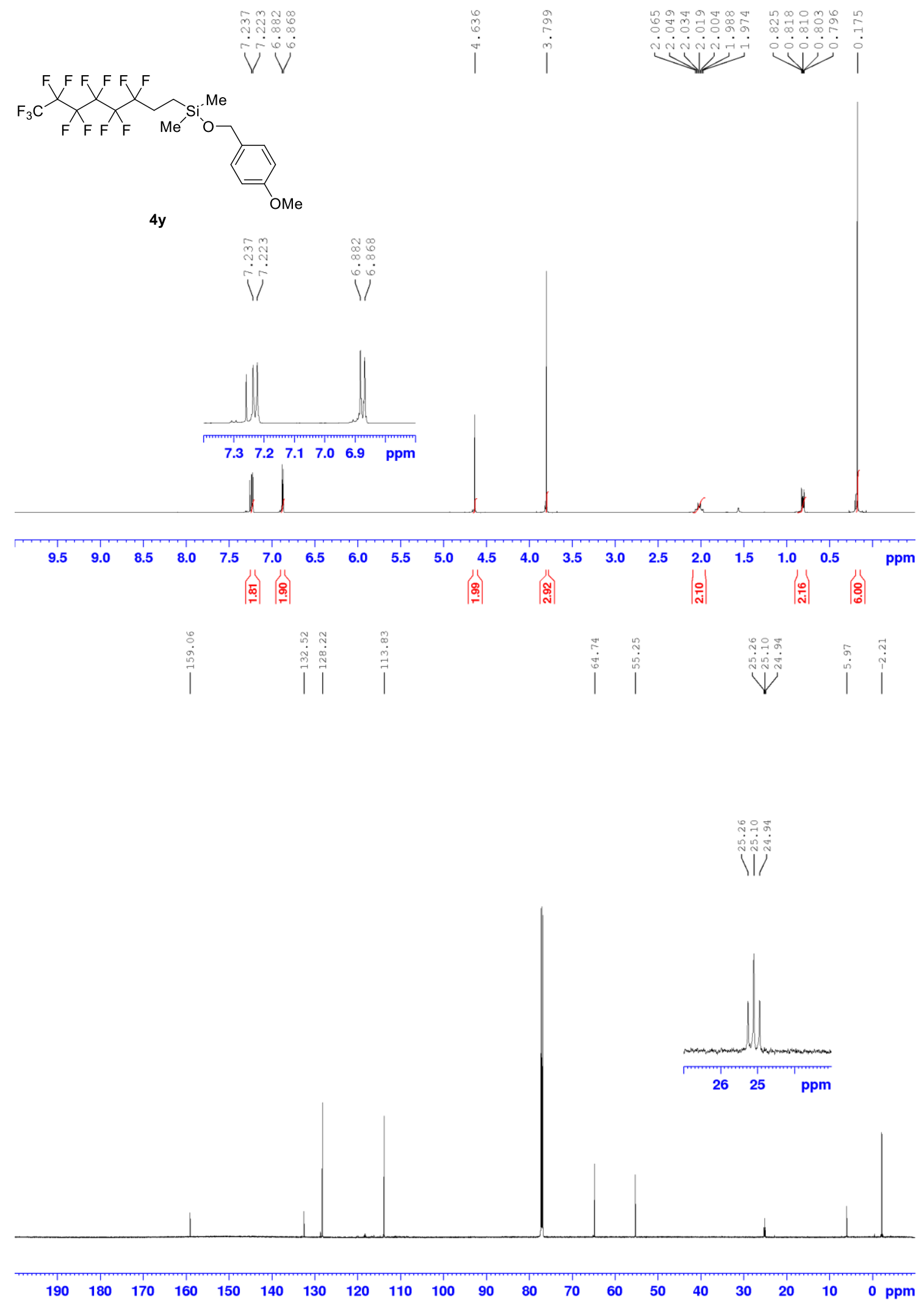
${ }^{1} \mathrm{H}\left(600 \mathrm{MHz}, \mathrm{CDCl}_{3}\right)$ and ${ }^{13} \mathrm{C}$ NMR Spectra (150 MHz, CDCl 3$)$ of $4 \mathrm{z}$
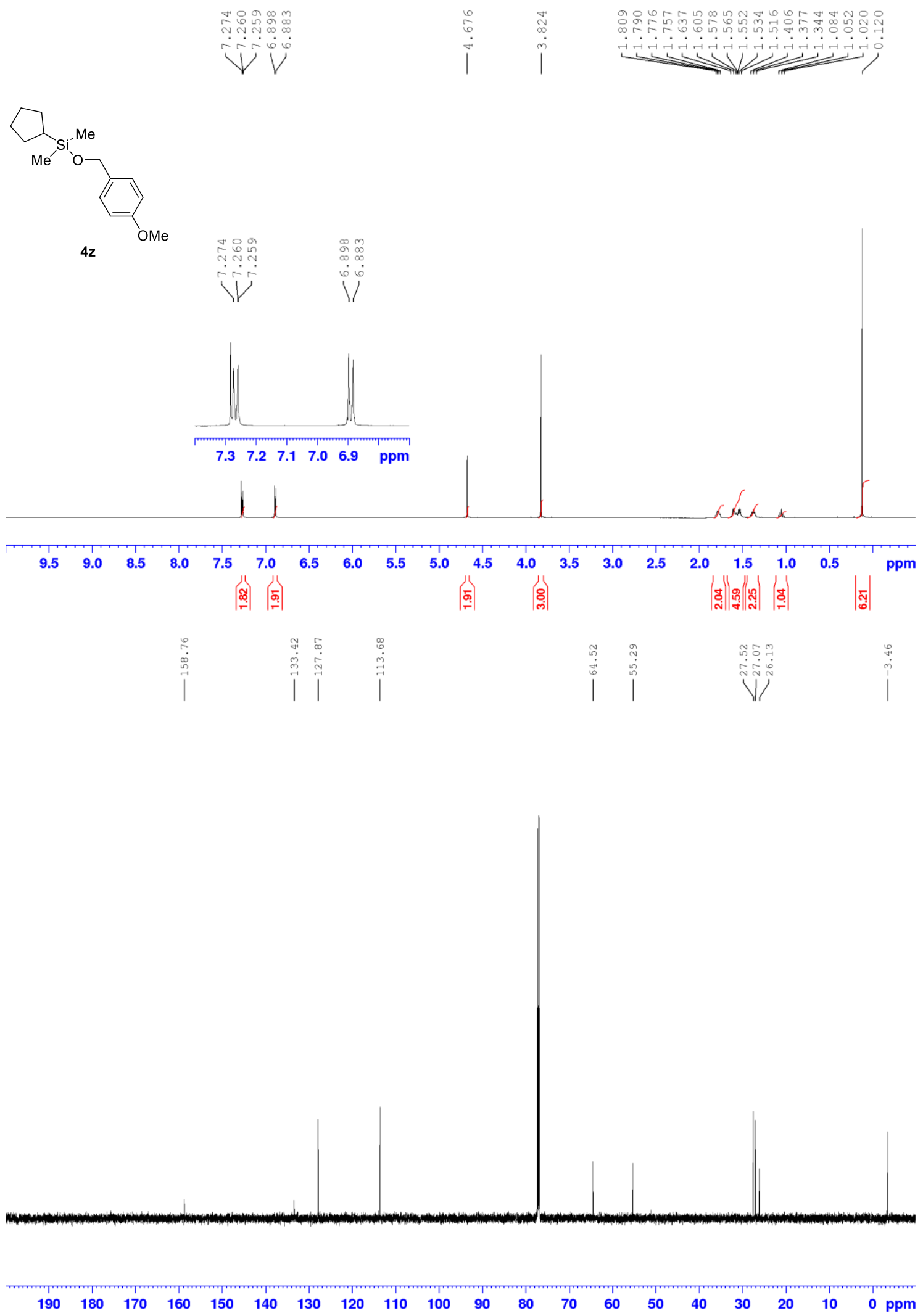
${ }^{1} \mathrm{H}\left(600 \mathrm{MHz}, \mathrm{CDCl}_{3}\right)$ and ${ }^{13} \mathrm{C}$ NMR Spectra $\left(150 \mathrm{MHz}, \mathrm{CDCl}_{3}\right)$ of Aaa
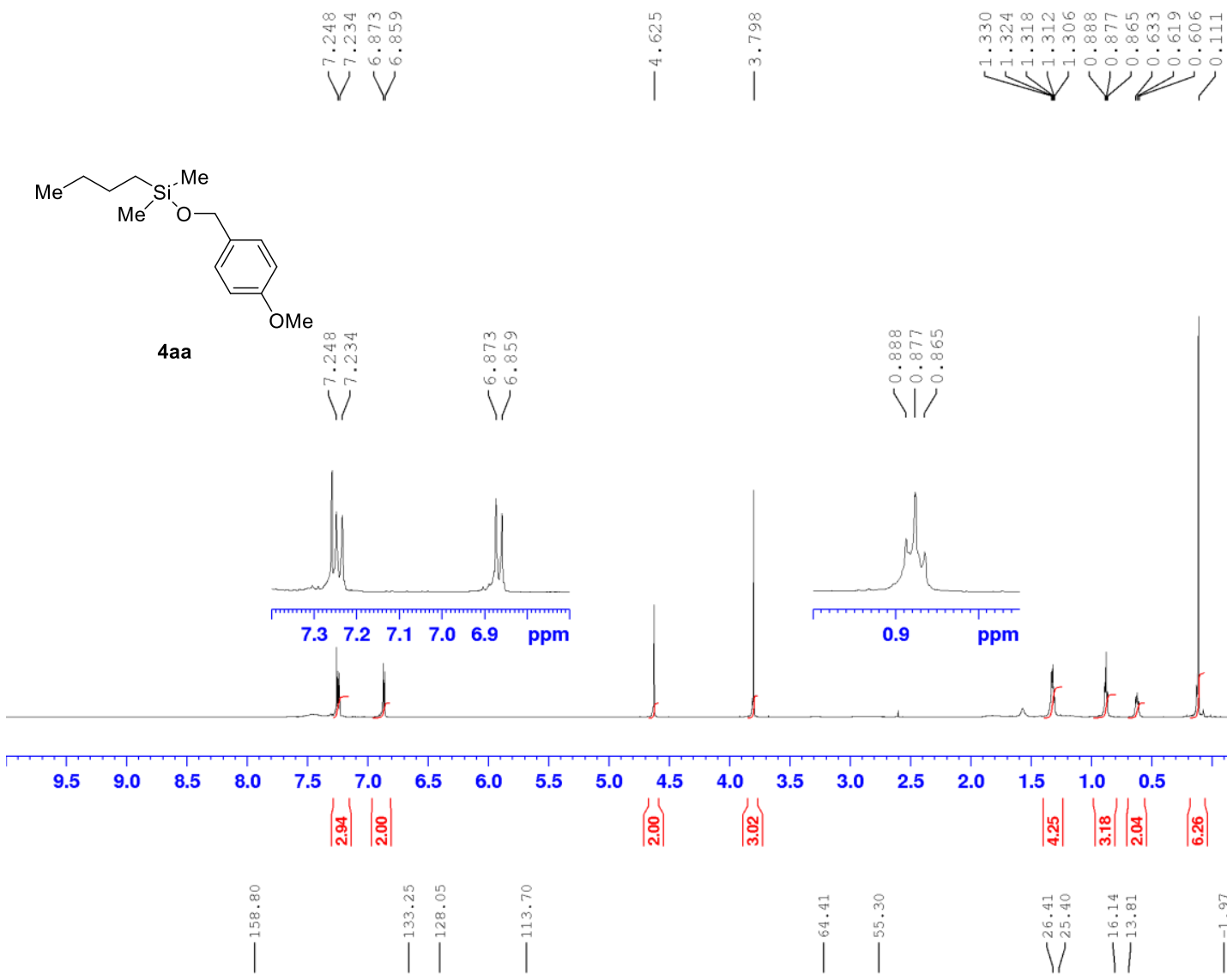

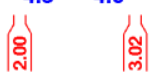
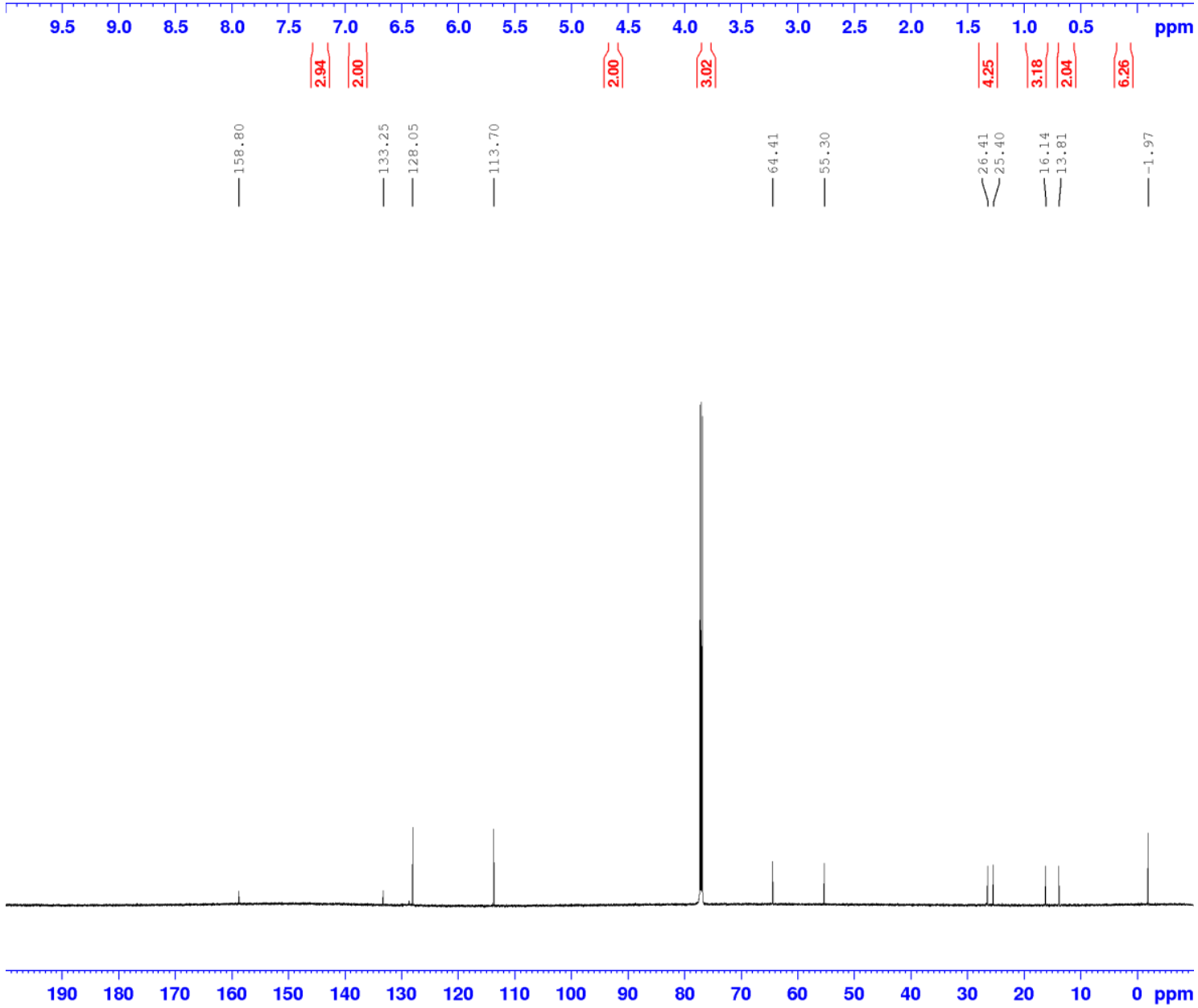

S51 